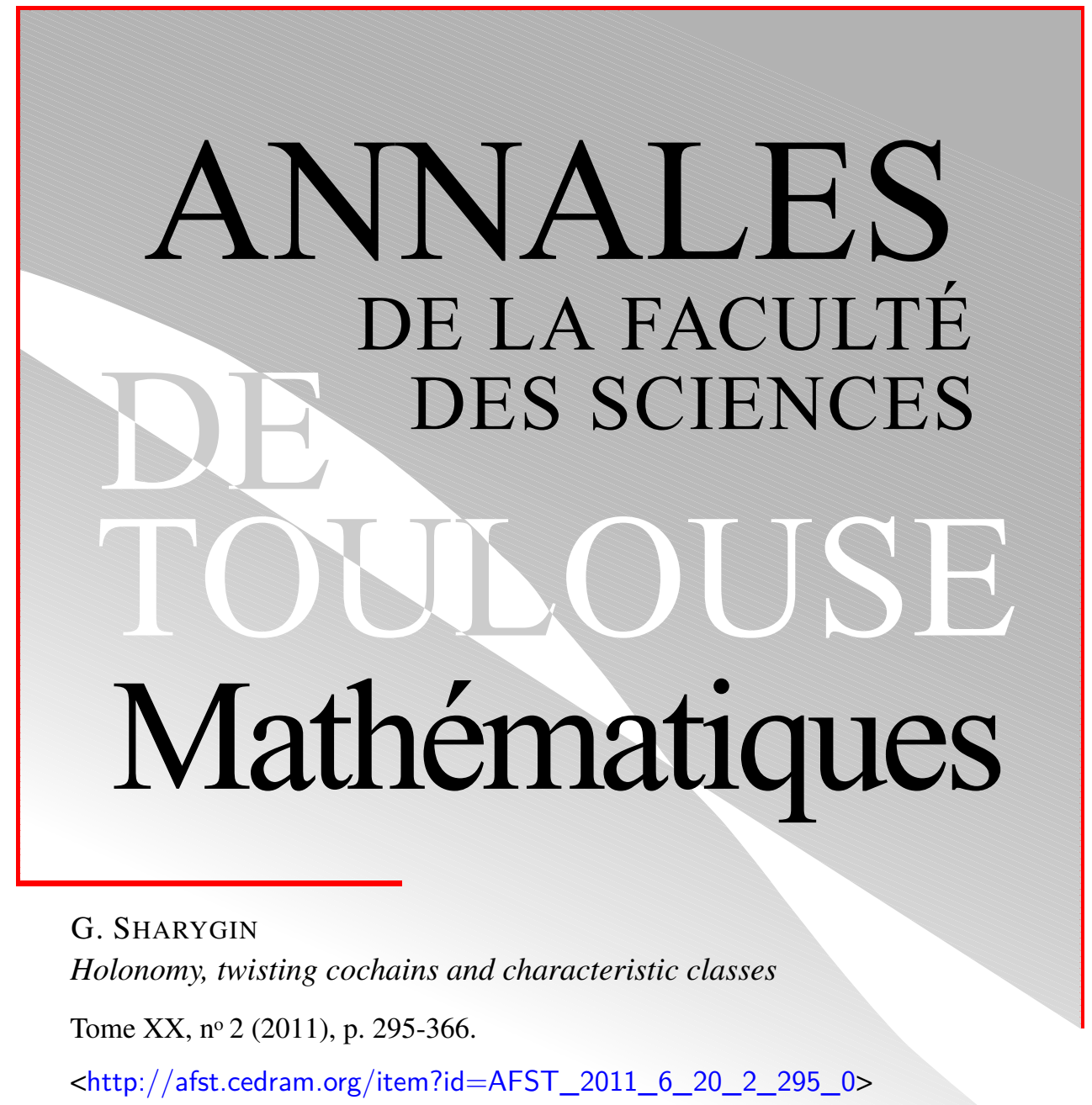

C Université Paul Sabatier, Toulouse, 2011, tous droits réservés.

L'accès aux articles de la revue «Annales de la faculté des sciences de Toulouse Mathématiques » (http://afst.cedram.org/), implique l'accord avec les conditions générales d'utilisation (http://afst.cedram. org/legal/). Toute reproduction en tout ou partie cet article sous quelque forme que ce soit pour tout usage autre que l'utilisation à fin strictement personnelle du copiste est constitutive d'une infraction pénale. Toute copie ou impression de ce fichier doit contenir la présente mention de copyright.

\title{
cedram
}

Article mis en ligne dans le cadre du

Centre de diffusion des revues académiques de mathématiques

http://www.cedram.org/ 


\title{
Holonomy, twisting cochains and characteristic classes
}

\author{
G. Sharygin ${ }^{(1)}$
}

\begin{abstract}
This paper contains a description of various geometric constructions associated with fibre bundles, given in the terms of important algebraic object, the twisting cochain. Our examples include the ChernWeil classes, holonomy representation and the so-called cyclic Chern character of Bismut and others (see [2, 11, 27]), also called the Bismut's class. The latter example is the principal one for us, since we are motivated by the attempt to find an algebraic approach to the Witten's index formula. We also give several examples of twisting cochains associated with a given principal bundle. In particular, our approach allows us to obtain explicit formulas for the Chern classes and for an analogue of the cyclic Chern character in the terms of the glueing functions of the principal bundle. We discuss few modifications of this construction. We hope that this approach can turn fruitful for the investigations of the Witten index formula.
\end{abstract}

RÉSUMÉ. - Nous proposons diverses constructions naturelles que l'on peut associer à un espace fibré. Nous les décrivons à l'aide d'un objet algébrique appelé «cochaîne tordue» («twisting cochaîn»). Nous considérons les classes caractéristiques de Chern-Weil, la représentation d'holonomie et le caractére de Chern cyclique défini par Bismut. Nous cherchons une approche algébrique de la formule de l'index de Witten. En outre, nous donnons quelques constructions explicites de la «cochaîne tordue» associée au fibré principal donné. En particulier, nos méthodes permettent d'obtenir des formules explicites pour les classes de Chern et pour un analogue du caractére de Chern cyclique en fonction du cocycle noncommutatif qui définit ce fibré principal. Nous discutons aussi certaines versions modifiées de cette construction. On espère que ces idées peuvent être utile pour l'étude de formule d'index de Witten.

(*) Reçu le 06/06/2010, accepté le 08/09/2010

(1) Institute of Theoretical and Experimental Physics 25 ul. B. Cheremushkinskaya, Moscow, 117259 Russia. sharygin@itep.ru 


\section{G. Sharygin}

\section{Introduction}

This paper is a result of the author's attempt to give an algebraic description of the well-known results of Witten, Bismut, Getzler, Jones, Petrack and others, which describe the index of Dirac operator on a vector bundle in terms of pairing of two characteristic classes in equivariant homology and cohomology of the free loop space of the base. In effect, the following formula is true:

$$
\operatorname{ind}_{E} D=\left\langle\operatorname{ch}(D), \operatorname{ch}_{E}(\nabla)\right\rangle,
$$

where $D$ is a Dirac operator on the $\operatorname{Spin}_{\mathbb{C}}$ manifold $X$ and $E$ is a vector bundle on $X$. The class $c h(D)$ has degree 0 , it is the so-called Witten's class of $D$ in equivariant (with respect to the natural action of the circle) homology of the free loop space of $X, \mathcal{L} X$. It is given by a formal expression, involving integration over $\mathcal{L} X$. The nature of this object is somewhat mysterious and far from being completely understood. And on the right of the bracket there stands another characteristic class, Bismut's class (or cyclic Chern character), determined by a connection on the bundle $E$. It belongs to the degree 0 equivariant cohomology of $\mathcal{L} X$. In the paper [11] this class was described by a cocycle in the (reduced) cyclic complex of the de Rham algebra of $X$. In the present paper we develop further the ideas of [11] and try to express $c h_{E}(\nabla)$ in the terms of such a fundamental algebraic-topological object as twisting cochain. We have a humble hope that it will be possible in future to apply similar ideas to the left side of this formula and obtain an algebraic description of Witten's class too.

Let us recall that the notion of twisting cochains was first introduced in algebraic topology by E. Brown (see [7] and section 1.3 below). In the cited paper of Brown it is shown that for every principal bundle $P$ over a space $X$ with structure group $G$ (we shall always assume that all three spaces are compact closed manifolds and that $X$ is 1-connected) there exists a twisting cochain on the coalgebra of singular chains on the base with values in the Pontriagin algebra of singular chains on the structure group, such that the corresponding twisted tensor product models the total space of the fibre bundle. Moreover, it can be shown that this correspondence is 1-1 modulo an equivalence relation, similar to the gauge equivalence of flat connections. Thus one can regard twisting cochains as algebraic counterparts of bundles and connections and ask the following questions: "What is the precise relation between twisting cochains and connections? Is it possible to express twisting cochains in terms of connections and vice-versa? In particular, in what way one can express the characteristic classes, in terms of the twisting cochains? In what way one can express holonomy map, in terms of twisting

cochains? In what way one can express Bismut's class, in terms of twisting cochains?" In the present paper we give a partial answer for this questions. 
This answer is three-fold. To begin with, we suggest few explicit constructions for twisting cochains associated with a principal bundle. One should bear in mind, that in the cited paper of Brown, the author works in the homological setting, i.e. differentials in all algebras and coalgebras that he uses decrease degrees by 1 , while in our paper we prefer to deal with the corresponding cohomological analogues. As a result there's a slight change of definitions and few other minor corrections. In particular, we cannot use Brown's theorem directly, instead of this we give an explicit construction of twisting cochain in the situation we consider (see proposition 2.1 and also section 2.3). We regard the notion of twisting cochain as a convenient algebraic model for principal bundles and connections. This approach enables us to extend the ideas and methods of differential geometry to the wider domain of differential graded algebras and coalgebras, that are not necessarily algebras of de Rham forms a manifold. One can work with twisting cochains pretty much like one works with connections (flat or non-flat).

The composition of the paper is as follows: after we have reminded the reader in chapter 1 few principal definitions and results from the fields we need, in chapter 2 we address the first part of the question above: "Is it possible to use a twisting cochain like one uses connections to find characteristic classes of the principal bundle?" The answer is positive. Namely, after we have constructed a twisting cochain, (proposition 2.1), we express Chern classes in terms of this cochain. In effect, it is well-known that twisting cochains induce characteristic maps from the cobar resolution of the coalgebra to the algebra in which they take values (see $[20,21,22])$. One can show that the image of this map consists of characteristic classes of the bundle $P$. We use this simple idea and the explicit formula for the twisting cochain to find explicit formulae for the characteristic classes of $P$ expressed in the terms of the glueing functions (i.e. of the corresponding noncommutative Cech cocycle with values in the structure group). This discussion is carried forward in section 2.2, where we give explicit formulae for the first two Chern classes (see equation (2.11) for instance). Further details of this construction can be found in paper [23]. Besides this in section 2.4 we describe a general construction that allows one to obtain the genuine twisitng cochains with values in the commutative DG algebras, modeling the base, e.g. de Rham algebra of the base.

Secondly, to deal similarly with the holonomy of a connection (we discuss it in chapter 3), we regard it as a map from the loop space of the base into the structure group of the bundle. This approach is in a spirit very close to the Kan's theorem (see [15]): there's a 1-1 correspondence between principal bundles over a base and representations of Kan's group of the base (i.e. of the group, homotopy equivalent to the $H$-space $\Omega X$ of based loops on the base). 
There are many models of this group, and many algebraic constructions that can replace its singular cochains. One of the most convenient of them is the Chen's iterated integral construction that gives a collection of differential forms on $\Omega X$. This construction gives a homomorphism of algebras from bar-resolution of the de Rham algebra of a manifold $X$ (the multiplication in $B\left(\Omega_{D R}(X)\right)$ is given by shuffle products) into the algebra of differential forms on the loop space of $X$. One can show (see [8]) that this map induces an isomorphism in cohomology if $X$ is 1-connected. This approach to the loop spaces can be generalized to free loop space of a manifold: it turns out that the iterated integral map can be extended to the map from Hochschild complex of $\Omega_{D R}(X)$ into the de Rham forms on the free loop space of $X$ (see [11] and [14]), which induces an isomorphism in cohomology under the same condition of 1-connectedness of $X$. Moreover, in the cited papers it is also proved that the equivariant cohomology of free loop space with respect to the circle action on it (by translations of the argument) is isomorphic to the cyclic homology of the algebra $\Omega_{D R}(X)$. Thus it is natural to use the bar-complex, Hochschild and cyclic complexes as models for the loop spaces. It is in one of these complexes, where our map will take values.

Here we should make a remark, concerning the terminology we use: we regard the loop space rather as a topological group. Thus the holonomy of a connection becomes for us a homomorphism of groups. This justifies the name of homological monodromy map that we use to describe the inverse image homomorphism, induced by the holonomy of a connection and its purely algebraic analogs that we construct in this paper. This map is introduced in the section 3.2, formula (3.13). We discuss its properties there and in sections 3.3 and 3.4 .

Another goal we pursue is to use twisting cochains to construct a map from an algebraic model of the gauge bundle associated with the given principal one, to the Hochschild complex of the base. Here we call "the gauge bundle of a principal bundle $P$ with structure group $G$ " the space $\hat{P}=P \times_{A d} G$, where $A d$ denotes the adjoint action of group on itself. The complex that plays the rôle of algebraic model of the gauge bundle is a "bitwisted" tensor product $K \hat{\otimes}_{\phi} A$ of algebra and coalgebra, see definition 3.2 and proposition 3.3. We show that there's a map $\tilde{\hat{\phi}}$ from $K \hat{\otimes}_{\phi} A$ into the (reduced) Hochschild complex of $A$. Moreover, if the algebra $A$ modeling $X$ is commutative, then this map intertwines the comultiplication. On the other hand, the homological monodromy map of section 3.3 has similar domain and range. One can ask, if it is in any sense equivalent to $\tilde{\hat{\phi}}$. To answer this question, one has to modify it a little bit so that it become a map from the gauge bundle to the free loop space of the base. We discuss it 
briefly in section 3.4, however, as we do not need the case of general structure group, we only sketch the general case and consider in greater detail the frame bundle $P$ of a vector bundle $E$ of rank $n$ in section 4.1. In this case one can embed $E$ into a trivial rank $N$ bundle $(N \geqslant n)$ and consider the corresponding projector $p$. One obtains a globally defined "Grassmanian" connection on the bundle $E$, the connection form in this case is a global $N \times N$ matrix-valued 1-form on $X$. One can use this form similarly to the trivial case, considered before (section 3.3) and obtain a map from the differential forms on $\hat{P}$ to differential forms on the free loop space of $X$, or rather to the reduced Hochschild complex of $\Omega_{D R}(X)$. We shall call the latter map "Getzler-Jones-Petrack's" map: it is a homomorphism of the Hopf algebras over $\Omega_{D R}(X)$. Now we can prove one of the main results of the paper

THEOREM 4.3. - Under the identifications we made, the Jones-Getzler map $\tilde{\phi}_{E}$ is chain homotopic to the map $\tilde{\hat{\phi}}$.

This result is directly related with our treatment of Bismut's class: we interpret Getzler, Jones and Petrack construction in such a way that $\operatorname{ch}(\nabla)$ becomes an image of a suitable modification of Getzler-Jones-Petrack's map applied to an element in the de Rham algebra of $\hat{P}$. The modification of the Getzler-Jones-Petrack's map is necessary because Bismut's class takes value in equivariant cohomology of $\mathcal{L} X$. We call this modification "the equivariantization". It is discussed at length in section 4.3, see propositions 4.5 and 4.6. It turns out that it is possible to modify the map $\tilde{\hat{\phi}}$ in a similar way, so that its values on cocentral elements in $\Omega_{D R}(G L(n))$ are closed in the corresponding cyclic complex. Unfortunately one cannot prove directly that these elements are equivalent to Bismut's class, because they depend on the choice of twisting cochain, just like Bismut's class depends on the choice of connection. However, if the twisting cochain is determined by Grassmanian connection, then it is evident that our construction gives the same class. In a general case one can expect that it would be possible to use the reasoning similar to the one used in the end of section 3.3 to prove its independence on the choice of the twisting cochain inside the given equivalence class of twisting cochains (see also [27]).

\section{Acknowledgements}

The author would like to express his deepest gratitude to Anton Gerasimov, whose attention and numerous discussions at the early stages of this project helped the author a great deal. I would also like to express my gratitude to all the institutions where I payed visits while working on this 
project, especially to IMPAN, Warsaw, where I took part in the programme "Noncommutative Geometry and Quantum groups," contract No MKTDCT-2004-509794. While working on this project I was supported by the grants NSh-3035.2008.2 and RFFI 09-01-00239a.

\section{Preliminaries and notations}

This section contains a brief synopsis of few classical definitions, notations and results used in the paper. This section is subdivided into five parts, dealing with homological algebra, homotopy of algebras and coalgebras, twisting cochains, simplicial sets and Lie groups and connections respectively. The conventions we mention here are used throughout the text.

\subsection{Homological Algebra}

The principal reference for this paragraph is [16]. All the algebras and coalgebras that we consider are over a fixed characteristic 0 field $\mathrm{lk}(\mathbb{C}$ or $\mathbb{R}$ are usual examples) unless otherwise stated. For a graded lk-module $M=\left\{M^{i}\right\}_{i \geqslant 1}$, its suspension $s M$ (or $M[1]$ ) is the graded module, such that $(s M)^{i}=M^{i+1}$ (the opposite operation will be denoted by $s^{-1} M$ or $M[-1])$. For a homogeneous element $m \in M,|m|$ will denote its degree. The tensor products of graded objects are taken in graded sense, unless otherwise stated.

Differential graded $\mathrm{kk}$-module (or DG module for short) is $\left(M, d_{M}\right), M$ is graded module and $d_{M}: M^{i} \rightarrow M^{i+1}$. We shall omit the subscript $M$ in $d_{M}$, where it will cause no ambiguity. For two DG modules $\left(M, d_{M}\right)$ and $\left(M^{\prime}, d_{M^{\prime}}\right)$ their tensor product is the DG module $\left(M \otimes M^{\prime}, d_{M \otimes M^{\prime}}\right)$ with differential $d_{M \otimes M^{\prime}}=d_{M} \otimes \mathrm{id}_{M^{\prime}}+\mathrm{id}_{M} \otimes d_{M^{\prime}}$. Dually, the corresponding DG module of maps is $\left(\operatorname{Hom}\left(M, M^{\prime}\right), d_{M, M^{\prime}}\right)$ where the differential is $d_{M, M^{\prime}}(f)=d_{M^{\prime}} \circ f-(-1)^{|f|} f \circ d_{M}$.

For an abstract differential graded algebra (or just DG algebra) $A$, its differential is $d_{A}: A^{i} \rightarrow A^{i+1}$, and the product by $m_{A}$ (the subscript $A$ will usually be omitted, as well as the map $m_{A}$ ). For a right (resp. left) module $M$ (resp. $N) m_{M, A}$ (resp. $m_{A, N}$ ) will denote the corresponding $A$-actions. Dually for a differential graded (or $D G$ ) coalgebra $K$, its differential will be $d_{K}: K^{i} \rightarrow K^{i+1}$ and the coproduct $\Delta_{K} ; \Delta_{K}^{n}$ will denote the iterated coproduct $\Delta_{K}^{1}=\Delta_{K}, \Delta_{K}^{2}=\left(\Delta_{K} \otimes \operatorname{id}_{K}\right) \circ \Delta_{K}, \Delta_{K}^{3}=\left(\Delta_{K} \otimes \operatorname{id}_{K}^{2}\right) \circ \Delta_{K}^{2}$, etc. We shall also often use the Sweedler's notation for coproduct: $\Delta_{K}(x)=$ $\sum_{(x)} x^{(1)} \otimes x^{(2)}$ and similarly for iterations of $\Delta_{K}$ (summation sign will often be omitted). In case of the right (resp. left) $K$-comodule $R$ (resp. $L$ ), 
we shall denote the coaction by $r \mapsto r^{(0)} \otimes r^{(1)}$ (resp. $\left.l \mapsto l^{(-1)} \otimes l^{(0)}\right)$, the superscript 0 corresponds to the elements of comodule.

A DG algebra $(A, d)$ is called augmented, if it is equipped with a homomorphism $\tilde{\eta}$ to the 1-dimensional algebra $\tilde{\eta}: A \rightarrow \mathrm{kk}$, called augmentation. For an augmented algebra $\bar{A}=\operatorname{ker} \tilde{\eta} \cong A / \mathrm{kk} \cdot 1$. For a left and right DG $A$-modules $M$ and $N$ respectively we define the two-sided bar-resolution $B(M, A, N)$ (resp. reduced two-sided bar-resolution $\bar{B}(M, A, N)$ ) by

$$
B(M, A, N)=\bigoplus_{k \geqslant 0} M \otimes(s A)^{\otimes k} \otimes N, \quad \bar{B}(M, A, N)=\bigoplus_{k \geqslant 0} M \otimes(s \bar{A})^{\otimes k} \otimes N
$$

with generic elements $m\left[a_{1}|\ldots| a_{k}\right] n$ of degree $|m|+|n|+\sum_{i=1}^{k}\left|s a_{i}\right|$. We shall denote the subspaces of $k+2$-tensors by $B_{k}(M, A, N)$ and $\bar{B}_{k}(M, A, N)$ respectively. The differential in bar-resolution is defined as $d=d_{0}+d_{1}$, where $d_{0}: B_{k}(M, A, N) \rightarrow B_{k}(M, A, N), \quad d_{1}: B_{k}(M, A, N) \rightarrow B_{k-1}(M, A, N)$, and similarly for the reduced bar-resolution; these maps are given by formulae

$$
\begin{aligned}
d_{0}\left(m\left[a_{1}\left|a_{2}\right| \ldots \mid a_{k}\right] n\right)= & d(m)\left[a_{1}|\ldots| a_{k}\right] n \\
& -\sum_{i=1}^{k}(-1)^{\epsilon_{i}} m\left[a_{1}\left|a_{2}\right| \ldots\left|d a_{i}\right| \ldots \mid a_{k}\right] n \\
& +(-1)^{\epsilon_{k+1}} m\left[a_{1}\left|a_{2}\right| \ldots \mid a_{k}\right] d(n), \\
d_{1}\left(m\left[a_{1}\left|a_{2}\right| \ldots \mid a_{k}\right] n\right)= & (-1)^{|m|} m a_{1}\left[a_{2}|\ldots| a_{k}\right] n \\
& +\sum_{i=2}^{k}(-1)^{\epsilon_{i}} m\left[a_{1}\left|a_{2}\right| \ldots\left|a_{i-1} a_{i}\right| \ldots \mid a_{k}\right] n \\
& -(-1)^{\epsilon_{k}} m\left[a_{1}\left|a_{2}\right| \ldots \mid a_{k-1}\right] a_{k} n,
\end{aligned}
$$

with $\epsilon_{i}=|m|+\sum_{j<i}\left|s a_{i}\right|$. An important particular case of this construction is $\bar{B}(\mathrm{k}, A$, lk), or $\bar{B}(A)$ for short, where $\mathrm{kk}$ is given the module structure from the augmentation. This is a DG coalgebra, and if $A$ is graded commutative, even a DG commutative Hopf algebra: the product in it is given by shuffles, see [16], ch. 8 for details.

Dually, for a coaugmented DG coalgebra $K=\mathbb{k} \oplus \bar{K}$, and two $K$-comodules $R$ and $L$ (right and left respectively) one defines the twosided cobar resolution $F(R, K, L)$ (resp. reduced two-sided cobar resolution $\bar{F}(R, K, L))$ as

$$
F(R, K, L)=\bigoplus_{p \geqslant 0} R \otimes\left(s^{-1} K\right)^{\otimes p} \otimes L, \quad \bar{F}(R, K, L)=\bigoplus_{p \geqslant 0} R \otimes\left(s^{-1} \bar{K}\right)^{\otimes p} \otimes L,
$$


with generic elements denoted by $r\left[k_{1}\left|k_{2}\right| \ldots \mid k_{p}\right] l$. The differential is again given by the sum $d=d_{0}+d_{1}$, where the maps

$$
d_{0}: F_{p}(R, K, L) \rightarrow F_{p}(R, K, L), \quad d_{1}: F_{p}(R, K, L) \rightarrow F_{p+1}(R, K, L),
$$

are given by the formulas

$$
\begin{aligned}
d_{0}\left(r\left[k_{1}\left|k_{2}\right| \ldots \mid k_{p}\right] l\right)= & d(r)\left[k_{1}\left|k_{2}\right| \ldots \mid k_{p}\right] l \\
& -\sum_{i=1}^{p}(-1)^{\epsilon i} r\left[k_{1}\left|k_{2}\right| \ldots\left|d k_{i}\right| \ldots \mid k_{p}\right] r \\
& +(-1)^{\epsilon_{p+1}} r\left[k_{1}\left|k_{2}\right| \ldots \mid k_{p}\right] l \\
d_{1}\left(r\left[k_{1}\left|k_{2}\right| \ldots \mid k_{p}\right] l\right)= & (-1)^{\left|r^{(0)}\right|} r^{(0)}\left[r^{(1)}\left|k_{1}\right| \ldots \mid k_{p}\right] l \\
& +\sum_{i=1}^{p}(-1)^{\epsilon_{i}+\left|k_{i}^{(1)}\right|} r\left[k_{1}|\ldots| k_{i}^{(1)}\left|k_{i}^{(2)}\right| \ldots \mid k_{p}\right] l \\
& +(-1)^{\epsilon_{p}+\left|l^{(-1)}\right|} r\left[k_{1}\left|k_{2}\right| \ldots\left|k_{p}\right| l^{(-1)}\right] l^{(0)}
\end{aligned}
$$

and similarly for the reduced case. Here $\epsilon_{i}=|r|+\sum_{j<i}\left|s^{-1} k_{j}\right|$. An important particular case of cobar resolution is $\bar{F}(K)=\bar{F}(\mathrm{k}, K$, lk), which is in a natural way a DG algebra.

\subsection{Homotopy of DG algebras and coalgebras and perturbation lemma}

If $A_{0}$ and $A_{1}$ are two DG algebras (resp. coalgebras), we say that they are homotopy equivalent, if there exists a sequence of DG algebras (resp. coalgebras) and homomorphisms

$$
A_{0} \leftarrow B_{1} \rightarrow B_{2} \leftarrow B_{3} \rightarrow \ldots \leftarrow B_{n} \rightarrow A_{1},
$$

such that all the homomorphisms in this string induce isomorphisms in homology. Such homomorphisms are called quasi-isomorphisms.

If algebras $A_{0}$ and $A_{1}$ are homotopy equivalent, their reduced bar constructions $\bar{B}\left(A_{0}\right)$ and $\bar{B}\left(A_{1}\right)$ are homotopy equivalent coalgebras. If all the intermediate elements in the string are commutative the Hopf algebra structure on $\bar{B}(A)$ is also preserved. Dually, reduced cobar-resolutions of homotopy equivalent conilpotent coalgebras are homotopy equivalent algebras.

One can replace the string above by a single map of a more general nature, the so called $A_{\infty}$ map of algebras (resp. coalgebras). One says that an $A_{\infty}$ map $\mathcal{P}$ between $A_{0}$ and $A_{1}$ is given, if there is a DG coalgebra homomorphism $\bar{B}\left(A_{0}\right) \rightarrow \bar{B}\left(A_{1}\right)$. Since $\bar{B}\left(A_{1}\right)$ is a free coalgebra, this amounts to 
a series of graded maps $\mathcal{P}(n):\left(s A_{0}\right)^{\otimes n} \rightarrow s A_{1}$, verifying certain relations, given by commutators with bar-complex differentials. For instance, first two of these relations are

$$
\begin{aligned}
d_{A_{1}}(\mathcal{P}(1)(a))= & \mathcal{P}(1)\left(d_{A_{0}} a\right), \\
\mathcal{P}(1)(a b)-\mathcal{P}(1)(a) \mathcal{P}(1)(b)= & d_{A_{1}}(\mathcal{P}(2)(a, b)) \\
& -\mathcal{P}(2)\left(d_{A_{0}} a, b\right)-(-1)^{|a|} \mathcal{P}(2)\left(a, d_{A_{0}} b\right),
\end{aligned}
$$

where we use commas instead of tensor signs; in particular, $\mathcal{P}(0)$ is a homomorphism of DG modules, which induces a homomorphism of algebras in homologies. From the point of view of the gradings in $A_{0}$ and $A_{1}$ the map $\mathcal{P}(n)$ decreases the degree by $n-1$. A particular example of $A_{\infty^{-}}$ homomorphism is given by a homomorphism of DG algebras $f$, if we put $\mathcal{P}(1)=f, \mathcal{P}(n)=0, n \geqslant 2$. The following statement can be found for example in $[22,5]$ :

Proposition 1.1. - The algebras $A_{0}$ and $A_{1}$ are homotopy equivalent, if and only if there exists an $A_{\infty}$-homomorphism $A_{0} \rightarrow A_{1}$, such that $\mathcal{P}(1)$ induces an isomorphism in homology. In particular, every such map is homotopy invertible.

If $f: A_{0} \rightarrow A_{1}$ is a quasi-isomorphism of DG algebras, $f_{\infty}^{-1}$ will denote the corresponding inverse $A_{\infty}$ map.

Many examples of $A_{\infty}$ structures are given by perturbation techniques. Let a chain map (not a homomorphism) $f^{-1}: A_{1} \rightarrow A_{0}$ and chain homotopy $H: A_{1} \rightarrow A_{1}$ be given, verifying the equations

$$
\begin{gathered}
H \circ H=0, \quad H \circ f=0, \quad f^{-1} \circ H=0, \quad f^{-1} \circ f=\operatorname{id}_{A_{0}}, \\
\quad f \circ f^{-1}-I d_{A_{1}}=d_{A_{1}, A_{1}}(H) .
\end{gathered}
$$

This is called homotopy perturbation data. Then the formulas

$$
\begin{aligned}
f_{\infty}^{-1}(n)= & f^{-1} \circ m_{A_{1}}\left(\left(H \circ m_{A_{1}}\right) \otimes \mathrm{id}_{A_{1}}+\mathrm{id}_{A_{1}} \otimes\left(H \circ m_{A_{1}}\right)\right) \ldots \\
& \ldots\left(\left(H \circ m_{A_{1}}\right) \otimes \ldots \otimes \mathrm{id}_{A_{1}}+\ldots+\mathrm{id}_{A_{1}} \otimes \ldots \otimes\left(H \circ m_{A_{1}}\right)\right) \\
& \left(H \otimes\left(f \circ f^{-1}\right) \otimes \ldots \otimes\left(f \circ f^{-1}\right)+\ldots+\mathrm{id}_{A_{1}} \otimes \ldots \otimes \mathrm{id}_{A_{1}} \otimes H\right), \\
\mathcal{H}(n)= & H \circ m_{A_{1}}\left(\left(H \circ m_{A_{1}}\right) \otimes \operatorname{id}_{A_{1}}+\mathrm{id}_{A_{1}} \otimes\left(H \circ m_{A_{1}}\right)\right) \ldots \\
& \ldots\left(\left(H \circ m_{A_{1}}\right) \otimes \ldots \otimes \mathrm{id}_{A_{1}}+\ldots+\mathrm{id}_{A_{1}} \otimes \ldots \otimes\left(H \circ m_{A_{1}}\right)\right) \\
& \left(H \otimes\left(f \circ f^{-1}\right) \otimes \ldots \otimes\left(f \circ f^{-1}\right)+\ldots+\mathrm{id}_{A_{1}} \otimes \ldots \otimes \mathrm{id}_{A_{1}} \otimes H\right),
\end{aligned}
$$

determine the homotopy inverse $A_{\infty}$ map and corresponding homotopy. 
Similarly for two coalgebras $K_{0}, K_{1}$ there exists the notion of $A_{\infty^{-}}$ homomorphisms, dual to the $A_{\infty}$-algebra homomorphism. Namely an $A_{\infty}$ coalgebra homomorphism $c \mathcal{P}: K_{0} \rightarrow K_{1}$ is a sequence of linear maps $c \mathcal{P}(n)$ : $s^{-1} K_{0} \rightarrow\left(s^{-1} K_{1}\right)^{\otimes n}$, which induce a DG algebra homomorphism of cobarresolutions $\bar{F}\left(K_{0}\right) \rightarrow \bar{F}\left(K_{1}\right)$. The maps $c \mathcal{P}(n)$ verify relations, dual to that of $A_{\infty}$ maps. There is an analogue of proposition 1.1 in this case too, which enables one invert the quasi-isomorphisms of DG coalgebras. One can also apply the perturbation lemma in this case and obtain formulae, similar to (1.2). Similar notions of homotopy equivalence can be introduced for DG modules over DG algebras and DG comodules over DG coalgebras. There are notions of $A_{\infty}$ homomorphisms of modules and comodules and analogues of the proposition 1.1.

Let $X$ be a topological space. A DG algebra $A$, homotopy equivalent to the cochain complex of $X$ will be called a model of $X$. Dually, if $G$ is a compact topological group, a DG coalgebra $K$ is called model for $G$, if it is homotopy-equivalent to the Pontrjagin coalgebra of cochains in $G$. The main example of models of both types are the de Rham complexes of smooth manifolds and the polynomial de Rham algebras of smooth algebraic groups (see section 1.5 ) respectively.

\subsection{Twisting cochains and topology of fibre bundles}

For a DG algebra $A$ and a DG complex $M$ consider an element $\phi \in \operatorname{Hom}^{1}(M, M \otimes A)$. This element determines a map

$$
d_{\phi}: M \otimes A \rightarrow M \otimes A, \quad d_{\phi}=d_{M \otimes A}+\left(\operatorname{id}_{M} \otimes m_{A}\right) \circ\left(\phi \otimes \operatorname{id}_{A}\right) ;
$$

$\phi$ is called twisting map, if $d_{\phi}^{2}=0$. Observe, that such $\phi$ induces similar differentials in tensor products $M \otimes N$ for any left DG $A$-module $N$. The tensor products, equipped with such differentials will be called twisted tensor products and denoted by $M \otimes_{\phi} N$.

If a DG module $M$ is supplemented i.e. equipped with a chain map $\epsilon: M \rightarrow \mathrm{k}$, then we associate to $\phi$ a chain map

$$
\begin{gathered}
\tilde{\phi}: M \rightarrow \bar{B}(A), \\
\tilde{\phi}(m)=\sum_{n \geqslant 0}\left(\epsilon \otimes\left(s \overline{\mathrm{id}}_{A}\right)^{\otimes n}\right) \circ\left(\phi \otimes \mathrm{id}_{A}^{\otimes n-1}\right) \circ\left(\phi \otimes \mathrm{id}_{A}^{\otimes n-2}\right) \circ \ldots \circ \phi(m) .
\end{gathered}
$$

Here $s$ denotes the natural identification of a module and its suspension. Observe that we shall either assume the finiteness conditions for the iterations of $\phi$, or pass to the completion of $\bar{B}(A)$ with respect to the tensor 
powers. Dually, for a DG coalgebra $K$ and a DG complex $L$, twisting map is an element $\psi \in \operatorname{Hom}^{1}(K \otimes L, L)$, such that the map

$$
d_{\psi}: K \otimes L \rightarrow K \otimes L, \quad d_{\psi}=d_{K \otimes L}+\left(\mathrm{id}_{K} \otimes \psi\right) \circ\left(\Delta_{K} \otimes \mathrm{id}_{L}\right)
$$

verifies the equation $d_{\psi}^{2}=0$. The same formula gives a differential on $R \otimes L$ for arbitrary right $K$-comodule $R$, which we call twisted tensor product and denote $R \otimes_{\psi} L$. If $L$ is cosupplemented (i.e. given a chain map $\eta$ : $\mathbb{k} \rightarrow L$ ), one has a chain map

$$
\tilde{\psi}: \bar{F}(K) \rightarrow L, \quad \tilde{\psi}\left(\left[k_{1}|\ldots| k_{p}\right]\right)=\psi\left(k_{1} \otimes \psi\left(k_{2} \otimes \psi\left(\ldots \psi\left(k_{p} \otimes \eta(1)\right) \ldots\right)\right)\right) .
$$

For a DG coalgebra $K$ and a DG algebra $A$ one considers the twisting maps of special kind: for $\tau \in \operatorname{Hom}^{1}(K, A)$ we put

$$
\begin{aligned}
& \phi_{\tau}: K \rightarrow K \otimes A, \quad \phi_{\tau}(k)=k^{(1)} \otimes \tau\left(k^{(2)}\right), \\
& \psi_{\tau}: K \otimes A \rightarrow A, \quad \psi_{\tau}(k \otimes a)=\tau(k) a .
\end{aligned}
$$

Then $d_{\phi_{\tau}}=d_{\psi_{\tau}}$ on $K \otimes A$ and these are twisting maps iff

$$
d_{K, A} \tau=\tau \cup \tau,
$$

where $\cup$ denotes the convolution product on $\left(\operatorname{Hom}(K, A), d_{K, A}\right)$. We shall denote the twisted differential on $K \otimes A$ by $d_{\tau}$ and the corresponding twisted tensor product by $K \otimes_{\tau} A$. The map $\tau$ is called twisting cochain, if in addition $\tau \circ \eta_{K}=0$, where $\eta_{K}$ is coaugmentation.

An important feature of such $\tau$, is that the map $\tilde{\phi_{\tau}}$ is a homomorphism of DG coalgebras, and $\tilde{\psi}_{\tau}$ is a homomorphism of DG algebras, moreover, there exist natural bijections

$$
\operatorname{DGA}(\bar{F}(K), A) \cong \operatorname{TC}(K, A) \cong \operatorname{DGC}(K, \bar{B}(A)),
$$

where TC denotes the set of twisting cochains between $K$ and $A$, DGA and DGC - the sets of homomorphisms of algebras and coalgebras respectively. For a twisting cochain $\tau$ the twisted tensor product $K \otimes_{\tau} A$ is a right DG $A$-module and left DG $K$-comodule.

Two twisting cochains $\tau, \tau^{\prime} \in \mathrm{TC}(K, A)$ are gauge-equivalent, iff there exists a graded map $c: K \rightarrow A$, invertible with respect to the convolution product and such that

$$
\tau=c^{-1} \cup \tau^{\prime} \cup c+c^{-1} \cup d_{K, A} c,
$$

$c$ is called gauge transformation. For gauge-equivalent $\tau, \tau^{\prime}, c$ the corresponding gauge transformation, one can define an isomorphism $C: K \otimes_{\tau}$ 
$A \rightarrow K \otimes_{\tau^{\prime}} A$ of DG $A$-modules and $K$-comodules, and if $\phi_{\tau}, \phi_{\tau^{\prime}}$ are the characteristic maps, then one can define chain homotopy $H_{c}$, connecting them. The opposite statement is also true in case of a connected coalgebra $K$ (i.e. $K^{0}=\mathbb{k}$ ). Thus there is an equivalence of the set of homotopy classes of $\operatorname{DGC}(K, \bar{B}(A))$ with the set of gauge-equivalence classes of the twisting cochains, refer to $[20,21]$ for details.

Let $\mathcal{P}=\{\mathcal{P}(n)\}: A_{0} \rightarrow A_{1}$ be an $A_{\infty}$ map, and $\tau \in \mathrm{TC}\left(K, A_{0}\right)$. Then if $K$ is locally nilpotent (i.e. $\Delta^{n}(k)=0$ for $n$ large enough for all $\left.k \in \bar{K}\right)$, one can define a twisting cochain $\mathcal{P}(\tau) \in \mathrm{TC}\left(K, A_{1}\right)$ by formulas

$$
\mathcal{P}(\tau)(k)=\sum_{n \geqslant 1} \mathcal{P}(n)\left(\tau\left(k^{(1)}\right) \otimes \ldots \otimes \tau\left(k^{(n)}\right)\right) .
$$

Dually, if $K_{0}, K_{1}$ are homotopy equivalent coalgebras related by $c \mathcal{P}=$ $\{c \mathcal{P}(n)\}$, one can associate to every $\tau \in \mathrm{TC}\left(K_{0}, A\right)$ a twisting cochain $c \mathcal{P}(\tau)$ :

$$
c \mathcal{P}(\tau)(k)=\sum_{n \geqslant 0} m_{n}(\underbrace{\tau \otimes \ldots \otimes \tau}_{n \text { times }}) c \mathcal{P}(n)(k)
$$

where $m_{n}$ is $n$-fold multiplication (the formula works under similar nilpotence assumptions as above). All $A_{\infty}$ homomorphisms induce maps of the twisted tensor products:

$$
1 \otimes \mathcal{P}: K \otimes_{\tau} \Omega \rightarrow K \otimes_{\mathcal{P}(\tau)} \Omega^{\prime}
$$

and similarly for $c \mathcal{P}$, the maps are given by the formulae

$$
1 \otimes \mathcal{P}(k \otimes a)=\sum_{n \geqslant 0} k^{(1)} \otimes \mathcal{P}(n)\left(\tau\left(k^{(2)}\right) \otimes \ldots \otimes \tau\left(k^{(n)}\right) \otimes a\right),
$$

The following meta-theorem (see [7, 22] for details) justifies our interest to twisting cochains

Theorem 1.2. - For every topological principal bundle $P \rightarrow X$ with structure group $G$ and all suitable models $A, K$ of $X$ and $K$, there exists a twisting cochain $\tau \in \mathrm{TC}(K, A)$ such that $K \otimes_{\tau} A$ is homotopy equivalent to $C^{*}(P)$. This twisting cochain is unique up to a gauge equivalence.

We shall not need this theorem in full generality. Below we shall give an ad hoc proof of this result in case of smooth locally trivial principal bundles over a manifold. 


\subsection{Simplicial objects, their realizations and loop spaces}

The simplicial category $\triangle$ is the category of finite nonempty linearly ordered sets and order-preserving maps; up to an isomorphism an object in $\triangle$ is equal to $\mathbf{n}=\{0 \leqslant 1 \leqslant \ldots \leqslant n\}$. All morphisms in $\triangle$ are generated by morphisms of two types:

$$
\begin{aligned}
& \partial_{i}: \mathbf{n} \rightarrow \mathbf{n}+\mathbf{1}, \partial_{i}(k)= \begin{cases}k, & k<i, \\
k+1, & k \geqslant i,\end{cases} \\
& s_{j}: \mathbf{n} \rightarrow \mathbf{n}-\mathbf{1}, s_{j}(k)=\left\{\begin{array}{ll}
k, & k \leqslant i \\
k-1, & k>i
\end{array},\right.
\end{aligned}
$$

where $i=0, \ldots, n+1, j=0, \ldots, n-1$, called faces and degeneracies respectively.

A simplicial set is a functor $X: \triangle^{o p} \rightarrow \mathcal{S}$ ets; more specifically, it is a collection $X=\left\{X_{n}\right\}_{n \geqslant 0}$ of sets and morphisms $\partial_{i}: X_{n} \rightarrow X_{n-1}, s_{j}: X_{n} \rightarrow$ $X_{n+1}, i, j=0, \ldots, n$ between them, verifying certain simplicial relations (see [17] or [22] for details). If the category $\mathcal{S}$ ets is replaced by some other category (Diff, $\mathcal{T}$ op, $\mathcal{T}_{o p^{*}}, \mathcal{M}_{\text {od }}$ etc.) one speaks about simplicial objects in the appropriate category. Morphism $F: X \rightarrow Y$ between simplicial sets is a natural transformation of the functors; it is given by a collection of maps of sets $F_{n}: X_{n} \rightarrow Y_{n}$, commuting with faces and degeneracies. Morphisms of simplicial objects in other categories are defined in the same way. The category of simplicial objects and their morphisms in $\mathcal{C}$ will be denoted $\mathscr{S C}$.

Dually, cosimplicial set, more generally, cosimplicial object in a category $\mathcal{C}$ is a covariant functor $Y: \triangle \rightarrow \mathcal{S}$ ets. It is determined by a collection of objects $Y=\left\{Y^{n}\right\}_{n \geqslant 0}$ and maps $\delta_{i}: Y^{n} \rightarrow Y^{n+1}, \sigma^{j}: Y^{n} \rightarrow Y^{n-1}, i=$ $0, \ldots, n+1, j=0, \ldots, n-1$, called cofaces and codegenracies respectively; they verify the cosimplicial relations, dual to the previous ones. Morphisms of cosimplicial objects are natural transformations of functors. Equivalently, a morphism $F: Y \rightarrow Z$ is a collection of maps $F^{n}: Y^{n} \rightarrow Z^{n}$, commuting with cofaces and codegenracies. The category of cosimplicial objects in $\mathcal{C}$ will be denoted by $c \mathscr{S C}$. A particular important example of cosimplicial space is given by $\Delta=\left\{\Delta^{n}\right\}_{n \geqslant 0}$, where $\Delta^{n}$ is the geometric $n$-simplex,

$$
\Delta^{n}=\left\{\left(t_{0}, \ldots, t_{n}\right) \in \mathbb{R}^{n} \mid t_{i} \geqslant 0, i=0, \ldots, n, \sum_{i=0}^{n} t_{i}=1\right\} .
$$

Cofaces and codegeneracies $\delta_{i}: \Delta^{n} \rightarrow \Delta^{n+1}, \sigma_{j}: \Delta^{n} \rightarrow \Delta^{n-1}$ are given by identification of the $n+1$-simplex with $i$-th face $n+1$-simplex and projection along the edge connecting the $j$-th and the $j+1$-st vertices, respectively. 
Realization of the simplicial set or simplicial space $X$ is the quotient space

$$
|X|=\coprod_{n \geqslant 0} X_{n} \times \Delta^{n} /\left\langle\left(\partial_{i} x, t\right)=\left(x, \delta_{i} t\right),\left(s_{j} x, t\right)=\left(x, \sigma_{j} t\right)\right\rangle
$$

$|X|$ is a functor from $\mathscr{S}$ Sets into $\mathcal{T}$ op. Moreover, it is an equivalence of the categories, the inverse being given by the functor of singular chains. Dually, realisation of a cosimplicial set or simplicial space $Y$ is by definition given by the following formula:

$$
|Y|=\left\{f=\left\{f_{n}\right\} \in \prod_{n \geqslant 0} \operatorname{Map}_{\mathcal{T} o p}\left(\Delta^{n}, Y^{n}\right) \mid f_{n+1}\left(\delta_{i} y\right)=\delta_{i} f_{n}(y), f_{n-1}\left(\sigma_{j} y\right)=\sigma_{j} f_{n}(y)\right\} ;
$$

this is a functor from $c \mathscr{S} \mathcal{T}$ op to $\mathcal{T}$ op. More generally, let $\Delta_{\mathcal{C}}=\left\{\Delta_{\mathcal{C}}^{n}\right\}$ be a fixed cosimplicial object in a tensor category $\mathcal{C}$. Assume that $\mathcal{C}$ has all limits and colimits. Then one can define a realization of a simplicial object $X \in \mathscr{S C}$ in a manner analogous to the realisation of simplicial sets:

$$
|X|=\coprod_{n \geqslant 0} X_{n} \times \Delta_{\mathcal{C}}^{n} / \sim
$$

where $\sim$ denotes the equivalence relation, similar to the one used before. This realisation does in general depend on the choice of the object $\Delta_{\mathcal{C}}$. See details in the books [6], [12],[17].

For a topological space $X$ with a base point $x_{0}=*$ the based loop space is the space of maps

$$
\Omega X=\Omega_{x_{0}} X=\left\{\gamma:[0 ; 1] \rightarrow X \mid \gamma(0)=\gamma(1)=x_{0}\right\}
$$

with compact-open topology; $\Omega X$ is an $H$-space with respect to the concatenation of loops. The free loop space of $X$ is the space of maps

$$
\mathcal{L} X=\left\{\gamma: S^{1} \rightarrow X\right\}
$$

The group $S^{1}$ acts on $\mathcal{L} X$ by action on arguments, and there's a Serre fibration ev $: \mathcal{L} X \rightarrow X$, given by $e v(\gamma)=\gamma(1)$ with fibre $\Omega X$. There are simplicial constructions, modeling $\Omega X$ and $\mathcal{L} X$, see [14] or [22] for references.

\subsection{Groups, connections, and characteristic classes}

Let $G \subseteq G L_{n}(\mathrm{lk})$ be a compact smooth algebraic group; $\mathfrak{g}$ its Lie algebra, $N=$ dimg. The algebra of regular functions on $G$ is the factor algebra of the 
polynomial ring over $\mathbb{k}$ of $n^{2}$ variables $u_{i j} i, j=1 \ldots n$ modulo the ideal, that determines the group; we denote it $\mathcal{A}(G)$. One should regard $u_{i j}$ as the function, which assigns to an element $g \in G$ the value of the $(i, j)^{\text {th }}$ entry of the matrix, representing it. The lk-linear homomorphisms

$$
\begin{aligned}
\Delta\left(u_{i j}\right) & =\sum_{k} u_{i k} \otimes u_{k j} \\
\epsilon\left(u_{i j}\right) & =\delta_{i j},
\end{aligned}
$$

where $\delta_{i j}$ is the Kronecker symbol, determine the bialgebra structure in $\mathcal{A}(G)$. One can introduce antipode $S$ using the Cramer's formulas for the inverse matrix. Thus we have a Hopf algebra structure on $\mathcal{A}(G)$. By PeterWeyl theorem linearly $\mathcal{A}(G)$ is equal to the direct sum

$$
\mathcal{A}(G)=\bigoplus_{\rho \in \mathcal{T}} \mathcal{A}^{\rho} .
$$

Here $\mathcal{T}$ is the set of all irreducible unitary representations $\rho: G \rightarrow U\left(n_{\rho}\right)$ of the group $G$, and $\mathcal{A}^{\rho}$ is the subspace, generated by functions $u_{i j}^{\rho}(g)=$ $\rho(g)_{i j}, i, j=1, \ldots n_{\rho}$. Observe, that the spaces $\mathcal{A}^{\rho}$ are closed under comultiplication, since

$$
\Delta\left(u_{i j}^{\rho}\right)=\sum_{k} u_{i k}^{\rho} \otimes u_{k j}^{\rho} .
$$

Polynomial de Rham algebra of $G$ is the commutative DG algebra $\Omega_{\text {poly }}(G)$, generated by $\mathcal{A}(G)$. One can extend the Hopf algebra structure to $\Omega_{\text {poly }}(G)$ by the formulas:

$$
\begin{aligned}
\Delta\left(d u_{i j}\right) & =\sum_{k}\left\{u_{i k} \otimes d u_{k j}+d u_{i k} \otimes u_{k j}\right\} \\
\epsilon\left(d u_{i j}\right) & =0,
\end{aligned}
$$

and similarly for the antipode. The differential in $\Omega_{\text {poly }}(G)$ is induced by the formula $d(f)=d f, f \in \mathcal{A}(G)$ and the Leibniz rule. Below we shall often abbreviate $\Omega_{\text {poly }}(G)$ to $\Omega_{G}$. One can show that for a compact smooth algebraic group $G$ (e.g. $G=S O(n)$ etc.) the homology of $\Omega_{\text {poly }}(G)$ is isomorphic to the singular cohomology of $G$ and this isomorphism is, in effect, isomorphism of graded Hopf algebras. The main reference for this statement is [13]. Thus $\Omega_{\text {poly }}(G)$ is a model of $G$. From this and from the classical homological algebra follows.

Proposition 1.3. - Cobar resolution of $\Omega_{\text {poly }}(G)$ is homotopy equivalent to the algebra of cochains on the classifying space $B G$. 
Let us now recall the classical Chern-Weil theory (see e.g. [18] and [23] for details). The Weil algebra of a graded Lie algebra $\mathfrak{g}$ is $W(\mathfrak{g})=$ $\widehat{S}^{*}\left(\mathfrak{g}^{*}[2]\right) \otimes \Lambda^{*}\left(\mathfrak{g}^{*}[1]\right)$, where $\widehat{S}^{*}$ and $\Lambda^{*}$ denote the completed symmetric and the exterior algebras of a vector space; we allow infinite sums of elements in the symmetric (or exterior) algebra, provided there remain only a finite sum in every finite tensor product. The elements of $\mathfrak{g}^{*}[1]$ are denoted by $a_{X^{*}}$ and the elements of $\mathfrak{g}^{*}[2]$ - by $f_{X^{*}}$, where $X^{*}$ are the corresponding elements of $\mathfrak{g}^{*}$. Let $a_{i}$ denote $a_{X_{i}^{*}}$ and $f_{i}=f_{X_{i}^{*}}$, where $X_{i}^{*}, i=1, \ldots, n$ is a basis in $\mathfrak{g}^{*}$. One puts:

$$
\begin{aligned}
\partial a_{i} & =f_{i}+\frac{1}{2} C_{j k}^{i} a_{j} a_{k}, \\
\partial f_{i} & =C_{j k}^{i} f_{j} a_{k},
\end{aligned}
$$

where $C_{i}^{j k}$ are the structure constants of the Lie algebra $\mathfrak{g}$ (i.e. for a dual base $X^{i}$ of $\left.\mathfrak{g},\left[X^{j}, X^{k}\right]=C_{i}^{j k} X_{i}\right)$. Then the algebra $W(\mathfrak{g})$ is the universal commutative differential algebra with the property: let $v: \mathfrak{g}^{*} \rightarrow \Omega^{1}$ be a linear map, $\Omega^{*}$ an arbitrary commutative DG algebra (either with finite grading, or completed in the graded sense). Then there exists a unique homomorphism of DG algebras $v^{*}: W(\mathfrak{g}) \rightarrow \Omega^{*}$, such that $v_{\left.\right|_{\mathfrak{g} *} ^{*}[1]}^{*}=v$.

The duality with $\mathfrak{g}$ and coadjoint action of $\mathfrak{g}$ on $\mathfrak{g}^{*}$ induce graded derivatives

$$
\begin{aligned}
& \theta_{X}: W(\mathfrak{g}) \rightarrow W(\mathfrak{g}), \theta_{X}\left(a_{Y^{*}}\right)=a_{a d_{X}^{*}\left(Y^{*}\right)}, \theta_{X}\left(f_{Y^{*}}\right)=f_{a d_{X}^{*}\left(Y^{*}\right)} \\
& \imath_{X}: W(\mathfrak{g}) \rightarrow W(\mathfrak{g}), \imath_{X}\left(a_{Y^{*}}\right)=Y^{*}(X), \imath_{X}\left(f_{Y}^{*}\right)=0
\end{aligned}
$$

for all $X \in \mathfrak{g}$. One checks Cartan's identities, e.g. $\left[\partial, \imath_{X}\right]=\theta_{X}$ for them. An element $\omega \in W(\mathfrak{g})$ is basic, if $\theta_{X}(\omega)=\imath_{X}(\omega)=0$ for all $X \in \mathfrak{g}$. The set of all basic elements in $W(\mathfrak{g})$ is a DG subalgebra in $W(\mathfrak{g})$. If $\mathfrak{g}$ is the Lie algebra of a compact Lie group $G$, the cohomology of the basic subalgebra $W(\mathfrak{g})^{\text {bas }}$ of its Weil algebra is isomorphic to the real cohomology of the classifying space $B G$ of $G$; the cohomology of the algebra $W(\mathfrak{g})$ is trivial.

Let $P \rightarrow X$ be a principal $G$-bundle. Unless otherwise stated, we shall assume that $G$ acts on $P$ on the left, although we shall not usually show this in formulae. Gauge bundle of $P$ is the associated group bundle $P_{A d}=$ $P \times{ }_{A d_{G}} G$, where $A d_{G}$ denotes the adjoint action of $G$ on itself. Connection $A$ on $P$ is a $\mathfrak{g}$-valued 1 -form on $P$, satisfying the covariance and normalization conditions. Let $X_{1}, \ldots, X_{N}$ be a basis of $\mathfrak{g}$, then $A=\sum_{k=1}^{N} A_{k} X_{k}, A_{k} \in$ $\Omega^{1}(P)$. For any local section $s: U \rightarrow P, U \subseteq X$, consider the inverse image $A_{U}=s^{*}(A)=\sum_{k} s^{*} A_{k} X_{k}$ of $A$. One can define connection $A$ as a collection of such locally defined $\mathfrak{g}$-valued 1-forms on $X$, also known as gauge potentials. A map $g: U \rightarrow G$ induces the gauge transformation of the 
gauge potential on $U$ :

$$
A \mapsto A^{g}=g^{-1} A g+g^{-1} d g .
$$

If $\rho$ is a representation of $G$, then we obtain a collection of local matrixvalued gauge potentials $A_{U}^{\rho}=\sum_{k} A_{k} \rho\left(h_{k}\right)$, we use the same notation for a representation of group and of the Lie algebra. In this way one defines a connection on the associated complex vector bundle $E^{\rho}=P \times_{\rho} \mathbb{C}^{n_{\rho}}$. The curvature form of the connection $A$ is

$$
F=d A-\frac{1}{2}[A, A]
$$

$F \in \Omega^{2}(P ; \mathfrak{g})$. It verifies the equivariance condition and vanishes in vertical direction. We can write $F$ as a sum

$$
F=\sum_{i} F^{i} \otimes X_{i}, \quad F^{i}=d A^{i}-\sum_{i<j} C_{j k}^{i} A^{j} \wedge A^{k},
$$

where $C_{j k}^{i}$ are the structure constants of the Lie algebra $\mathfrak{g} ; F$ verifies the Bianchi identity

$$
d F=[F, A]
$$

and locally $F$ is a collection of $\mathfrak{g}$-valued 2-forms on trivializing cover $U$; $g: U \rightarrow G$ induces the transformation

$$
F \mapsto F^{g}=g^{-1} F g .
$$

The linear map $A$, given by the connection

$$
X^{*} \mapsto X^{*}(A) \in \Omega^{1}(P),
$$

by the universal property of $W(\mathfrak{g})$ gives a DG homomorphism (Chern-Weil homomorphism) $c w: W(\mathfrak{g}) \rightarrow \Omega^{*}(P)$, such that $c w\left(W(\mathfrak{g})^{\text {bas }}\right) \subseteq \Omega^{*}(P)^{\text {bas }}=$ $\Omega^{*}(X)$. This map is called the Chern-Weil map, its image in cohomology of the base is the algebra of characteristic classes of $P$.

\section{Twisting cochains and characteristic classes}

In this section we give few constructions of the twisting cochains, associated with an arbitrary principal bundle $P$ over a smooth closed manifold $X$. Our major examples of twisting cochains will take values in the Čech-de Rham complex of the base. We shall also show the relation of our constructions and the Chern classes given by the Chern-Weil homomorphism, associated with a connection on the principal bundle. This chapter is closely related to the paper [23], where we give few details, omitted here and also discuss the relation of our construction and some previously known results. After this we describe a simple way to "globalize" our constructions so as to obtain the twisting cochains with values in de Rham algebra or some other commutative DG model of the base. 


\section{G. Sharygin}

\subsection{Twisting cochain and Čech cohomology}

Let $P \stackrel{G}{\rightarrow} X$ be a principal $G$-bundle with smooth algebraic structure group $G$ and compact $X$. We are going to construct the twisting cochain, associated to this bundle, determined on the Hopf algebra of the polynomial functions on $G$ (see section 1.5). This cochain will take values in the algebra of Cech cochains on $X$ with values in the sheaf of de Rham differential forms on $X$. Our ideas are based on the work of Shih, [24].

Let us begin with the description of the Čech complex. Let $\mathcal{U}=\left\{U_{\alpha}\right\}_{\alpha \in A}$ be a trivializing cover of $X$, where $A$ is a finite linearly ordered set. Then $P_{\mid U_{\alpha}}$ is trivial for all $\alpha$. We fix the isomorphisms of principal bundles $\varphi_{\alpha}$ : $\pi^{-1}\left(U_{\alpha}\right)=P_{\mid U_{\alpha}} \cong G \times U_{\alpha}$. Then the cocycle $\left\{g_{\alpha \beta}\right\}_{\alpha \preceq \beta}$, that determines $P$, can be identified with the maps $\varphi_{\alpha}^{-1} \circ \varphi_{\beta}$ (here we identify the isomorphism $\varphi_{\alpha}$ and its restriction to $U_{\alpha \beta}=U_{\alpha} \cap U_{\beta}$.)

Consider the open cover of $P$ by cylindrical sets $\mathcal{V}=\left\{V_{\alpha}=\pi^{-1}\left(U_{\alpha}\right)\right\}$. Then the complex of Cech cochains on $P$, associated with $\mathcal{V}$ is:

$$
\begin{gathered}
\check{C}^{n}\left(\mathcal{V}, \Omega_{D R}(V)\right)=\left\{h_{\alpha_{0}, \ldots, \alpha_{p}} \in \Omega_{D R}^{q}\left(V_{\alpha_{0}, \ldots, \alpha_{p}}\right) \mid \alpha_{0} \prec \ldots \prec \alpha_{p},\right. \\
\left.\alpha_{i} \in A, i=0, \ldots, p, p+q=n\right\}
\end{gathered}
$$

where $V_{\alpha_{0}, \ldots, \alpha_{p}}=V_{\alpha_{0}} \cap \ldots \bigcap V_{\alpha_{p}}$. The differential on $\check{C}^{n}\left(\mathcal{V}, \Omega_{D R}(V)\right)$ is given by the sum of de Rham differentials on $V_{\alpha}$ and the Cech differential

$$
\delta(\{h\})_{\mid V_{\alpha_{0}, \ldots, \alpha_{p+1}}}=\sum_{i=0}^{p+1}(-1)^{i} h_{\alpha_{0}, \ldots, \widehat{\alpha_{i}}, \ldots, \alpha_{p+1}} .
$$

It is easy to show, that the cohomology of $\check{C}^{n}\left(\mathcal{V}, \Omega_{D R}(V)\right)$ is isomorphic to the de Rham cohomology of $P$. One can use induction on the number of elements in $\mathcal{V}$ and apply Mayer-Vietoris exact sequence. There is an algebra structure on $\check{C}^{n}\left(\mathcal{V}, \Omega_{D R}(V)\right)$ (see the formula (2.4) below) and the isomorphism of cohomology, commutes with the multiplications. The details can be found in the papers [23], [10], book [3] and section 2.4 below.

Isomorphisms $\varphi_{\alpha}$ allow one identify $\Omega_{D R}^{*}\left(V_{\alpha}\right)$ with tensor products $\Omega_{D R}^{*}(G) \otimes \Omega_{D R}^{*}\left(U_{\alpha}\right)$. We restrict the isomorphisms $\varphi_{\alpha_{0}}$ to $\Omega_{D R}^{*}\left(V_{\alpha_{0}, \ldots, \alpha_{p}}\right)$; recall, that the set $A$ is ordered and every time we consider an intersection $U_{\alpha_{0}} \cap \ldots \cap U_{\alpha_{k}}$ we suppose $\alpha_{0} \prec \ldots \prec \alpha_{p}$. Combining these isomorphisms we obtain an isomorphism of graded spaces

$$
\nabla: \check{C}^{n}\left(\mathcal{V}, \Omega_{D R}(V)\right) \cong \Omega_{D R}^{*}(G) \otimes \check{C}^{n}\left(\mathcal{U}, \Omega_{D R}(U)\right) .
$$

$\nabla$ is neither an isomorphism of algebras, nor does it commute with differentials, so one can consider two differentials on $\Omega_{D R}^{*}(G) \otimes \check{C}^{n}\left(\mathcal{U}, \Omega_{D R}(U)\right)$ : 
one induced from $\check{C}^{n}\left(\mathcal{V}, \Omega_{D R}(V)\right)$ with the help of $\nabla$, and the other one is the tensor product of differentials. Denote the former one by $d_{P}$ and the latter by $d$. Then $d=1 \otimes d_{U}+1 \otimes \delta^{\prime}+d_{G} \otimes 1$, where $d_{U}, d_{G}$ are the de Rham differentials on $U \subseteq X$ and $G$ respectively, and $\delta^{\prime}$ is the Čech differential on $\check{C}^{n}\left(\mathcal{U}, \Omega_{D R}(U)\right)$.

Our purpose is to define a twisting cochain $\phi$ on $\Omega_{\text {poly }}(G)$ with values in $\check{C}^{n}\left(\mathcal{U}, \Omega_{D R}(U)\right)$ such that $d_{P}=d_{\phi}$ on $\Omega_{G} \otimes \check{C}^{n}\left(\mathcal{U}, \Omega_{D R}(U)\right)$ (this complex is equivalent to $\Omega_{D R}^{*}(G) \otimes \check{C}^{n}\left(\mathcal{U}, \Omega_{D R}(U)\right)$ because of our assumptions on $G)$. To this end consider the following map (Ch. 2, §1, [24]):

$\Omega_{G} \stackrel{e}{\longrightarrow} \Omega_{G} \otimes \check{C}^{n}\left(\mathcal{U}, \Omega_{D R}(U)\right) \stackrel{d_{p}-d}{\longrightarrow} \Omega_{G} \otimes \check{C}^{n}\left(\mathcal{U}, \Omega_{D R}(U)\right) \stackrel{\epsilon \otimes 1}{\longrightarrow} \check{C}^{n}\left(\mathcal{U}, \Omega_{D R}(U)\right)$.

Here $e(\omega)=\omega \otimes 1$ and $\epsilon: \Omega_{G} \rightarrow \mathbb{C}$ is the counit in bialgebra $\Omega_{G}$. We shall denote the map (2.1) by $\phi_{P}$.

Proposition 2.1. - The map $\phi_{P}$ is a twsting cochain, i.e. the equation (1.3) holds and $\phi_{P}(1)=0$.

Proof. - Let us first of all find an explicit formula for $\phi_{P}$. Since the coproduct on $\Omega_{G}$ is induced from that on $\Omega_{G}^{0}=\mathcal{A}(G)$, and the isomorphisms induced by $\varphi_{\alpha}$ (as well as the maps $e$ and $\epsilon \otimes 1$ ) intertwine the de Rham differentials, it is enough to do it, and to check the equation (1.3) only for functions on $G$.

We recall that $1 \in \check{C}^{0}\left(\mathcal{U}, \Omega_{D R}(U)\right)$ is given by the degree 0 Cech cochain that is equal to the constant function $1_{\alpha} \equiv 1$ on every open subset $U_{\alpha} \in \mathcal{U}$. So for arbitrary $f \in \mathcal{A}(G)$ we have

$$
d(e(f))=d\left(f \otimes\left\{1_{\alpha}\right\}\right)=d_{G} f \otimes\left\{1_{\alpha}\right\},
$$

since $d_{U}\left(\left\{1_{\alpha}\right\}\right)=\delta^{\prime}\left(\left\{1_{\alpha}\right\}\right)=0$. On the other hand,

$$
d_{P}(e(f))=d_{P}\left(f \otimes\left\{1_{\alpha}\right\}\right)=\left(\left\{\varphi_{\alpha}^{*}\right\}\right)^{-1}\left(d_{V}+\delta\right)\left(\left\{\varphi_{\alpha}^{*}\left(f \otimes 1_{\alpha}\right)\right\}\right)
$$

But since $d_{V} \varphi_{\alpha}^{*}=\varphi_{\alpha}^{*}\left(d_{G} \otimes 1+1 \otimes d_{U}\right)$, we conclude, that

$$
\begin{aligned}
d_{P}(e(f))-d(e(f)) & =\left(\left\{\varphi_{\alpha}^{*}\right\}\right)^{-1} \delta\left(\left\{\varphi_{\alpha}^{*}\left(f \otimes 1_{\alpha}\right)\right\}\right) \\
& =\left\{\left(\varphi_{\alpha}^{*}\right)^{-1}\left(\varphi_{\alpha}^{*}\left(f \otimes 1_{\alpha}\right)-\varphi_{\beta}^{*}\left(f \otimes 1_{\beta}\right)\right)\right\}_{\alpha \prec \beta} \\
& =\left\{f \otimes 1_{\alpha}-\left(\varphi_{\beta} \varphi_{\alpha}^{-1}\right)^{*}\left(f \otimes 1_{\beta}\right)\right\}_{\alpha \prec \beta} .
\end{aligned}
$$

Now recall, that the homeomorphism $\varphi_{\beta} \varphi_{\alpha}^{-1}: G \times U_{\alpha \beta} \rightarrow G \times U_{\alpha \beta}$ is given by $(g, x) \mapsto\left(g g_{\alpha \beta}(x), x\right)$ and $\epsilon(f)=f(e)$, where $e$ is the unit in $G$. So we conclude that

$$
\phi_{P}(f)=\left\{f(e) 1_{\alpha \beta}-f \circ g_{\alpha \beta}\right\}_{\alpha \prec \beta} .
$$


Now it is evident that $\phi_{P}(1)=0$. In case when $f$ is replaced with a differential form $\omega$ of degree greater than 0

$$
\phi_{P}(\omega)=\left\{1_{\alpha \beta} 1^{*} \omega-g_{\alpha \beta}^{*} \omega\right\}_{\alpha \prec \beta}=-\left\{g_{\alpha \beta}^{*} \omega\right\}_{\alpha \prec \beta} .
$$

Now we are able to check the equation (1.3). Recall that the comultiplication in $\Omega_{G}$ is induced from the product of matrices, so that the following relation holds

$$
\sum \omega^{(1)}(g) \omega^{(2)}(h)=\omega(g h), \omega \in \Omega_{G}, g, h \in G .
$$

Also recall, that the multiplication in $\check{C}\left(\mathcal{U}, \Omega_{D R}(U)\right)$ is given by the formula $\left(h^{\prime} \cup h^{\prime \prime}\right)_{\alpha_{0}, \ldots, \alpha_{p+q}}=(-1)^{p\left|h^{\prime \prime}\right|_{2}}\left(h_{\alpha_{0}, \ldots, \alpha_{p}}^{\prime}\right)_{\mid U_{\alpha_{0}, \ldots, \alpha_{p+q}}} \cdot\left(h_{\alpha_{p}, \ldots, \alpha_{p+q}}^{\prime \prime}\right)_{\mid U_{\alpha_{0}, \ldots, \alpha_{p}+q}}$,

where $\left|h^{\prime \prime}\right|_{2}$ is the second (de Rham) degree of $h^{\prime \prime}$. Let us denote the differential in $\check{C}\left(\mathcal{U}, \Omega_{D R}(U)\right)$ by $d_{B}=d_{U}+\delta^{\prime}$. We compute

$$
\begin{aligned}
d_{B} \phi_{P}(f)-\phi_{P}\left(d_{G} f\right) & =\left(d_{U}+\delta^{\prime}\right) \phi_{P}(f)-\phi_{P}\left(d_{G} f\right) \\
& =\delta^{\prime}\left\{f(e) 1_{\alpha \beta}-f \circ g_{\alpha \beta}\right\}_{\alpha \prec \beta} .
\end{aligned}
$$

Here we used the equation $d_{U} f\left(g_{\alpha \beta}\right)=g_{\alpha \beta}^{*} d_{G} f$. Further,

$$
\begin{aligned}
& \delta^{\prime}\left\{f(e) 1_{\alpha \beta}-f \circ g_{\alpha \beta}\right\}_{\alpha \prec \beta}=\left\{f(e) 1_{\alpha \beta \gamma}-\left(f \circ g_{\beta \gamma}\right)_{\mid U_{\alpha \beta \gamma}}\right\}_{\alpha \prec \beta \prec \gamma} \\
& -\left\{f(e) 1_{\alpha \beta \gamma}-\left(f \circ g_{\alpha \gamma}\right)_{\mid U_{\alpha \beta \gamma}}\right\}_{\alpha \prec \beta \prec \gamma}+\left\{f(e) 1_{\alpha \beta \gamma}-\left(f \circ g_{\alpha \beta}\right)_{\mid U_{\alpha \beta \gamma}}\right\}_{\alpha \prec \beta \prec \gamma} \\
& \quad=\left\{f(e) 1_{\alpha \beta \gamma}-\left(f \circ g_{\alpha \beta}\right)_{\mid U_{\alpha \beta \gamma}}+\left(f \circ g_{\alpha \gamma}\right)_{\mid U_{\alpha \beta \gamma}}-\left(f \circ g_{\beta \gamma}\right)_{\mid U_{\alpha \beta \gamma}}\right\}_{\alpha \prec \beta \prec \gamma}
\end{aligned}
$$

Next, we compute $\phi_{P} \cup \phi_{P}(f)$ :

$$
\begin{aligned}
& \phi_{P} \cup \phi_{P}(f)=\left\{f^{(1)}(e) 1_{\alpha \beta}-f^{(1)} \circ g_{\alpha \beta}\right\}_{\alpha \prec \beta} \cup\left\{f^{(2)}(e) 1_{\alpha \beta}-f^{(2)} \circ g_{\alpha \beta}\right\}_{\alpha \prec \beta} \\
& =\left\{f(e) 1_{\alpha \beta \gamma}-\left(f \circ g_{\alpha \beta}\right)_{\mid U_{\alpha \beta \gamma}}-\left(f \circ g_{\beta \gamma}\right)_{\mid U_{\alpha \beta \gamma}}+\left(f \circ g_{\alpha \gamma}\right)_{\mid U_{\alpha \beta \gamma}}\right\}_{\alpha \prec \beta \prec \gamma} .
\end{aligned}
$$

Here we have used the relations $(2.3),(2.4)$ and the cocycle relation $g_{\alpha \beta} g_{\beta \gamma}=$ $g_{\alpha \gamma}$. Now it is evident that (1.3) holds for $\phi_{P}$.

The next theorem is the main result of this paragraph. It is similar to that of the Theorem 2, of Ch. 2 [24].

THEOREM 2.2. - On the tensor product $\Omega_{G} \otimes \check{C}^{*}\left(\mathcal{U}, \Omega_{D R}(U)\right)$ one has

$$
d_{P}=d_{\phi_{P}}=d+\phi_{P} \cap 1
$$


Proof. - The simplest way to demonstrate this is by a direct inspection of formulas:

$\left(d+\phi_{P} \cap 1\right)\left(\omega \otimes\left\{h_{\alpha_{1} \ldots \alpha_{n}}\right\}\right)=d_{G} \omega \otimes\left\{h_{\alpha_{1} \ldots \alpha_{n}}\right\}+(-1)^{|\omega|} \omega \otimes\left\{d_{U} h_{\alpha_{1} \ldots \alpha_{n}}\right\}$

$+(-1)^{|\omega|} \omega \otimes \delta_{U}\left\{h_{\alpha_{1} \ldots \alpha_{n}}\right\}-(-1)^{|\omega|} \omega^{(1)} \otimes\left\{\omega^{(2)}(e) 1_{\alpha \beta}-g_{\alpha \beta}^{*} \omega^{(2)}\right\} \cup\left\{h_{\alpha_{1} \ldots \alpha_{n}}\right\}$

Further, $\delta^{\prime}\left\{h_{\alpha_{1} \ldots \alpha_{n}}\right\}=\left\{1_{\alpha \beta}\right\} \cup\left\{h_{\alpha_{1} \ldots \alpha_{n}}\right\}$ and $\omega^{(1)} \cdot \omega^{(2)}(e)=\omega$, while $\omega^{(1)} \cdot g_{\alpha \beta}^{*} \omega^{(2)}=\left(\varphi_{\beta} \varphi_{\alpha}^{-1}\right)^{*} \omega$. Thus the expression on the right side of (2.8) is equal to

$d_{G} \omega \otimes\left\{h_{\alpha_{1} \ldots \alpha_{n}}\right\}+(-1)^{|\omega|} \omega \otimes\left\{d_{U} h_{\alpha_{1} \ldots \alpha_{n}}\right\}+(-1)^{|\omega|}\left(\varphi_{\beta} \varphi_{\alpha}^{-1}\right)^{*} \omega \otimes\left\{h_{\alpha_{1} \ldots \alpha_{n}}\right\}$

$=d_{P}$.

Remark 2.3. - It is not difficult to show that if $g_{\alpha \beta}$ and $g_{\alpha \beta}^{\prime}$ are two cohomologous noncommutative 1-cocycles with values in $G$, then the twisting cochains, determined by them are gauge-equivalent. Recall that cocycles $g_{\alpha \beta}$ and $g_{\alpha \beta}^{\prime}$ are cohomologous, iff there exist maps $h_{\alpha}: U_{\alpha} \rightarrow G$ such that $\left(h_{\alpha}\right)_{U_{\alpha \beta}} g_{\alpha \beta}=g_{\alpha \beta}^{\prime}\left(h_{\beta}\right)_{\left.\right|_{\alpha \beta}}$. Then the following formula defines a gauge transformation $c_{h}$ connecting the corresponding twisting cochains: $\Omega_{G} \ni k \stackrel{c_{h}}{\mapsto}\left\{h_{\alpha}^{*}(k)\right\}$.

\subsection{Twisting cochains and Chern-Weil classes}

We are going to discuss the relation of the twisting cochain $\phi_{P}$ and the Chern-Weil construction of characteristic classes. A more detailed treatment of this subject can be found in [23], where we also compare the construction, presented here, with previously-known ones (that of Bott and Dupont, see $[3,10]$.

Consider a twisting cochain $\phi$ on a coaugmented DG coalgebra $K$ with values in a DG algebra $A$. Recall that coaugmentation is a homomorphism $\eta: \mathbb{k} \rightarrow K$, where $\mathbb{k}$ is the trivial 1-dimensional differential coalgebra, generated by a group-like element 1 vanishing under differential. We shall identify $1 \in \mathbb{k}$ and its image $\eta(1) \in K$. Thus coaugmenting is equivalent to choosing a closed group-like element $1 \in K$, twisting cochain $\phi$ should send 1 to 0 . One calls $\omega \in K$ primitive, if $\Delta(\omega)=\omega \otimes 1+1 \otimes \omega$, where $\Delta$ is the coproduct in $K$. Let $\operatorname{Pr}^{*}(K)$ denote the space of primitive elements in $K$, $\operatorname{Pr}^{*}(K)$ is a subcomplex in $K$. From (1.3) it follows that $\phi$ defines a degree 1 chain map $\phi: \operatorname{Pr}^{*}(K) \rightarrow A^{*+1}$ and the maps from $\operatorname{Pr}^{*} K$ to $A$, induced by $\phi$ and its gauge-equivalent $\phi^{\prime}$ are homotopic. More generally, the twisting cochain defines a homomorphism of differential graded algebras $\tilde{\phi}: \bar{F}(K) \rightarrow$ 
$A$ and the chain homotopy class of this map does not change with the action of gauge transformations (see section 1.3). The map $\phi: \operatorname{Pr}^{*}(K) \rightarrow A^{*+1}$ we consider, is equal to the composition of $\tilde{\phi}$ with the inclusion of $\operatorname{Pr}^{*}(K)$ to $\bar{F}(K)$ (which commutes with differential). From section 1.3 (see also [7]) it follows that the map $\tilde{\phi}$ is a cohomological counterpart of the classifying map $f: X \rightarrow B G$ of the bundle $P$. Characteristic classes of $P$ are given by the pullbacks $f^{*}(c)$, where $c$ is a class in $H^{*}(B G)$. Thus the problem of finding the characteristic class, associated by $f^{*}$ with $c \in H^{*}(B G)$ is reduced to finding a closed cocycle in $F(K)$ which corresponding to $c$ under the isomorphism of cohomology.

Let us give an example of such formula. Let $K=\Omega_{G}, \omega=g^{-1} d g$ be the left-invariant $\mathfrak{g}$-valued Maurer-Cartan form on $G$, and $\tilde{\omega}=(d g) g^{-1}$ - its right invariant counterpart. Here $g$ is the generic element of $G$. We regard $g$ as a matrix-valued function on $G$, associating to $x \in G$ its matrix form with respect to a fixed basis. Identify $g$ with the element $\left(u_{i j}\right)$ of $\operatorname{Mat}_{n}(\mathcal{A}(G))$, then $\omega$ and $\tilde{\omega}$ are matrix-valued differential 1-forms. Consider the form $\omega_{3}=\operatorname{Tr}(\omega \wedge \omega \wedge \omega)=\omega_{i}^{k} \wedge \omega_{k}^{l} \wedge \omega_{l}^{i}$ (the summation is over the repeating indices). This element is closed, but not primitive. It is easy to check this with the help of the following formulas (the first one is the Maurer-Cartan equation, and the second follows directly from the definition of coproduct)

$$
d \omega_{i}^{j}=-\omega_{i}^{k} \wedge \omega_{k}^{j}
$$

and

$$
\Delta\left(\omega_{i}^{j}\right)=\omega_{k}^{l} \otimes g_{i}^{k} g_{l}^{j}+1 \otimes \omega_{i}^{j} .
$$

However, although not primitive, $\omega_{3}$ is biinvariant with respect to the action of $G$. As one knows (see e.g. [18]) such differential forms generate the cohomology of $G$. One can show that the corresponding element in cohomology is primitive and hence it corresponds to a class in the cohomology of $B G$. However, $\omega_{3}$ doesn't give a cocycle in $\bar{F}(K)$, if we map $\Omega_{G}=K$ into $\bar{F}(K)$ as the space of 1 -tensors: one should modify $\omega_{3}$ inside $\bar{F}(K)$ by a correction term, with tensor degree 2 so that the result be closed. Take $\hat{\omega}_{3} \in \bar{F}(K)$ equal to $\omega_{3}-3 \operatorname{Tr}(\omega \otimes \tilde{\omega})$, where

$$
\operatorname{Tr}(\omega \otimes \tilde{\omega})=\omega_{i}^{k} \otimes \tilde{\omega}_{k}^{i},
$$

the summation is over the repeating indices. Formulae (2.9) and (2.10) (and similar formulae for $\tilde{\omega})$ are used to check that $\hat{\omega}_{3}$ is a cocycle in $\bar{F}(K)$. If we apply the map $\tilde{\phi}$ (where $\phi=\phi_{P}$ is the twisting cochain from the previous paragraph) to this element, we obtain the Čech form

$$
\left\{\operatorname{Tr}\left(g_{\alpha \beta}^{-1} d g_{\alpha \beta} \wedge g_{\alpha \beta}^{-1} d g_{\alpha \beta} \wedge g_{\alpha \beta}^{-1} d g_{\alpha \beta}\right)\right\}_{\alpha \prec \beta}-3\left\{\operatorname{Tr}\left(g_{\alpha \beta}^{-1} d g_{\alpha \beta} \wedge d g_{\beta \gamma} g_{\beta \gamma}^{-1}\right)\right\}_{\alpha \prec \beta \prec \gamma} .
$$


This cochain is closed, which can be shown by a direct calculation, which we omit. Let us denote this cocycle by $\tilde{c}_{2}$. There exist many ways to show that this element corresponds to a characteristic class of $P$. We shall just specify a Čech cochain $a=a\left(g_{\alpha \beta}, A, F\right)$, where $A, F$ are a connection in the principal bundle $P$ and its curvature, such that

$$
(d+\delta)(a)=3\{\operatorname{Tr}(F \wedge F)\}_{\alpha}-\tilde{c}_{2} .
$$

We put

$$
a\left(g_{\alpha \beta}, A, F\right)=\left\{\operatorname{Tr}\left(3 F_{\alpha} \wedge A_{\alpha}-A_{\alpha}^{\wedge 3}\right)\right\}_{\alpha}-\left\{A_{\alpha} \wedge d g_{\alpha \beta} g_{\alpha \beta}^{-1}\right\}_{\alpha \prec \beta},
$$

where $A_{\alpha}$ and $F_{\alpha}$ are the local gauge potential and curvature respectively (i.e. gauge potential in the open set $\left.U_{\alpha}\right)$. Use the Bianchi identities ((1.12) and (1.13)) and the gauge transformation formulas ((1.11) and (1.14)) to obtain (2.12) by a direct calculation.

It is possible to show that there always exists a cochain, relating the forms associated with the twisted cochain $\phi_{P}$, and the usual Chern-Weil forms. To this end consider the Weil algebra $W(\mathfrak{g})$ of $\mathfrak{g}$ (section 1.5); $\mathfrak{g}$ acts on $W(\mathfrak{g})$ by the derivations $\theta_{X}$. Let us integrate this action to the action of the group $G$. We assumed that $G$ was an algebraic group, thus the action of $G$ on $\mathfrak{g}^{*}$ is algebraic in the following sense: there is a homomorphism of algebras $\Delta_{W}: \mathfrak{g}^{*} \rightarrow \mathcal{A}(G) \otimes \mathfrak{g}^{*}$, such that

$$
g\left(X^{*}\right)=\Delta_{W}\left(X^{*}\right)(g) .
$$

Here we regard $\Delta_{W}\left(X^{*}\right)$ as a $\mathfrak{g}^{*}$-valued function on $G$. We extend $\Delta_{W}$ to a map $\tilde{\Delta}_{W}: \mathfrak{g}^{*} \rightarrow \mathcal{A}(G) \otimes \mathfrak{g}^{*} \oplus \Omega_{G}^{1} \otimes 1 \subset\left(\Omega_{G} \otimes W(\mathfrak{g})\right)^{1}$ by the formula

$$
\tilde{\Delta}_{W}\left(X^{*}\right)=\Delta_{W}\left(X^{*}\right)+\omega_{X^{*}} \otimes 1,
$$

where $\omega_{X^{*}}$ is the unique right-invariant differential 1-form on $G$, which coincides with $X^{*}$ in the unit of $G$. From the universality of $W(\mathfrak{g})$, it follows that there exists a unique extension $\tilde{\Delta}_{W}^{*}: W(\mathfrak{g}) \rightarrow \Omega_{D R}^{*}(G) \otimes W(\mathfrak{g})$ of $\tilde{\Delta}_{W}$ to a homomorphism of differential algebras, then $\omega \in W(\mathfrak{g})$ is basic iff $\tilde{\Delta}_{W}^{*}(\omega)=1 \otimes \omega$. Thus $W(\mathfrak{g})$ is a (left) $\Omega_{\text {poly }}^{*}(G)$ differential comodule. We can form the reduced cobar resolution of $W(\mathfrak{g})$ as $\Omega_{G}$-comodule,

$$
F(W)=\bar{F}\left(W(\mathfrak{g}), \Omega_{G}, \mathrm{k}\right)=\bigoplus_{n \geqslant 0} \bar{\Omega}_{G}^{\otimes n} \otimes W(\mathfrak{g}) .
$$

Both $\bar{F}\left(\Omega_{G}\right)$ and $W(\mathfrak{g})^{\text {bas }}$ can be embedded into $F(W)$ as subcomplexes: in the former case we use the map

$$
\begin{gathered}
\left.i_{1}:\left[a_{1} \mid \ldots\right] a_{n}\right] \mapsto 1\left[a_{1}|\ldots| a_{n}\right], \\
-317-
\end{gathered}
$$


and in the latter case the map

$$
i_{2}: \omega \mapsto \omega[] .
$$

The following proposition is an important technical step to the proof of the claim above:

Proposition 2.4. - The cohomology of $F(W)$ is isomorphic (as vector space) to the real-valued cohomology of BG. Moreover, the inclusions (2.15) and (2.16) give isomorphisms on cohomologies.

Proof. - This statement seems to be generally known so we give only a sketch here, see [23] for further details. First we consider the following filtration of $F(W)$ :

$$
F_{p}^{\prime}(F(W))=\left\{\left[x_{1}|\ldots| x_{n}\right] \omega \in F(W)\left|x_{i} \in \bar{\Omega}_{G}, \omega \in W(\mathfrak{g}),\right| \omega \mid \leqslant p\right\}
$$

for $p \geqslant 0$, i.e. this is just the filtration, associated to the degrees of $W(\mathfrak{g})$. The associated spectral sequence can be easily computed: $E_{0}^{\prime}=g r F_{p}^{\prime}(F(W))=$ $T\left(s^{-1} \bar{\Omega}_{G}\right) \otimes W(\mathfrak{g})$ as linear space (here $T(V)$ is the tensor algebra of a vector space $V$ ) and the differential is equal to $1 \otimes \partial$. But $W(\mathfrak{g})$ is acyclic in degrees greater than 0 , thus ${E^{\prime}}_{1}^{p 0}=T\left(\bar{\Omega}_{G}\right)^{p}, E_{1}^{p q}=0, q \geqslant 1$ and the differential in $T\left(\bar{\Omega}_{G}\right)$ is equal to the usual differential in the cobar construction, where we identify $T\left(\bar{\Omega}_{G}\right)$ with $\bar{F}\left(\Omega_{G}\right)$ in an obvious way. Hence, the spectral sequence collapses at $E_{2}$-term, and its cohomology is equal to the cohomology of $\bar{F}\left(\Omega_{G}\right)$, moreover the inclusion (2.15) establishes an isomorphism in cohomology.

Now consider another filtration $F^{\prime \prime}$ on $F(W)$, defined as follows

$$
F_{p}^{\prime \prime}(F(W))=\left\{\left[x_{1}|\ldots| x_{n}\right] \omega \in F(W) \mid n \leqslant p\right\} .
$$

The associated grading is by the number of tensors in the tensor product and hence the $E_{1}$-term of the associated spectral sequence (which is equal to the cohomology of $F(W)$ with respect to the second part of its differential) can be identified with the cohomology of $W(\mathfrak{g})$ as the (left) $\Omega_{G}$-comodule, non-differential.

In order to calculate this cohomology, consider the exponential map $\exp : \mathfrak{g} \rightarrow G$. As it is well-known, if we identify $\mathfrak{g}$ with the space of leftinvariant vector fields on $G$, this map will intertwine the (right) action of $G$ on itself by translations and its adjoint action on $\mathfrak{g}$. Then the inverse image of exp determines a homomorphism from $\mathcal{A}(G)$ (the algebra of polynomial or power series functions on $G$ ) to the algebra of formal power series functions 
on $\mathfrak{g}$, compatible with the action of $G$. But this latter algebra is naturally isomorphic to $\widehat{S}^{*}\left(\mathfrak{g}^{*}\right)$. So, ignoring the grading, we obtain a homomorphism $\mathcal{A}(G) \rightarrow \widehat{S}^{*}\left(\mathfrak{g}^{*}[2]\right)$, compatible with the coaction of $\mathcal{A}(G)$ and hence, as it is easy to check, with coaction of $\Omega_{G}$. If we combine this map with the evident identification of the differential forms on $G$, regarded as a $G$-module, with $\mathcal{A}(G) \otimes \Lambda\left[\omega_{X_{1}^{*}}, \ldots, \omega_{X_{n}^{*}}\right] \cong \mathcal{A}(G) \otimes \Lambda^{*}\left(\mathfrak{g}^{*}[1]\right)$ which is due to the fact that every group is parallelisable, (see also the general theory of differential calculi on Hopf algebras, [26]) we obtain a homomorphism $\Omega_{D R}(G) \rightarrow W(\mathfrak{g})$, compatible with coaction of $\Omega_{G}$ on both sides. Hence, $W(\mathfrak{g})$ turns into a Hopf-module over the Hopf algebra $\Omega_{G}$ (see [25] for the definition and properties of Hopf-modules.)

It follows from a well-known property of Hopf-modules, that $W(\mathfrak{g}) \cong$

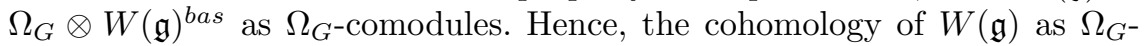
comodule is equal to $W(\mathfrak{g})^{\text {bas }}$ in tensor degree 0 and 0 otherwise, recall that here we neglect the grading in $W(\mathfrak{g})$. So $E_{1}^{\prime \prime}{ }_{1}^{p 0}=\left(W(\mathfrak{g})^{\text {bas }}\right)^{p},{E^{\prime \prime}}_{1}^{p q}=0, q \geqslant$ 1 . Hence this spectral sequence also collapses at $E_{2}$-term and converges to the cohomology of $W(\mathfrak{g})^{\text {bas }}$. Moreover, the inclusion of (2.16) establishes the isomorphism in cohomology.

Now we can define a map $c_{\phi}: F(W) \rightarrow \check{C}\left(\mathcal{U}, \Omega_{D R}(U)\right)$, combining the Chern-Weil map on $W(\mathfrak{g})$ and the map, determined by the twisting cochain on the cobar resolution. Namely, put

$$
c_{\phi}\left(\left[x_{1}|\ldots| x_{n}\right] \omega\right)=\phi_{P}\left(x_{1}\right) \cup \ldots \cup \phi_{P}\left(x_{n}\right) \cup\left\{c w_{\alpha}(\omega)\right\}_{\alpha},
$$

where $c w_{\alpha}$ is the Chern-Weil map (see section 1.5), determined by the gauge potentials of a connection. So $\left\{c w_{\alpha}(\omega)\right\}_{\alpha}$ is a Cech 0 -cochain with values in $p$-forms on the open subsets, $p$ is the degree of $\omega$. Then $c_{\phi}$ is a chain map: just check that $\left.c_{\phi}\right|_{W(\mathfrak{g})}$ commutes with the differentials. But $c w_{\alpha}$ is a homomorphism of DG algebras, so $d_{U_{\alpha}} c w_{\alpha}(\omega)=c w_{\alpha}(\partial \omega)$, and $\delta\left(\left\{c w_{\alpha}(\omega)\right\}_{\alpha}\right)=\left\{c w_{\alpha}(\omega)-c w_{\beta}(\omega)\right\}_{\alpha \prec \beta}$, but from the formulas (2.13), (2.14), the Bianchi identity (1.11) and the definition of $c w_{\alpha}$ it follows that

$$
c w_{\alpha}\left(\omega^{(1)}\right) \wedge g_{\alpha \beta}^{*}\left(\omega^{(2)}\right)=c w_{\beta}(\omega),
$$

here $\tilde{\Delta}_{W}^{*}(\omega)=\omega^{(1)} \otimes \omega^{(2)}$. So $\delta\left(\left\{c w_{\alpha}(\omega)\right\}_{\alpha}\right)=c_{\phi}\left(\tilde{\Delta}_{W}^{*}(\omega)\right)$.

Thus $c_{\phi}: F(W) \rightarrow \check{C}\left(\mathcal{U}, \Omega_{D R}(U)\right)$ is a chain map. The composition of $c_{\phi}$ with (2.15) coincides with $\tilde{\phi}$ and the composition of $c_{\phi}$ with (2.16) coincides with the Chern-Weil homomorphism, composed with the evident map from $\Omega_{D R}(X)$ to Čech complex. Since the inclusions (2.15) and (2.16) are homotopic to each other, we conclude that for any basic element in Weil complex $x \in W(\mathfrak{g})^{\text {bas }}$, there exists a corresponding element $y \in F\left(\Omega_{G}\right)$, 
which gives the same class in $F(W)$. Thus there exists an element $z \in F(W)$, such that $d z=i_{1}(x)-i_{2}(y)$. Applying $c_{\phi}$ to both sides of this equality we conclude that for every closed form $\xi \in \Omega_{D R}^{p}(X)$ in the image of the ChernWeil homomorphism, we can find a cocycle $\eta \in \sum_{i+j=p} \check{C}^{i}\left(\mathcal{U}, \Omega_{D R}^{j}(U)\right)$ in the image of $\tilde{\phi}$ and a cochain $\zeta \in \sum_{i+j=p-1} \check{C}^{i}\left(\mathcal{U}, \Omega_{D R}^{j}(U)\right)$ such that

$$
\xi-\eta=(d+\delta) \zeta
$$

Remark 2.5. - One can change the proof of 2.4 so that it would give explicit formulas for the cocycles $\alpha \in F\left(\Omega_{G}\right)$ cohomologuous to elements in $W(\mathfrak{g})^{\text {bas }}$. Applying $\tilde{\phi}$ to these cocycles, one obtains expressions for the Chern classes in terms of the cocycle $g_{\alpha \beta}$. This procedure can be rendered completely algorithmic with the help of the perturbation lemma, see the section 1.2. A reader, interested in details and concrete formulas can refer to [23]. There are other approaches that give explicit formulas for the characteristic classes of a principal bundle in the terms of its transition cocycle $\left\{g_{\alpha \beta}\right\}$. For instance see the last chapter of book [3] or [10]. This construction is closely related to the one, we use here, which is also explained in [23].

\subsection{Another geometric construction of twisting cochain associated with principal bundles}

There exist many ways to write down a twisting cochain, corresponding to a princiapl bundle; e.g. using gauge transformations, one can vary a given one. This subsection is devoted to a discussion of a particular way to deform the twisting cochain we constructed in section 2.1. We shall first describe this deformation as a variant of the twisting cochain associated with a principal bundle and then explain how it is related to $\phi_{P}$.

Let $\mathcal{U}=\left\{U_{i}\right\}$ be the trivializing open cover of the base $X$ of the principal bundle $P$ with structure group $G$. Let $\omega \in \Omega_{D R}(P) \otimes \mathfrak{g}$ be a connection 1form on $P$ and $A_{\alpha}=\sum_{k} A_{\alpha}^{k} \otimes X_{k}$ the corresponding local gauge potentials, where $X_{k}$ is a basis of $\mathfrak{g}$ and $A_{\alpha}^{k}$ are 1-forms on $U_{\alpha}$. Let $F_{\alpha}=\sum_{k} F_{\alpha}^{k} \otimes X_{k}$ be the local curvature forms, corresponding to $A_{\alpha}$. Consider the following map $\xi: \Omega_{G} \rightarrow \check{C}\left(\mathcal{U}, \Omega_{D R}(U)\right)$ (summation is taken over repeating indices):

$$
\xi(x)=\left\{A_{\alpha}^{k} X_{k}(x)(e)-F_{\alpha}^{k} I_{k}(x)(e)\right\}_{\alpha}+\left\{1_{\alpha \beta} x(e)-x^{0}\left(g_{\alpha \beta}\right)\right\}_{\alpha \prec \beta} .
$$

Here $e$ is the unit of $G$, so that $\epsilon: x \mapsto x^{0}(e)=\epsilon(x)$ is the counit of $\Omega_{G}$, $x^{0}$ denotes the projection of $x$ to the space of 0 -degree forms; $I_{k}$ is the contraction of a form $x \in \Omega_{G}$ with the left-invariant vector field, generated by $X_{k}$ and we regard $X_{k}$ at the same time as the Lie derivative. Observe that $\xi(x)=0$ for all $x, \operatorname{deg} x \geqslant 1$. 
Let us check, that $\xi(x)$ is a twisting cochain. To check the equation (1.3) for $\xi$ it is enough to take $x$ of degrees not greater than 2: $\xi(x)=0$ for $\operatorname{deg} x>1$, the same is true for $d \xi$, and $\xi \cup \xi$ vanish for $\operatorname{deg} x \geqslant 2$. So, if $\operatorname{deg} x=0$, we get (in this and succeeding formulas we omit the restriction signs)

$$
\begin{aligned}
d(\xi)(x)= & (d+\delta) \xi(x)-\xi(d x)=\left\{C_{i j}^{k} A_{\alpha}^{i} \wedge A_{\alpha}^{j} X_{k}(x)(e)\right\}_{\alpha} \\
& +\left\{A_{\beta}^{k} X_{k}(x)(e)-A_{\alpha}^{k} X_{k}(x)(e)-d x\left(g_{\alpha \beta}\right)\right\}_{\alpha \prec \beta} \\
+ & \left\{1_{\alpha \beta \gamma} x(e)-x\left(g_{\beta \gamma}\right)+x\left(g_{\alpha \gamma}\right)-x\left(g_{\alpha \beta}\right)\right\}_{\alpha \prec \beta \prec \gamma .}
\end{aligned}
$$

Here we used the Cartan identity $X_{k}=\left[I_{k}, d\right]$ and the Bianchi identity. Similarly, from the definition of coproduct in $\mathcal{A}(G)$, it follows that $x^{(1)}(g) x^{(2)}(h)=x(g h)$ for all $x \in \mathcal{A}(G)$ and all $g, h \in G$. From this equality we obtain

$$
\begin{aligned}
X\left(x^{(1)}\right)(e) x^{(2)}(g) & =\left.\frac{d}{d t}\right|_{t=0} x^{(1)}(\exp (t X)) x^{(2)}(g) \\
& =\left.\frac{d}{d t}\right|_{t=0} x(\exp (t X) g)=X(x)(g),
\end{aligned}
$$

for all $X \in \mathfrak{g}$. Similarly, $x^{(1)}(g) X\left(x^{(2)}\right)(e)=A d_{g} X(x)(g)$ and

$$
X_{i} \cup X_{j}(x)(e)=X_{i}\left(x^{(1)}\right)(e) X_{j}\left(x^{(2)}\right)(e)=\left[X_{i}, X_{j}\right](x)(e) .
$$

Thus we compute (we use the sign rule from (2.4)):

$$
\begin{aligned}
& \xi \cup \xi(x)=\left\{C_{i j}^{k} A_{\alpha}^{i} \wedge A_{\alpha}^{j} X_{k}(x)(e)\right\}_{\alpha} \\
& +\left\{-A_{\alpha}^{k} X_{k}(x)(e)+A_{\alpha}^{k} X_{k}(x)\left(g_{\alpha \beta}\right)+A_{\beta}^{k} X_{k}(x)(e)-A_{\beta}^{k} A d_{g_{\alpha \beta}} X_{k}(x)\left(g_{\alpha \beta}\right)\right\}_{\alpha \prec \beta} \\
& +\left\{1_{\alpha \beta \gamma} x(e)-x\left(g_{\beta \gamma}\right)+x\left(g_{\alpha \gamma}\right)-x\left(g_{\alpha \beta}\right)\right\}_{\alpha \prec \beta \prec \gamma} .
\end{aligned}
$$

The difference of these two expressions is equal to

$$
d(\xi)(x)-\xi \cup \xi(x)=\left\{A_{\beta}^{k} A d_{g_{\alpha \beta}} X_{k}(x)\left(g_{\alpha \beta}\right)-A_{\alpha}^{k} X_{k}(x)\left(g_{\alpha \beta}\right)-d x\left(g_{\alpha \beta}\right)\right\}_{\alpha \prec \beta} .
$$

But since $g_{\alpha \beta} A_{\beta}=A_{\alpha} g_{\alpha \beta}+d g_{\alpha \beta}$ (see (1.11)), this expression is equal to 0 . Similarly, if $\operatorname{deg} x=1$, we have (we use the second Bianchi identity):

$$
d(\xi)(x)=\left\{-C_{i j}^{k} F_{\alpha}^{i} \wedge A_{\alpha}^{j} I_{k}(x)(e)\right\}_{\alpha}+\left\{F_{\beta}^{k} I_{k}(x)(e)-F_{\alpha}^{k} I_{k}(x)(e)\right\}_{\alpha \prec \beta} .
$$

But from the definition of coproduct of de Rham forms on a group, it follows that

$$
X_{i} \cup I_{j}(x)(e)-I_{j} \cup X_{i}(x)(e)=\left[X_{i}, I_{j}\right](x)(e)=C_{i j}^{k} I_{k}(x)(e),
$$


so using the identities, similar to the considered above, we get the following formula:

$$
\xi \cup \xi(x)=\left\{-C_{i j}^{k} F_{\alpha}^{i} \wedge A_{\alpha}^{j} I_{k}(x)(e)\right\}_{\alpha}+\left\{F_{\beta}^{k} I_{k}(x)(e)-F_{\alpha}^{k} I_{k}(x)(e)\right\}_{\alpha \prec \beta},
$$

(we used the equation (1.14): $g_{\alpha \beta} F_{\beta}=F_{\alpha} g_{\alpha \beta}$ ). Subtracting this formula from the previous one, we get 0 . Finally, if $\operatorname{deg} x=2$, then $d(\xi)(x)=0$, and

$$
\xi \cup \xi(x)=\left\{F_{\alpha}^{i} I_{i}\left(x^{(1)}\right)(e) \wedge F_{\alpha}^{j} I_{j}\left(x^{(2)}\right)(e)\right\}_{\alpha} .
$$

But since

$$
I_{i}\left(x^{(1)}\right)(e) I_{j}\left(x^{(2)}\right)(e)+I_{j}\left(x^{(1)}\right)(e) I_{i}\left(x^{(2)}\right)(e)=\left[I_{i}, I_{j}\right](x)(e)=0,
$$

this is equal to 0 . Thus $\xi$ is a twisting cochain. One can show that the twisted tensor product, associate with $\xi$ is homotopic to the cochain complex of the principal bundle. Then from the general theory it follows that $\xi$ should be equivalent to $\phi_{P}$, see section 2.1. We shall prove this equivalence directly by constructing the corresponding gauge transformation. To this end, consider the following formula: $x \stackrel{c}{\mapsto}\left\{\exp \left(\sum_{k} A_{\alpha}^{k} I_{k}\right)(x)(e)\right\}_{\alpha}, c: \Omega_{G} \rightarrow$ $\check{C}\left(\mathcal{U}, \Omega_{D R}(U)\right)$. This map is invertible with respect to convolution, thus it induces a gauge transformation, such that

$$
\begin{aligned}
\left(d(c)(x)+\left(c \cup \phi_{P}\right)(x)\right)^{0} & =(d(c)(x))^{0}=\left\{A_{\alpha}^{i} X_{i}(x)(e)-F_{\alpha}^{i} I_{i}(x)(e)\right\}_{\alpha} \\
& =((\xi \cup c)(x))^{0},
\end{aligned}
$$

$\left(()^{k}\right.$ denotes the $k$-Čech degree part of a cochain). Indeed since both sides of this equality are derivations in $x$, commuting with $d$ and are represented by sums of finite number of terms that lie in $\Omega_{D R}\left(U_{\alpha}\right)$, it is enough to check this equality only for $\operatorname{deg} x=0$, and only on local level, which is easy. Further, since $\left(\phi_{P}(x)\right)^{k}=(\xi(x))^{k}=0, k \geqslant 2$ and $(c(x))^{k}=0, k \geqslant 1$, we should compare only the 1-Cech degree part of the corresponding equality. Once again, it is enough to do it for $\operatorname{deg} x=0$, which is simple.

Observe that the equivalence of $\xi$ and $\phi_{P}$ means that the class of $\xi$ modulo the gauge transformations does not depend on the choice of connection. One can also use this result to find the homotopies between the expressions representing the characteristic classes of $P$ in terms of a connection and its curvature and the expressions, involving the cocycle $g_{\alpha \beta}$ : just apply the universal construction of the chain homotopy between the characteristic maps $\tilde{\phi}_{P}$ and $\tilde{\xi}$ induced by a gauge transformation and keep in mind that only the terms with $F_{\alpha}$ in $\tilde{\xi}$ will contribute to the image of $x \in \Omega_{D R}(G)$, when $\operatorname{deg} x>0$. 
Remark 2.6. - One can get more insight of relation between the twisting cochains $\phi_{P}$ and $\xi$ as follows. Let $\widetilde{\Omega_{G}}$ be the algebra bundle over $X$ with fibre $\Omega_{G}$, associated with $P$ (we let $G$ act on $\Omega_{G}$ by left translations). Let $\Gamma\left(\widetilde{\Omega_{G}}\right)$ denote the space of differential forms on $X$ with values in this bundle. Consider the map $\tilde{c}: \Gamma\left(\widetilde{\Omega_{G}}\right) \rightarrow \Omega_{D R}(P)$, given by the local formula:

$$
f_{\alpha} \omega^{i_{1}} \wedge \ldots \wedge \omega^{i_{p}} \otimes h_{\alpha}(x) \mapsto f_{\alpha} \exp \left(\sum_{i} I_{i} \otimes A_{\alpha}^{i}\right)\left(\omega^{i_{1}} \wedge \ldots \wedge \omega^{i_{p}}\right) \otimes h_{\alpha}(x) .
$$

Here $x \in X$ and $h_{\alpha}(x)$ is a differential form on $U_{\alpha}$. This map is welldefined, i.e. it doesn't depend on the choice of trivialization. Moreover, it is an isomorphism of vector spaces (it is enough to prove this locally, which is evident - its inverse is given by $\left.\exp \left(-\sum_{i} I_{i} \otimes A_{\alpha}^{i}\right)\right)$. The map $\tilde{c}$ intertwines the de Rham differential on $P$ with a covariant derivative on the complex of sections $\Gamma\left(\widetilde{\Omega_{G}}\right)$. Now the twisting cochain $\xi$ is just the result of the construction similar to that of $\phi_{P}$ applied to $\Gamma\left(\widetilde{\Omega_{G}}\right)$ equipped with the differential, induced from $\Omega_{D R}(P)$.

The twisting cochain $\xi$ has one important advantage: one can restrict it to the subspace of right-invariant differential forms on $G$, which is isomorphic to the Chevalley-Eilenberg complex $C^{*}(\mathfrak{g})$ of the Lie algebra of $G$. Observe, that $\mathfrak{g}$ acts on $C^{*}(\mathfrak{g})$ by Lie derivatives $X, X \in \mathfrak{g}$. Besides this there are also convolutions (inner multiplications) with elements of $\mathfrak{g}$. Thus we obtain the collection of graded derivatives $X, I_{X}$, verifying the usual Cartan identities. Since for all $p, C^{p}(\mathfrak{g})$ is a finite-dimensional vector space, the map

$$
G \times C^{p}(\mathfrak{g}) \rightarrow C^{p}(\mathfrak{g}),(g, c) \mapsto c^{g},
$$

where $c^{g}\left(X_{1}, \ldots, X_{p}\right)=c\left(A d_{g}\left(X_{1}\right), \ldots, A d_{g}\left(X_{p}\right)\right)$, can be regarded dually as a map

$$
C^{p}(\mathfrak{g}) \rightarrow C^{p}(\mathfrak{g}) \otimes \mathcal{A}(G) .
$$

Thus, the following formula gives a twisting map in the sense of section 1.3, defined on the Chevalley complex $C^{*}(\mathfrak{g})$, associated with $P$ and taking values in $\check{C}^{*}\left(\mathcal{U}, \Omega_{D R}(U)\right)$ :

$$
\begin{aligned}
& \psi_{P}: C^{*}(\mathfrak{g}) \rightarrow \check{C}^{*}\left(\mathcal{U}, \Omega_{D R}(U)\right), \\
& \psi_{P}(c)=\left\{\sum_{i}\left\{A_{i} \otimes X^{i} c-F_{i} \otimes I_{X^{i}} c\right\}\right\}_{\alpha}+\left\{1_{\alpha \beta} c(0)-c^{g_{\alpha \beta}}\right\}_{\alpha \prec \beta} .
\end{aligned}
$$

\subsection{From Čech complex to de Rham complex}

In this section we shall describe a construction, that allows one pass from twisting cochains and twisting maps with values in Čech complexes, 
to twisting cochains with values in the algebra of de Rham forms on the base. This construction depends on the choice of the partition of unity on the base, and is not quite canonical. Therefore we shall also give another construction of this sort, based on the notion of realisation of a cosimplicial algebra.

First of all, there is an evident chain map $i^{\prime}: \Omega_{D R}(X) \rightarrow \check{C}\left(\mathcal{U}, \Omega_{D R}(U)\right)$, given by

$$
h \stackrel{i^{\prime}}{\mapsto}\left\{h_{\left.\right|_{U_{\alpha}}}\right\}_{\alpha} .
$$

In previous sections we called this map "localization" of a differential form. It is a homomorphism of algebras. Moreover, this map establishes an isomorphism on the level of cohomology. Thus $i^{\prime}$ is a quasi-isomorphism of DG algebras and hence there exists a homotopy inverse $A_{\infty}$-map $\mathcal{P}: \check{C}\left(\mathcal{U}, \Omega_{D R}(U)\right)$ $\rightarrow \Omega_{D R}(X)$, see section 1.2. In addition, in section 1.3 we explained how one can use $\mathcal{P}$ to define a $\Omega_{D R}(X)$-valued twisting cochain on $\Omega_{G}$. Our purpose is to describe the perturbation data that allows one to obtain the explicit formulas.

We need to find the higher maps that constitute the $A_{\infty}$ homotopy inverse to $i^{\prime}$. In general, this would demand solving inductively all the equations, that define $\mathcal{P}(n)$. However, we prefer to use the perturbation approach, explained in section 1.2. Let us first define a chain map $p: \check{C}\left(\mathcal{U}, \Omega_{D R}(U)\right) \rightarrow \Omega_{D R}(X)$, homotopy inverse to $i^{\prime} ; p$ need not commute with multiplication, we only want it commute with differentials. So we regard $\check{C}\left(\mathcal{U}, \Omega_{D R}(U)\right)$ as a bicomplex with two differentials, $\delta$ and $d$, (Čech and de Rham differentials respectively). Forget about $d$ for a while, that is consider $\Omega_{D R}(X)$ as a complex with zero differential. Then the map $i_{0}=i^{\prime}$ still induces an isomorphism in cohomology, but this time it is possible to give an explicit inverse map: let $\left\{\varphi_{\alpha}\right\}$ be a partition of unity on $X$ associated with $\mathcal{U}$. Then consider the map

$$
\begin{gathered}
p_{0}: \check{C}\left(\mathcal{U}, \Omega_{D R}(U)\right) \rightarrow \Omega_{D R}(X), \\
p_{0}\left(\left\{h_{\alpha_{1} \ldots \alpha_{n}}\right\}_{\alpha_{1} \prec \ldots \prec \alpha_{n}}\right)=\left\{\begin{array}{l}
0, n \geqslant 2, \\
\sum_{\alpha} h_{\alpha} \varphi_{\alpha}, n=1 .
\end{array}\right.
\end{gathered}
$$

It is easy to check that $p_{0} \circ i_{0}=I d$ and hence $p_{0}$ is a homotopy inverse to $i_{0}$. Moreover, it is possible to write down an explicit formula for the chain homotopy. To this end we extend a Čech cochain $\left\{h_{\alpha_{1} \ldots \alpha_{n}}\right\}_{\alpha_{1} \prec \ldots \prec \alpha_{n}}$ to an arbitrary (i.e. not necessarilly ordered) collection of $n$ indices $\alpha_{1}, \ldots \alpha_{n}$ so that $h_{\alpha_{\sigma(1)} \ldots \alpha_{\sigma(n)}}=(-1)^{\sigma} h_{\alpha_{1} \ldots \alpha_{n}}, A$ will denote the anti-symmetrization procedure, $S$ its inverse. Clearly, there is a 1-1 correspondence between the antisymmetrized Čech cochains and the usual (ordered) Čech cochains. Then 
the Čech differential $\delta$ can be extended to the space of anti-symmetrized cochains by the same formula:

$$
\delta\left(\left\{h_{\alpha_{1} \ldots \alpha_{n}}\right\}\right)_{\alpha_{1} \ldots \alpha_{n} \alpha_{n+1}}=\sum_{i}(-1)^{i} h_{\alpha_{1} \ldots \alpha_{i} \ldots \alpha_{n+1}} .
$$

It is easy to see that $\delta$ commutes with the anti-symmetrization, i.e. that $\delta A(h)=A(\delta h)$. Let $S$ denote the inverse of $A$. Having this in mind, let us define the following map:

$$
\begin{gathered}
H_{0}: \check{C}^{*}\left(\mathcal{U}, \Omega_{D R}(U)\right) \rightarrow \check{C}^{*-1}\left(\mathcal{U}, \Omega_{D R}(U)\right), \\
H_{0}\left(\left\{h_{\alpha_{1} \ldots \alpha_{n}}\right\}\right)_{\alpha_{1} \prec \ldots \prec \alpha_{n-1}}=S\left(\sum_{\alpha_{n}} A\left(\left\{h_{\alpha_{1} \ldots \alpha_{n}}\right\}\right) \varphi_{\alpha_{n}}\right) .
\end{gathered}
$$

In a more explicit way, this formula can be written as follows

$$
\begin{aligned}
H_{0}\left(\left\{h_{\alpha_{1} \ldots \alpha_{n}}\right\}\right)_{\alpha_{1} \prec \ldots \prec \alpha_{n-1}} & \\
& =\sum_{i=1}^{n-1}(-1)^{i-1}\left\{\sum_{\alpha_{i-1} \prec \alpha \prec \alpha_{i}} h_{\alpha_{1} \ldots \alpha_{i-1} \alpha \alpha_{i} \ldots \alpha_{n}} \varphi_{\alpha}\right\} \\
& +(-1)^{n-1} \sum_{\alpha_{n-1} \prec \alpha} h_{\alpha_{1} \ldots \alpha_{n-1}} \varphi_{\alpha} .
\end{aligned}
$$

Then the following equations can be easily checked:

$$
H_{0} H_{0}=0, \quad H_{0} i_{0}=0, \quad p_{0} H_{0}=0, \quad i_{0} p_{0}-I d=\delta H_{0}+H_{0} \delta
$$

(compare with (1.1)). Taking into consideration these equations and the equality $p_{0} \circ i_{0}=I d$, we observe that we are now in the situation, described by the "perturbation lemma", see [22]. This lemma ensures that for any additional differential $d$ on $\breve{C}\left(\mathcal{U}, \Omega_{D R}(U)\right)$, one can find a differential $d^{\prime}$ on $\Omega_{D R}(X)$, and maps $i, p, H$ with the same domains and ranges as $i_{0}, p_{0}, H_{0}$, which will verify the same equations $(2.20)$ but with respect to the differential $d+\delta$ on $\check{C}\left(\mathcal{U}, \Omega_{D R}(U)\right)$ and the differential $d^{\prime}=d^{\prime}+0$ on $\Omega_{D R}(X)$ (if there were a nonzero differential $\delta^{\prime}$ on $\Omega_{D R}(X)$ before perturbation, we would use $d^{\prime}+\delta^{\prime}$ here). These maps are given by the formulas

$$
\begin{aligned}
& d^{\prime}=p_{0} d i_{0}+p_{0} d H_{0} d i_{0}+p_{0} d H_{0} d H_{0} d i_{0} \ldots, \\
& i=i_{0}+H_{0} d i_{0}+H_{0} d H_{0} d i_{0}+\ldots, \\
& p=p_{0}+p_{0} d H_{0}+p_{0} d H_{0} d H_{0}+\ldots \\
& H=H_{0}+H_{0} d H_{0}+H_{0} d H_{0} d H_{0}+\ldots
\end{aligned}
$$

In our case, $i_{0}=i^{\prime}$ commutes with the de Rham differentials $d$ on $\Omega_{D R}(X)$ and $\check{C}\left(\mathcal{U}, \Omega_{D R}(U)\right)$, so $d^{\prime}=d$ and $i=i_{0}=i^{\prime}$. Thus $p$ is the homotopy inverse map for $i^{\prime}$ we were looking for. 
Now we have enough the data to determine the $A_{\infty}$-inverse of the "localization" $i^{\prime}=i$ : the map $i_{\infty}^{-1}$ and the corresponding homotopy are given by equations (1.2). One should take $f=i$ and $f^{-1}=p$ and use the multiplication in $\check{C}\left(\mathcal{U}, \Omega_{D R}(U)\right)=A_{1}$. The formulas we obtain here are not quite canonical, they depend on the partition of unity and it is not always evident, how to find it. Therefore we suggest here another construction, based on the simplicial constructions.

Let $\mathcal{U}=\left\{U_{1}, \ldots, U_{N}\right\}$ be a finite (ordered) open cover of a topological space $X$. The order is given by the indices $1, \ldots, N$ and wherever we have a string $\ldots, U_{\alpha}, U_{\beta}, U_{\gamma}, \ldots$ of elements of $\mathcal{U}$, we shall assume that the order is not violated, i.e. .. $\preceq \alpha \preceq \beta \preceq \gamma \preceq \ldots$. Consider the following simplicial topological space $\mathcal{N} \mathcal{U}_{*}$ :

$$
\mathcal{N} \mathcal{U}_{n}=\coprod_{\left\{U_{0}, \ldots, U_{n}\right\}, U_{i} \in \mathcal{U}} U_{0} \cap \ldots \cap U_{n} .
$$

The face and degeneracy maps in $\mathcal{N U}_{*}$ are given by the formulas

$$
\partial_{p}(x)=i_{p}(x), \quad s_{q}(x)=j_{q}(x),
$$

$p, q=0, \ldots, n$, where

$$
i_{p}: U_{0} \cap \ldots \cap U_{p} \cap \ldots \cap U_{n} \rightarrow U_{0} \cap \ldots \cap \widehat{U}_{p} \cap \ldots \cap U_{n}
$$

is the natural inclusion and

$j_{p}: U_{0} \cap \ldots \cap U_{p} \cap \ldots \cap U_{n} \rightarrow U_{0} \cap \ldots \cap U_{p} \cap U_{p} \cap \ldots \cap U_{n}=U_{0} \cap \ldots \cap U_{p} \cap \ldots \cap U_{n}$

is the identity map. The simplicial relations are readily verified.

We shall call the topological space $\mathcal{N U}_{*}$ the topological nerve of $\mathcal{U}$. The adjective "topological" is used to distinguish it from the usual nerve of an open cover of a space, which we shall call combinatoric. Observe, that $\mathcal{N} \mathcal{U}_{*}$ is in effect a simplicial manifold, if $X$ is a manifold. Also observe that if $\mathcal{V}$ is a finer open cover, i.e. if for every $V_{k} \in \mathcal{V}$ there exists an element $U_{j} \in \mathcal{U}$, such that $V_{k} \subseteq U_{j}$, then there is a simplicial map $\mathcal{N} \mathcal{V}_{*} \rightarrow \mathcal{N} \mathcal{U}_{*}$ : choose $U_{j}$ with described property for every $V_{k} \in \mathcal{V}$ and extend the inclusion maps to all the levels of $\mathcal{N} \mathcal{V}_{*}$ in a natural way. In particular, if $\mathcal{X}$ is the open cover, consisting of the only one open set $U=X$, then $\mathcal{N} \mathcal{X}_{*}$ is the constant simplicial space, $\mathcal{N} \mathcal{X}_{n}=X, n \geqslant 0$ and all the structure maps are equal to identity. Hence there exists a simplicial map $(Q \mathcal{U})_{*}: \mathcal{N U}_{*} \rightarrow \mathcal{N} \mathcal{X}_{*}=X$ for every open cover $\mathcal{U}$.

Proposition 2.7. - For any finite open cover $\mathcal{U}$ of $X, Q_{\mathcal{U}}:\left|\mathcal{N U}_{*}\right| \rightarrow$ $X$ is homotopy equivalence. 
Proof. - is by induction in the number of elements in $\mathcal{U}$. The base of induction $N=1$ is clear. Let $N=2$, so $\mathcal{U}=\left\{U_{1}, U_{2}\right\}, X=U_{1} \cup U_{2}$. Let $X_{1}=U_{1}, X_{2}=U_{2}$ and $X_{12}=U_{1} \cap U_{2}$. The open cover $\mathcal{U}$ of $X$ generates open covers on $X_{1}, X_{2}$ and $X_{12}: \mathcal{U}_{1}=\left\{U_{1}\right\}, \mathcal{U}_{2}=\left\{U_{2}\right\}$ and $\mathcal{U}_{12}=\left\{U_{1} \cap U_{2}\right\}$. We have the following diagram

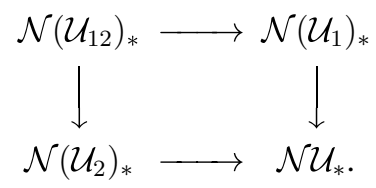

This square is cocartesian in the category of simplicial spaces. The geometric realization functor applied to (2.22) gives a homotopy cocartesian square in topological category. Maps $Q_{\mathcal{U}_{12}}, Q_{\mathcal{U}_{1}}, Q_{\mathcal{U}_{2}}$ and $Q_{\mathcal{U}}$ commute with (2.22) and send it to the cocartesian square of spaces

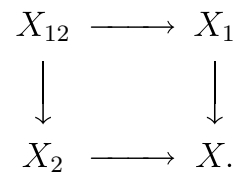

But as we know, the maps $Q_{\mathcal{U}_{12}}, Q_{\mathcal{U}_{1}}$ and $Q_{\mathcal{U}_{2}}$ are homotopy equivalences, hence the map $Q_{\mathcal{N}}$ is a homotopy equivalence. The general case is treated in a similar way: if the cover $\mathcal{U}$ consists of $N$ elements, $\mathcal{U}=\left\{U_{1}, \ldots, U_{N}\right\}$, we put $X_{1}=U_{1} \cup \ldots \cup U_{N-1}, X_{2}=U_{N}$ and $X_{12}=X_{1} \cap X_{2}$ and consider the square similar to $(2.22)$.

Consider now a simplicial manifold $X_{*}$. Apply the contravariant functor of de Rham differential forms to $X_{*}$ degree-wise. Let us define the realisation of the cosimplicial DG algebra $\Omega_{D R}\left(X_{*}\right)$ in the category of commutative DG algebras: $\left|\Omega_{D R}\left(X_{*}\right)\right|$ should be a commutative DG algebra, which will play the role of de Rham forms on the realisation of $X_{*}$. To this end we consider first the algebraic building blocks, similar to the geometric simplices $\triangle^{i}$ of geometric realisation. They should be DG algebras, modeling the de Rham forms on simplices. This can be done directly by putting $\Omega_{i}=\Omega_{D R}\left(\triangle^{i}\right)$, but we shall prefer a more delicate approach, due to Sullivan, Thom and others, and use the polynomial differential forms on simplices. So we put

$$
\Omega_{n}=\mathbb{R}\left[t_{0}, \ldots, t_{n} ; d t_{0}, \ldots, d t_{n}\right] /\left(t_{0}+t_{1}+\ldots+t_{n}=1\right),
$$

where $\mathbb{R}\left[t_{0}, \ldots, t_{n} ; d t_{0}, \ldots, d t_{n}\right]$ is the free graded-commutative algebra, generated by $t_{i}, d t_{i}, \operatorname{deg} t_{i}=0, \operatorname{deg} d t_{i}=1, i=0, \ldots, n$ with differential $d$, induced by $d\left(t_{i}\right)=d t_{i}$, which we quotient by the differential ideal, generated by the relation $t_{0}+\ldots+t_{n}=1$ (e.g. $d t_{0}+\ldots+d t_{n}=0$, etc.). Thus 
we obtain the the collection of DG algebras $\left\{\Omega_{n}(\triangle)\right\}_{n \geqslant 0}$. We introduce the structure of simplicial DG algebra on it by the formulae

$$
\partial_{k}\left(t_{i}\right)=\left\{\begin{array}{ll}
t_{i}, & i<k, \\
0, & i=k, \\
t_{i-1}, & i>k ;
\end{array} \quad s_{j}\left(t_{i}\right)= \begin{cases}t_{i}, & i<j, \\
t_{i}+t_{i+1}, & i=j, \\
t_{i+1}, & i>j .\end{cases}\right.
$$

The usual simplicial relations are easily checked. One can regard this simplicial differential algebra as the algebra of polynomial differential forms on geometric simplices.

Our purpose is to dualise the definition of the realization of a simplicial space, so we take the following definition of the realisation of a cosimplicial algebra (compare the definition of $\left|X_{*}\right|$ from section 1.4):

DEFINITION 2.8. - The realization of a cosimplicial algebra $\left\{\Omega^{i}\right\}$ is the commutative DG algebra $\left|\Omega^{*}\right|$ below:

$$
\left|\Omega^{*}\right|=\left\{\left\{\omega_{i} \otimes \varphi_{i}\right\} \in \prod_{i=0}^{\infty} \Omega^{i} \otimes \Omega_{i}(\triangle) \mid \theta^{*}\left(\omega_{i}\right) \otimes \varphi_{i}=\omega_{j} \otimes \theta_{*}\left(\varphi_{j}\right)\right\} .
$$

Here $\theta: \mathbf{i} \rightarrow \mathbf{j}$ is a morphism in simplicial category, and $\theta_{*}, \theta^{*}$ the corresponding simplicial and cosimplicial structure maps in $\Omega_{*}(\triangle)$ and $\Omega^{*}$. Since maps $\theta_{*}$ and $\theta^{*}$ are homomorphisms of algebras, $\left|\Omega^{*}\right|$ is a subalgebra of the direct product of algebras.

Suppose now that $\left\{\Omega^{*}\right\}$ is equal to $\left\{\Omega_{D R}\left(\mathcal{N} \mathcal{U}_{i}\right)\right\}$ where $\mathcal{U}$ is an open cover of a smooth manifold $X$. Then there exists an evident map $Q_{\mathcal{U}}^{*}$ : $\Omega_{D R}(X) \rightarrow\left|\Omega_{D R}\left(\mathcal{N U}_{*}\right)\right|$, given by

$$
\omega \mapsto\left\{\left(\bigoplus_{i} \omega_{\left.\right|_{U_{i}}}\right) \otimes 1,\left(\bigoplus_{i \leqslant j} \omega_{\left.\right|_{U_{i} \cap U_{j}}}\right) \otimes 1, \ldots\right\} \in \prod_{i=0}^{\infty} \Omega_{D R}\left(\mathcal{N U}_{i}\right) \otimes \Omega_{i}(\triangle) .
$$

Here $\mathcal{U}=\left\{U_{1}, \ldots, U_{N}\right\}, 1 \in \Omega_{0}(\triangle)$ is the unit, and the projection of $Q_{\mathcal{U}}^{*}(\omega)$ to $\Omega_{D R}\left(\mathcal{N U}_{k}\right) \otimes \Omega_{k}(\triangle)$ is equal to the direct sum of restrictions of $\omega$ to all intersections $U_{i_{0}} \cap \ldots \cap U_{i_{k}}$ for all nondecreasing sequences of indices $i_{0} \leqslant \ldots \leqslant i_{k}$, tensored by the identity in $\Omega_{k}(\triangle)$. Then the image of $Q_{\mathcal{U}}^{*}$ belongs to $\left|\Omega_{D R}\left(\mathcal{N U}_{*}\right)\right|$ : it is enough to show that $\delta_{p}\left(Q_{\mathcal{U}}^{*}(\omega)_{k}\right)=$ $Q_{\mathcal{U}}^{*}(\omega)_{k+1}$ and $\sigma_{q}\left(Q_{\mathcal{U}}^{*}(\omega)_{k}\right)=Q_{\mathcal{U}}^{*}(\omega)_{k-1}$ for all $k \geqslant 0, p=0, \ldots, k+1$ and $q=0, \ldots, k-1$. Here $Q_{\mathcal{U}}^{*}(\omega)_{k}$ denotes the projection of $Q_{\mathcal{U}}^{*}(\omega)$ onto $\Omega_{D R}\left(\mathcal{N U}_{k}\right) \otimes \Omega_{k}(\triangle)$. But $\delta_{p}=\left(\partial_{p}\right)^{*}=i_{p}^{*}$ and

$$
i_{p}^{*}\left(\omega_{\left.\right|_{U_{0} \cap \ldots \cap \widehat{U}_{p} \cap \ldots \cap U_{k+1}}}\right)=\omega_{\left.\right|_{U_{0} \cap \ldots \cap U_{k+1}}} .
$$


Similarly, $\sigma_{q}=\left(s_{q}\right)^{*}=j_{q}^{*}$ and we get the result. Observe, that since the restriction of differential forms is a homomorphism of algebras, $Q_{\mathcal{U}}^{*}$ is a homomorphism of algebras. The following proposition is an algebraic analogue of 2.7 .

Proposition 2.9. - For any finite open cover $\mathcal{U}$ of a manifold $X$, the map $Q_{\mathcal{N}}^{*}$ is a quasi-isomorphism of $D G$ algebras, $Q_{\mathcal{N}}^{*}: \Omega_{D R}(B) \rightarrow$ $\left|\Omega_{D R}\left(\mathcal{N U}_{*}\right)\right|$.

Proof. - Use the Meier-Vietoris exact sequence and the 5-lemma.

Let $\mathcal{U}$ be an open cover of $X$, trivializing the principal bundle $P \stackrel{G}{\rightarrow}$ $X$. We shall construct a twisting cochain with values in the realization $\left|\Omega_{D R}\left(\mathcal{N} \mathcal{U}_{*}\right)\right|$ of $\Omega_{D R}\left(\mathcal{N} \mathcal{U}_{*}\right)$ : we are going to apply the $A_{\infty}$ map constructions like we did it before. First, let us compare the Cech complex and the algebra $\left|\Omega_{D R}\left(\mathcal{N U}_{*}\right)\right|$. They cannot be isomorphic, since the former one is a noncommutative while the latter one is a graded commutative. However, we have the following result

Proposition 2.10. - The algebra $\left|\Omega_{D R}\left(\mathcal{N U}_{*}\right)\right|$ is homotopy equivalent to $\check{C}\left(\mathcal{U}, \Omega_{D R}(U)\right)$.

Proof. - This statement follows from the homotopy equivalence of $\check{C}\left(\mathcal{U}, \Omega_{D R}(U)\right)$ and $\left|\Omega_{D R}\left(\mathcal{N} \mathcal{U}_{*}\right)\right|$ on one hand, and $\check{C}\left(\mathcal{U}, \Omega_{D R}(U)\right)$ and $\Omega_{D R}(X)$ on the other.

Let us give a more detailed description of this homotopy equivalence. Consider the map $w: \check{C}\left(\mathcal{U}, \Omega_{D R}(U)\right) \rightarrow\left|\Omega_{D R}\left(\mathcal{N U}_{*}\right)\right|$ : first we define it on the Čech cochains of degree 0 (we omit the $\otimes$ sign between elements of $\Omega_{D R}\left(\mathcal{N U}_{*}\right)$ and $\Omega_{*}(\triangle)$; we also omit $\left.1 \in \Omega_{*}(\triangle)\right)$ :

$$
w\left(\left\{h_{\alpha}\right\}_{\alpha}\right)=\left\{\bigoplus_{i} h_{i}, \bigoplus_{i \leqslant j}\left(\left(h_{i}\right)_{\left.\right|_{U_{i j}}} t_{0}+\left(h_{j}\right)_{\left.\right|_{U_{i j}}} t_{1}\right), \ldots\right\}
$$

so that on the $n$-th simplicial level we have

$$
w\left(\left\{h_{\alpha}\right\}_{\alpha}\right)_{n}=\bigoplus_{i_{0} \leqslant \ldots \leqslant i_{n}}\left(\left(h_{i_{0}}\right)_{\left.\right|_{U_{0} \ldots i_{n}}} t_{0}+\ldots+\left(h_{i_{n}}\right)_{U_{i_{0} \ldots i_{n}}} t_{n}\right) .
$$

Let us show that this element belongs to $\left|\Omega_{D R}\left(\mathcal{N} \mathcal{U}_{*}\right)\right|$. As before, it is enough to check only the relations, involving the (co)face and (co)degeneracy operations. So if we apply the coface operation in $\Omega_{D R}\left(\mathcal{N U}_{*}\right)$ direction, $\delta_{p} \otimes 1$, 
G. Sharygin

$p=0, \ldots, n+1$, to $w\left(\left\{h_{\alpha}\right\}_{\alpha}\right)_{n}$, we obtain

$$
\begin{aligned}
i_{p}^{*} \otimes 1 & \bigoplus \bigoplus_{i_{0} \leqslant \ldots \leqslant i_{n}}\left\{\left(h_{i_{0}}\right)_{\left.\right|_{U_{i_{0}} \ldots i_{n}}} t_{0}+\ldots+\left(h_{i_{n}}\right)_{\left.\right|_{U_{i_{0} \ldots i_{n}}}} t_{n}\right\} \\
= & \bigoplus_{i_{0} \leqslant \ldots \leqslant i_{p-1} \leqslant j \leqslant i_{p} \ldots \leqslant i_{n}}\left\{\left(h_{i_{0}}\right)_{\left.\right|_{U_{i_{0} \ldots j \ldots i_{n}}}} t_{0}+\ldots+\left(h_{i_{n}}\right)_{\left.\right|_{U_{i_{0} \ldots j \ldots i_{n}}}} t_{n}\right\},
\end{aligned}
$$

where $j$ on the right hand side stands in the $p$-th place and the term with $h_{j}$ is missing. On the other hand, if we apply $1 \otimes \partial_{p}$ to

$$
\begin{aligned}
& w\left(\left\{h_{\alpha}\right\}_{\alpha}\right)_{n} \\
& =\bigoplus_{i_{0} \leqslant \ldots \leqslant i_{n+1}}\left\{\left(h_{i_{0}}\right)_{\left.\right|_{U_{i_{0} \ldots i_{n}}}} t_{0}+\ldots+\left(h_{i_{n}}\right)_{\left.\right|_{U_{i_{0} \ldots i_{n}}}} t_{n}\right\} \\
& =\bigoplus_{i_{0} \leqslant \ldots \leqslant i_{p} \leqslant \ldots \leqslant i_{n+1}}\left\{\left(h_{i_{0}}\right)_{\left.\right|_{U_{i_{0} \ldots i_{p} \ldots i_{n+1}}}} t_{0}+\ldots+\left(h_{i_{n+1}}\right)_{\left.\right|_{U_{i_{0} \ldots i_{p} \ldots i_{n+1}}}} t_{n+1}\right\},
\end{aligned}
$$

we shall obtain the same result, see the definition of $\partial_{p}\left(t_{j}\right)$. Similarly, for all $q=0, \ldots, n-1$

$$
\begin{aligned}
& j_{q}^{*}\left(\left\{\left(h_{i_{0}}\right)_{\left.\right|_{U_{0} \ldots i_{n}}} t_{0}+\ldots+\left(h_{i_{n}}\right)_{\left.\right|_{U_{i_{0} \ldots i_{n}}}} t_{n}\right\}\right) \\
& =\left\{\begin{array}{l}
\left.\left(h_{i_{0}}\right)_{\left.\right|_{U_{i_{0} \ldots i_{n}}}} t_{0}+\ldots+\left(h_{i_{q}}\right)_{\left.\right|_{U_{0} \ldots i_{n}}}\left(t_{q}+t_{q+1}\right)+\ldots+\left(h_{i_{n}}\right)_{\left.\right|_{U_{i_{0} \ldots i_{n}}}} t_{n}\right\}, \\
\text { if } i_{q}=i_{q+1}, \\
0, \text { otherwise. }
\end{array}\right.
\end{aligned}
$$

We see that $\sigma_{q} \otimes 1\left(w\left(\left\{h_{\alpha}\right\}_{\alpha}\right)_{n}\right)=1 \otimes s_{q}\left(w\left(\left\{h_{\alpha}\right\}_{\alpha}\right)_{n-1}\right)$. Further, for $x=\left\{h_{\alpha \beta}\right\}_{\alpha \prec \beta}$, we put

$$
w(x)=\left\{0, w(x)_{1}, w(x)_{2}, \ldots\right\},
$$

where

$$
w(x)_{1}=\bigoplus_{i \leqslant j}\left(t_{0} d\left(t_{1} h_{i j}-t_{1} d\left(t_{0} h_{i j}\right)\right)\right)=\bigoplus_{i \leqslant j}-d t_{0} h_{i j}
$$

$$
\begin{gathered}
w(x)_{2}=\bigoplus_{i \leqslant j \leqslant k}\left(t_{0} d\left(t_{1}\left(h_{i j}\right)_{\left.\right|_{U_{i j k}}}+t_{2}\left(h_{i k}\right)_{\left.\right|_{i j k}}\right)+t_{1} d\left(-t_{0}\left(h_{i j}\right)_{\left.\right|_{i j k}}+t_{2}\left(h_{j k}\right)_{\left.\right|_{U_{i j k}}}\right)\right. \\
\left.+t_{2} d\left(-t_{0}\left(h_{i k}\right)_{\left.\right|_{U_{i j k}}}-t_{1}\left(h_{j k}\right)_{\left.\right|_{U_{i j k}}}\right)\right)
\end{gathered}
$$

In general, in the $n$-th simplicial degree there will stand

$$
w(x)_{n}=\bigoplus_{i_{0} \leqslant \ldots \leqslant i_{n}}\left(\sum_{k=0}^{n} t_{k} d\left(\sum_{j=0}^{n} t_{j}\left(\tilde{h}_{i_{k} i_{j}}\right)_{\left.\right|_{U_{i} \ldots i_{n}}}\right)\right) .
$$


Here we put $\tilde{h}_{k l}=h_{k l}, k<l$ and $\tilde{h}_{k l}=-h_{k l}$ otherwise, in particular, $\tilde{h}_{i i}=$ 0. In general, for $x=\left\{h_{\alpha_{0} \ldots \alpha_{n}}\right\}_{\alpha_{0} \prec \ldots \prec \alpha_{n}} \in \check{C}\left(\mathcal{U}, \Omega_{D R}(U)\right)$, the element $w(x)$ will consist of the following elements $w(x)_{p}$ belonging to the $p$-th simplicial degree (we omit the restriction signs):

$w(x)_{p}=\left\{\begin{array}{l}0, \quad \text { if } p<n, \\ \bigoplus_{i_{0} \leqslant \ldots \leqslant i_{p}}\left\{\sum_{k_{0}=0}^{p} t_{k_{0}} d\left\{\sum_{k_{1}=0}^{p} t_{k_{1}} \ldots d\left\{\sum_{k_{n}=0}^{p} t_{k_{n}}(-1)^{\sigma\left(k_{0}, \ldots, k_{n}\right)} h_{k_{0} \ldots k_{n}}\right\} \ldots\right\}\right\}, \\ \text { if } p \geqslant n .\end{array}\right.$

It is possible to check straightforwardly that these formulas give well-defined elements in $\left|\Omega_{D R}\left(\mathcal{N U}_{*}\right)\right|$ and that the map commutes with differentials. This way of proving is related with harsh computational difficulties. However

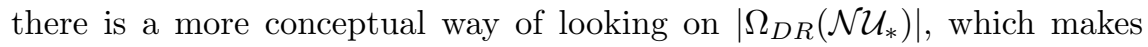
the map $w$ a particular case of the maps, we used earlier. The idea is very simple: let us look at $\left|\mathcal{N U}_{*}\right|$ as a topological space, glued of the cylinders $U_{\alpha \beta} \times \triangle^{1}, U_{\alpha \beta \gamma} \times \triangle^{2}, \ldots$; here we consider only nondegenerate simplices $(\alpha \beta \gamma)$ in the combinatoric nerve of $\mathcal{U}$. These subspaces are not open, but we can use them to define an open cover of $\left|\mathcal{N} \mathcal{U}_{*}\right|$ : put

$$
\widetilde{U_{\alpha}}=\bigcup_{\alpha \in \sigma} U_{\sigma} \times \triangle_{\alpha}^{|\sigma|} .
$$

The union is taken over all the simplices containing $\alpha$ in combinatorical nerve of $\mathcal{U}$, and $\triangle_{\alpha}^{|\sigma|},|\sigma|=\operatorname{dim} \sigma$, denotes the union of all the open hyperfaces of $\triangle^{|\sigma|}$, containing $\alpha$ as one of their vertices; i.e. $\triangle_{\alpha}^{|\sigma|}=\triangle^{|\sigma|}-\partial_{i} \triangle^{|\sigma|}$, where $\sigma=\left(\alpha_{0} \prec \ldots \prec \alpha_{i-1} \prec \alpha \prec \alpha_{i+1} \prec \ldots \prec \alpha_{|\sigma|}\right)$.

More generally, one can extend this definition to arbitrary simplex $\tau=$ $\left(\beta_{0}, \ldots, \beta_{k}\right)$ :

$$
\widetilde{U_{\tau}}=\bigcup_{\tau \subseteq \sigma} U_{\sigma} \times \triangle_{\tau}^{|\sigma|},
$$

where $\triangle_{\tau}^{|\sigma|}$ denotes the union of all faces of $\triangle^{|\sigma|}$, containing $\tau$ as their subface. One can embed $U_{\tau}$ into $\widetilde{U_{\tau}}$ regarding it as the "bottom" of all the cylinders in the union, i.e. putting $t_{i}=0$ for all $t_{i}$, which correspond to the vertices not in $\tau$. The following equality holds:

$$
\widetilde{U_{\tau_{1}}} \bigcap \widetilde{U_{\tau_{2}}}=\widetilde{U_{\tau_{1} \cup \tau_{2}}} \text {. }
$$

Sending $U_{\tau}$ to $\widetilde{U_{\tau}}$, gives a morphism $\mathcal{N}^{c o m b} \mathcal{U}_{*} \rightarrow \mathcal{N} \widetilde{\mathcal{U}}\left(\left|\mathcal{N} \mathcal{U}_{*}\right|\right)_{*}$, where we use the open cover $\widetilde{\mathcal{U}}=\left\{\widetilde{U_{\alpha}}\right\}$ to define the topological nerve of $\left|\mathcal{N U}_{*}\right| ; \mathcal{N}^{\text {comb }}$ 
denotes the combinatoric nerve of $\mathcal{U}$. Taking de Rham forms on both sides of this construction, we obtain a homomorphism of chain complexes (and even of differential graded algebras): $\tilde{i}: \check{C}\left(\mathcal{U}, \Omega_{D R}(U)\right) \rightarrow \check{C}\left(\tilde{\mathcal{U}}, \Omega_{D R}(\widetilde{U})\right)$, which sends the differential form $x$ on $U_{\tau}$ to the differential form $\tilde{x}$ on $\widetilde{U_{\tau}}$, equal to $x \otimes 1$.

Proposition 2.11. - The map $\tilde{i}$ is a quasi-isomorphism.

Proof. - It is enough to observe that $\tilde{i}$ composed with $i^{\prime}: \Omega_{D R}(X) \rightarrow$ $\check{C}\left(\mathcal{U}, \Omega_{D R}(U)\right)$, is equal to $i^{\prime} \circ Q_{\mathcal{N}}^{*}$. Since all the other maps here are quasiequivalences, so is $\tilde{i}$.

Now one can construct the map $w$ as the composition of $\tilde{i}$ and the inverse $\tilde{p}$ of $i^{\prime}$. In particular, in this way one obtains the formulas (2.24) and we can conclude that they are well-defined and commute with differentials.

Remark 2.12. - One can give an explicit formula for the homotopy inverse map of $w$. To this end consider the definite integral $\int_{\triangle^{n}}: \Omega_{n} \rightarrow \mathbb{R}$. It is easy to check, using the Stokes formula

$$
\int_{\triangle^{n}} d \omega=\int_{\partial \triangle^{n}} \omega
$$

that the map

$$
v=\prod_{n} n !\left(I d \otimes \int_{\triangle^{n}}\right):\left|\Omega_{D R}\left(\mathcal{N} \mathcal{U}_{*}\right)\right| \rightarrow \check{C}\left(\mathcal{U}, \Omega_{D R}(U)\right)
$$

commutes with the differentials. On the other hand, its composition with $w$ is equal to the identity. In fact $\int_{\triangle^{n}} t_{0} d t_{1} \ldots d t_{n}=\frac{1}{n !}$, while $\operatorname{deg}_{t} w(x)_{n} \leqslant$ $\operatorname{deg} x$ and the degrees are equal, only when $\operatorname{deg} x=n$, so $v$ is actually homotopy inverse to $w$.

\section{Homological monodromy and free loop spaces}

In this section we shall construct the homological monodromy map and discuss its properties. We begin with the holonomy map of a trivial bundle. We use the time-exponent description of holonomy and deduce the formula for its homological analogue. Then we discuss the analogous maps on the level of the free loop space and the gauge bundle of an arbitrary bundle and give a detailed description of these maps for the frame bundle of a vector bundle. In particular, we construct the bi-twisted tensor product, which models the gauge bundle. Finally, we formulate the comparison theorem for the homological and geometric maps. 


\subsection{Time ordered exponent and holonomy}

Let $P \rightarrow X$ be a principal $G$-bundle, and $\gamma:[0 ; 1] \rightarrow X$ a path in $X$. One can define the parallel transition (holonomy) of $P$ along $\gamma$, associated to a connection $A$ as follows. It is enough to define it for the associated vector bundle $E^{\rho}$ of $P$, where $\rho$ is a faithful representation of $G$. A local section of $E^{\rho}$ is covariantly constant along $\gamma$ with respect to the connection $A$, if the following linear differential equation holds:

$$
\nabla_{\frac{d}{d t}}(s)=\frac{d}{d t} s+\sum_{k} A_{k}\left(\frac{d}{d t}\right) \rho\left(h_{k}\right)(s)=0 .
$$

Here we regard $s$ as a $\mathbb{C}^{n}$-valued function on $[0 ; 1]\left(s(t) \in E_{\gamma(t)}^{\rho}\right.$ and vector bundles over a segment are trivial). For a vector $v \in E_{\gamma(0)}^{\rho}$ one can consider the unique solution $s_{v}$ of (3.1) with initial value $s_{v}(0)=v$. Then the holonomy is defined as the map, sending $v$ to $s_{v}(1)$. If we choose bases in the fibres of $E$ at $\gamma(0)$ and $\gamma(1)$, we shall obtain a matrix $M_{A} \in \rho(G)$. The map $\gamma \mapsto M_{A}(\gamma)$ satisfies the relation $M_{A}\left(\gamma_{1} * \gamma_{2}\right)=M_{A}\left(\gamma_{1}\right) M_{A}\left(\gamma_{2}\right)$ when the paths $\gamma_{i}$ are composable, and hence it defines a "group homomorphism" from the pointed loop space $\Omega X$ to $\rho(G)$ (recall, that $\Omega X$ is not a group or even a monoid itself, but only homotopy equivalent to a group).

From now on and through the end of this section, we will suppose that the principal bundle $P$ is trivial. Choose a global section of $P$ and pull the connection $A$ to a $\mathfrak{g}$-valued differential 1-form on $X$. It is well known, that one can write down the holonomy matrix $M_{A}$ in the terms of the so-called time-ordered exponent as follows:

$$
M_{A}(\gamma)=P \exp \int_{\gamma} A^{\rho} d t
$$

The right hand side of (3.2) is equal to the infinite sum of Chen's iterated integrals:

$$
P \exp \int_{\gamma} A^{\rho} d t=\sum_{n=0}^{\infty} \int_{\Delta^{n}} A^{\rho}\left(t_{1}\right) \ldots A^{\rho}\left(t_{n}\right) d t_{1} \ldots d t_{n},
$$

where $\Delta^{n}=\left\{\left(t_{1}, \ldots, t_{n}\right) \in \mathbb{R}^{n} \mid 0 \leqslant t_{1} \leqslant \ldots \leqslant t_{n} \leqslant 1\right\}$ is $n$-dimensional simplex. Consider the map $\Psi_{\gamma}: \Delta^{n} \rightarrow X ;\left(t_{1}, \ldots, t_{n}\right) \mapsto \gamma\left(\frac{1}{n}\left(t_{1}+\ldots+t_{n}\right)\right)$. It defines the inverse image map $\Psi_{\gamma}^{*}: \Omega_{D R}^{*}(X) \rightarrow \Omega_{D R}^{*}\left(\Delta^{n}\right)$. For $\omega \in$ $\Omega_{D R}^{1}(X)$, we define $\omega\left(t_{i}\right) \in C^{\infty}\left(\Delta^{n}\right)$ as the coefficients in the formula $\Psi_{\gamma}^{*}(\omega)=\sum_{i=1}^{n} \omega\left(t_{i}\right) d t_{i}$. One defines iterated integral $\int_{\Delta^{n}} A^{\rho}\left(t_{1}\right) \ldots A^{\rho}\left(t_{n}\right) d t_{1}$ $\ldots d t_{n}$ as

$$
\begin{aligned}
\int_{\Delta^{n}} A^{\rho}\left(t_{1}\right) \ldots A^{\rho}\left(t_{n}\right) d t_{1} \ldots d t_{n}= & \int_{0}^{1} A^{\rho}\left(t_{1}\right)\left[\int_{t_{1}}^{1} A^{\rho}\left(t_{2}\right)\left[\int_{t_{2}}^{1} \ldots\right] d t_{2}\right] d t_{1}(3.4 \\
& -333-
\end{aligned}
$$


The holonomy map defines the inverse image homomorphism $M_{A}^{*}: \Omega_{D R}(G)$ $\rightarrow \Omega_{D R}(\Omega X)$, where $\Omega X$ is the based loop space, see [8] for the definitions of its differential forms. Let us describe the $M_{A}^{*}$. In virtue of (1.7), it is enough to find $M_{A}^{*}\left(u_{i j}^{\rho}\right)$ for all representations $\rho$. Since $\Psi_{\gamma}^{*}\left(A^{\rho}\right)=\sum_{k} \Psi_{\gamma}^{*}\left(A_{k}\right) \rho\left(h_{k}\right)$, we see that $A^{\rho}\left(t_{i}\right)=\sum_{k} A_{k}\left(t_{i}\right) \rho\left(h_{k}\right)$, so

$$
M_{A}(\gamma)=\sum_{n,\left(k_{1}, \ldots, k_{n}\right)}\left(\int_{\Delta^{n}} A_{k_{1}}\left(t_{1}\right) \ldots A_{k_{n}}\left(t_{n}\right) d t_{1} \ldots d t_{n}\right) \rho\left(h_{k_{1}} \ldots h_{k_{n}}\right) .
$$

Here the summation is taken over all collections $\left(k_{1}, \ldots, k_{n}\right), k_{i}=1, \ldots, N$, and we denote the induced representation of the universal enveloping algebra of $\mathfrak{g}$ by the same symbol $\rho$. Clearly, the $(i, j)^{\text {th }}$ entry of this matrix is equal to

$$
M_{A}(\gamma)_{i j}=\sum_{n,\left(k_{1}, \ldots, k_{n}\right)}\left(\int_{\Delta^{n}} A_{k_{1}}\left(t_{1}\right) \ldots A_{k_{n}}\left(t_{n}\right) d t_{1} \ldots d t_{n}\right) \rho\left(h_{k_{1}} \ldots h_{k_{n}}\right)_{i j} .
$$

Now recall that the representation of Lie algebra is defined by the formula

$$
\rho(X)=\left.\frac{d}{d t}\right|_{t=0} \rho(\exp (t X)),
$$

for $X \in \mathfrak{g}$. Hence the pullback by $M_{A}$ of an arbitrary function $f \in \mathcal{A}(G)$ is

$$
M_{A}^{*}(f)=\left.\sum_{n,\left(k_{1}, \ldots, k_{n}\right)} \frac{1}{n !}\left(\int_{\Delta^{n}} A_{k_{1}}\left(t_{1}\right) \ldots A_{k_{n}}\left(t_{n}\right) d t_{1} \ldots d t_{n}\right) X_{k_{1}} \ldots X_{k_{n}}(f)\right|_{e}
$$

where $e$ denotes the unit of $G$, and we represent the basis $\left\{h_{i}\right\}_{i=1}^{N}$ by vector fields $\left\{X_{i}\right\}$ on $G$.

To go on with investigation of the holonomy map, we will need models for the De Rham algebra of loop spaces. One of the most convenient of them was suggested by Chen, see [8]. It is based on the notion of differentiable space and smooth plots. It is shown in [8] that for any 1-connected manifold $X$ there is a well-defined quasi-isomorphism $\sigma$, called the "iterated integral map", $\sigma: \bar{B}\left(\Omega_{D R}^{*}(X)\right) \rightarrow \Omega^{*}(\Omega X)$ (the ground field is $\Omega_{D R}(X)$-module via the restriction to the base-point $\left.x_{0}\right)$. In brief, one can define $\sigma$ as the composite $\operatorname{map} p_{*} \Phi_{n}^{*}$ in the diagramm:

$$
\begin{aligned}
& \Omega_{D R}\left(\Omega X \times \Delta^{n}\right) \stackrel{\Phi_{n}^{*}}{\longleftarrow} \Omega_{D R}\left(X^{\times n}\right) \stackrel{\supset}{\longleftarrow} \Omega_{D R}(X)^{\otimes n} \\
& \quad p_{*} \downarrow \\
& \Omega_{D R}(\Omega X),
\end{aligned}
$$


Here $\Phi_{n}\left(\gamma, t_{1}, \ldots, t_{n}\right)=\left(\gamma\left(t_{1}\right), \ldots, \gamma\left(t_{1}\right)\right), p: \Omega X \times \Delta^{n} \rightarrow \Omega X$ is the projection, and $p_{*}$ denotes the direct image, i.e. integration along the fibres of the projection. This is abbreviated to

$$
\sigma\left(\left[\omega_{1}|\ldots| \omega_{n}\right]\right)=\int_{\Delta^{n}} \omega_{1}\left(t_{1}\right) \ldots \omega_{n}\left(t_{n}\right) d t_{1} \ldots d t_{n} .
$$

In papers $[11,8]$ it is shown that the kernel of the Chen's iterated integral map $\sigma$ contains the subcomplex $\operatorname{ker}^{\prime} \sigma$ of $\bar{B}\left(\Omega_{D R}(X)\right)$, generated by the elements

$$
\left\{\left[w_{1}|\ldots| w_{n}\right] \in \bar{B}\left(\Omega_{D R}(X)\right) \mid \exists i=1, \ldots, n, w_{i} \in \Omega_{D R}^{0}(X)=C^{\infty}(X)\right\} .
$$

Under the assumptions we use in this paper, $\operatorname{ker}^{\prime} \sigma$ is acyclic, see [11] for details. We put

$$
N\left(\Omega_{D R}(X)\right) \stackrel{\text { def }}{=} \bar{B}\left(\Omega_{D R}(X)\right) / \operatorname{ker}^{\prime} \sigma .
$$

The factor-complex $N\left(\Omega_{D R}(X)\right)$ is a Hopf algebra. It is called the normalized bar-resolution, and $\operatorname{ker}^{\prime} \sigma$ is the normalization kernel. In what follows we shall usually deal with the normalized bar-resolution of $\Omega_{D R}(X)$. However we shall not make any notational difference between it and the usual bar-resolution, unless it is necessary.

\subsection{Homological Monodromy Map}

Our goal is to give an algebraic description of the inverse image map $M_{A}^{*}$, induced by the holonomy of a connection. First of all, using the Chen's iterated integral map $\sigma$ we can regard $M_{A}^{*}$ as a map from $\Omega_{\text {poly }}^{0}(G)=$ $\mathcal{A}(G)$ to the bar-resolution of $\Omega_{D R}(X)$. We assume the bundle to be trivial (e.g. take the bundle $\pi^{*}(P) \rightarrow P$, where $P \underset{G}{\stackrel{\pi}{\longrightarrow}} X$ is an arbitrary principal bundle). Then we define the homological monodromy map by the formula

$$
f \mapsto \widetilde{M}_{A}(f)=\sum_{n,(\bar{k})}\left(\left.X_{k_{1}} \ldots X_{k_{n}}(f)\right|_{e}\right)\left[A_{k_{1}}|\ldots| A_{k_{n}}\right],
$$

where $f \in \mathcal{A}(G)$ is a function and $\left\{X_{1}, \ldots, X_{N}\right\}$ is the basis of left-invariant vector fields on $G$. Recall that coproduct and shuffle products induce the commutative DG bialgebra structure on bar-complex of a commutative DG algebra.

Proposition 3.1. - The map $\widetilde{M}_{A}$ is a homomorphism of bialgebras.

Proof. - Consider the connection $A$ as a $\Omega_{D R}(X)$-valued differentiation on $\Omega_{\text {poly }}(G)$, or equivalently as a vector field on $G$ with coefficients 
in $\Omega_{D R}(X)$ :

$$
F_{A}=\sum_{i=1}^{N} A_{i} \otimes X_{i}
$$

We can map $\Omega_{D R}(X)$ to its bar-resolution, identifying it with the $\left(s \Omega_{D R}(X)\right)$ (shifting the dimension); then, in order to distinguish between the map to $\Omega_{D R}(X)$ and the corresponding map to the bar resolution, we shall write $\widehat{F}_{A}$ in the latter case; then $\widehat{F}_{A}$ is a differentiation on $\Omega_{\text {poly }}^{0}(G)$ with values in the bar-resolution. Formula (3.13) can be rewritten as

$$
\widetilde{M}_{A}(f)=\left.\sum_{n} \widehat{F}_{A}^{\otimes n}(f)\right|_{e}
$$

Here, $\widehat{F}_{A}^{\otimes n}$ is a $\bar{B}\left(\Omega_{D R}(X)\right) \otimes \mathcal{A}(G)$-valued differential operator on $\mathcal{A}(G)$ :

$$
\widehat{F}_{A}^{\otimes n}(f)=\sum_{(\bar{k})}\left[A_{k_{1}}|\ldots| A_{k_{n}}\right] \otimes X_{k_{1}} \ldots X_{k_{n}}(f) .
$$

In effect to any DG algebra $\Omega$ and any linear map $\alpha: \mathfrak{g} \rightarrow \Omega$, one can associate an $\Omega$-valued vector field on $G$

$$
F_{\alpha} \stackrel{\text { def }}{=} \sum_{i=1}^{N} \alpha\left(X_{i}\right) \otimes X_{i} .
$$

The corresponding maps to the bar-resolution of $\Omega$ we shall denote by $\widehat{F}_{\alpha}$. Observe, that one can rewrite the equations (3.15), (3.16) in the terms of shuffle products as

$$
\widetilde{M}_{A}(f)=\left.\sum_{n} \frac{1}{n !} \widehat{F}_{A}^{\operatorname{sh}(n)}(f)\right|_{e}
$$

where

$$
\begin{aligned}
\operatorname{sh}\left(\widehat{F}_{\alpha_{1}}, \ldots, \widehat{F}_{\alpha_{n}}\right)(f) \stackrel{\text { def }}{=} & \sum_{\sigma \in \Sigma_{n},(\bar{k})}(-1)^{\tilde{\sigma}}\left[\alpha_{\sigma^{-1}(1)}\left(h_{k_{1}}\right)|\ldots| \alpha_{\sigma^{-1}(n)}\left(h_{k_{n}}\right)\right] \\
& \otimes X_{k_{1}} \ldots X_{k_{n}} .
\end{aligned}
$$

Now the equation $\widetilde{M}_{A}(f g)=\operatorname{sh}\left(\widetilde{M}_{A}(f), \widetilde{M}_{A}(g)\right)$ follows from the Leibnitz rule for $\widehat{F}_{A}$ (with respect to the shuffle product). $\left.\widehat{F}_{A}\right|_{e}$ also verifies the Leibnitz rule with respect to the coproduct:

$$
\begin{gathered}
\left(\left.\widehat{F}_{A}\right|_{e} \otimes \epsilon+\left.\epsilon \otimes \widehat{F}_{A}\right|_{e}\right)\left(\Delta_{\mathcal{A}(G)}(f)\right)=\Delta_{\bar{B}\left(\Omega_{D R}(X)\right)}\left(\left.\widehat{F}_{A}\right|_{e}\right) . \\
-336-
\end{gathered}
$$


We compute:

$$
\begin{aligned}
\left(\widehat{F}_{A} \otimes \epsilon+\epsilon \otimes \widehat{F}_{A}\right)\left(\Delta_{\mathcal{A}(G)}(f)\right) & =\left(\widehat{F}_{A} \otimes \epsilon+\epsilon \otimes \widehat{F}_{A}\right)\left(\sum_{(f)} f_{(1)} \otimes f_{(2)}\right) \\
& =\sum_{(f)}\left\{\widehat{F}_{A}\left(f_{(1)}\right) \otimes \epsilon\left(f_{(2)}\right)+\epsilon\left(f_{(1)}\right) \otimes \widehat{F}_{A}\left(f_{(2)}\right)\right\} \\
& =\widehat{F}_{A}(f) \otimes 1+1 \otimes \widehat{F}_{A}(f) \\
& =\Delta_{\bar{B}\left(\Omega_{D R}(X)\right)}\left(\widehat{F}_{A}(f)\right) .
\end{aligned}
$$

As above, we conclude that $\widetilde{M}_{A}$ is a homomorphism of coalgebras too.

Now, since $\widetilde{M}_{A}$ is a homomorphism of commutative bialgebras, we can extend it to the algebra $\Omega_{\text {poly }}(G)$, so that the extension will also be a homomorphism of graded bialgebras. Our next purpose is to describe this homomorphism in higher dimensions.

Let $\alpha$ and $\beta$ be two homogeneous maps $\alpha, \beta: \mathfrak{g} \rightarrow \Omega$ of degrees $|\alpha|,|\beta|>$ 0 respectively ( $\Omega$ is a commutative DG algebra). Then in the notation, explained above, we have (see (3.19)):

$$
\begin{array}{r}
\left(d_{B} \otimes 1\right)\left(\operatorname{sh}\left(\widehat{F}_{\alpha}, \widehat{F}_{\beta}\right)(f)\right)=\left(\operatorname{sh}\left(\widehat{F}_{d \alpha}, \widehat{F}_{\beta}\right)\right. \\
+(-1)^{|\alpha|-1} \operatorname{sh}\left(\widehat{F}_{\alpha}, \widehat{F}_{d \beta}\right) \\
\left.-(-1)^{|\alpha|-1}\left[\widehat{F}_{\alpha}, F_{\beta}\right]\right)(f),
\end{array}
$$

for all $f \in \mathcal{A}(G)$. Here $\left[F_{\alpha}, F_{\beta}\right]=\sum_{i, k=1}^{N} \alpha\left(h_{i}\right) \beta\left(h_{k}\right) \otimes\left[X_{i}, X_{k}\right]$ is the commutator of vector fields $F_{\alpha}$ and $F_{\beta}$. In fact, first two terms on the right side of (3.21) are obvious. They correspond to the first part $\left(d_{0}\right)$ of the barresolution's differential. Let's show, that the rest of this differential gives the third term. We compute

$$
\begin{array}{r}
\left(d_{1} \otimes 1\right)\left(\operatorname{sh}\left(\widehat{F}_{\alpha}, \widehat{F}_{\beta}\right)(f)\right)=\left(d_{1} \otimes 1\right)\left(\sum_{i, k=1}^{N}\left[\alpha\left(h_{i}\right) \mid \beta\left(h_{k}\right)\right] \otimes X_{i} X_{k}(f)\right) \\
+(-1)^{(|\alpha|-1)(|\beta|-1)}\left(d_{1} \otimes 1\right)\left(\sum_{i, k=1}^{N}\left[\beta\left(h_{i}\right) \mid \alpha\left(h_{k}\right)\right] \otimes X_{i} X_{k}(f)\right) \\
=(-1)^{|\alpha|-1} \sum_{i, k=1}^{N} \alpha\left(h_{i}\right) \beta\left(h_{k}\right) \otimes\left(X_{i} X_{k}(f)-X_{k} X_{i}(f)\right)=\left[\widehat{F_{\alpha}, F_{\beta}}\right](f) .
\end{array}
$$

This formula easily generalizes to the $n$-fold shuffle product of such maps:

$$
\begin{aligned}
& \left(d_{\bar{B}\left(\Omega_{D R}(X)\right)} \otimes 1\right) \operatorname{sh}\left(\widehat{F}_{\alpha_{1}}, \ldots, \widehat{F}_{\alpha_{n}}\right)=\sum_{k=1}^{n}(-1)^{\varepsilon_{k}} \operatorname{sh}\left(\widehat{F}_{\alpha_{1}}, \ldots, \widehat{F}_{d \alpha_{k}}, \ldots, \widehat{F}_{\alpha_{n}}\right) \\
& +\sum_{i<j}(-1)^{\varepsilon_{i j}} \operatorname{sh}(\left[F_{\alpha_{i}}, F_{\alpha_{j}}\right], F_{\alpha_{1}}, \ldots, \underbrace{\widehat{\imath}}_{i}, \ldots, \underbrace{\widehat{\gamma}}_{j}, \ldots, F_{\alpha_{n}}) .
\end{aligned}
$$


These formulae should be slightly modified, when some of the maps take values in $\Omega^{0}$ : we shall assume that the algebra $\Omega$ is equipped with an augmentation $\Omega \rightarrow \mathbb{C}$. In the case of de Rham algebras of a manifold $X$, the augmentation is given by evaluating a function $f$ in a point $x_{0}$. Then, if $|\alpha|=0$ one should add the terms $\left.\alpha\right|_{x_{0}}\left(\widehat{F}_{\beta}(f)\right)-\widehat{F}_{\beta}\left(\left.\alpha\right|_{x_{0}}(f)\right)=\left[\left.\alpha\right|_{x_{0}}, \widehat{F}_{\beta}\right](f)$ to the right hand side of (3.21). Here we regard $\left.\alpha\right|_{x_{0}}$ as a $\mathbb{C} \subseteq \bar{B}\left(\Omega_{D R}(X)\right)$ valued vector field on $G$. Respectively, if $\left|\alpha_{1}\right|=0$, then one should add $\left[\left.\alpha_{1}\right|_{x_{0}}, \operatorname{sh}\left(\widehat{F}_{\alpha_{2}}, \ldots, \widehat{F}_{\alpha_{n}}\right)\right]$ to the formula (3.23).

The shuffle product of vector fields, defined by equation (3.19) is graded commutative: let us compute in the case of two vector fields for example:

$$
\begin{aligned}
\operatorname{sh}\left(\widehat{F}_{\alpha}, \widehat{F}_{\beta}\right)(f)= & \sum_{i, k=1}^{N}\left[\alpha\left(h_{i}\right) \mid \beta\left(h_{k}\right)\right] \otimes X_{i} X_{k}(f) \\
& +(-1)^{(|\alpha|-1)(|\beta|-1)} \sum_{i, k=1}^{N}\left[\beta\left(h_{i}\right) \mid \alpha\left(h_{k}\right)\right] \otimes X_{i} X_{k}(f) \\
= & (-1)^{(|\alpha|-1)(|\beta|-1)} \operatorname{sh}\left(\widehat{F}_{\beta}, \widehat{F}_{\alpha}\right)(f),
\end{aligned}
$$

for all $f \in \mathcal{A}(G)$. We shall use this observation below.

\subsection{Homological monodromy map and the gauge transformations}

Let the group $G$ act on itself by conjugations. Consider the induced action on the algebra of smooth functions $\mathcal{A}(G), f \mapsto f^{g}, f^{g}(x)=f\left(g^{-1} x g\right)$. The purpose of this section is to determine the equivariance properties of the map $\widetilde{M}_{A}$ with respect to this action.

To answer this question we first recall, that any map $g: X \rightarrow G$ induces an endomorphism of the trivial principal bundle $X \times G$; in fact, the endomorphisms of $X \times G$ can be identified with the set of all maps $X \rightarrow G$ with pointwise multiplication. In particular, if we work with the pullback bundle $\pi^{*}(P)=P \times G$, then one can regard the transformations of $P$ as those transformations of $\pi^{*}(P)$, which can be pulled down to the bundle $P$. This amounts to considering the subgroup of $A d_{G}$-equivariant maps $g: P \rightarrow G, g(p h)=h^{-1} g(p) h$. Thus the general case can be reduced to the trivial one, which we consider here. Then, any map $g$ induces gauge transformation of connection form on $X$, see (1.11). We claim that the homological monodromy map $\widetilde{M}_{A}$ intertwines the adjoint action of $G$ on itself with the action, induced on the image of $\widetilde{M}_{A}$ by the gauge transformations. Here we identify an element $g \in G$ with the gauge transformation, induced by the corresponding constant map. 
The statement will follow from the explicit description of the action on the level of normalized bar-resolution. Since we assumed that $G$ was connected, it is enough to consider the infinitesimal part of the action. So we identify $\mathfrak{g}$ with the space of left-invariant vector fields on $G$ and let it act on $\mathcal{A}(G)$ by Lie derivatives and ask about the way $\widetilde{M}_{A}$ commutes with this action. Similarly, we can consider only infinitesimal gauge transformations: let the $g=\exp (t a), t \rightarrow 0$, where $a: X \rightarrow \mathfrak{g}$. Then one can write down (1.11) as

$$
A \mapsto A+t \delta_{a}(A)+\ldots=A+t([A, a]+d a)+\ldots,
$$

here $+\ldots$ denotes the sum of the terms of degrees $\geqslant 2$ in $t$. If $a(x)=$ $\sum_{k=1}^{N} a_{k}(p) h_{k}, a_{i} \in C^{\infty}(X)$, then $\delta_{a}(A)_{k}=\sum_{i, j=1}^{N} C_{i j}^{k} A_{i} a_{j}+d a_{k}, C_{i j}^{k}$ are the structure constants of $\mathfrak{g}$. Under this transformation, the vector field $F_{A}$ transforms as

$$
F_{A+t \delta_{a}(A)+\ldots}=F_{A}+t\left(F_{[A, a]}+F_{d a}\right)+\ldots=F_{A}+t\left(\left[F_{A}, F_{a}\right]+F_{d a}\right)+\ldots
$$

Now, using the equation (3.13), we compute:

$$
\begin{aligned}
& \widetilde{M}_{A+t \delta_{a}(A)+\ldots}(f) \\
= & \left.\sum_{n=0}^{\infty} \frac{1}{n !}\left(\sum_{k=0}^{n}\left(\begin{array}{l}
n \\
k
\end{array}\right) t^{n-k} \operatorname{sh}(\underbrace{\widehat{F}_{A}, \ldots, \widehat{F}_{A}}_{k \text { times }}, \widehat{F}_{\delta_{a}(A)}, \ldots, \widehat{F}_{\delta_{a}(A)})\right)(f)\right|_{e}+\ldots \\
= & \widetilde{M}_{A}(f)+t \sum_{n=1}^{\infty} \frac{1}{(n-1) !} \operatorname{sh}\left(\widehat{F}_{\delta_{a}(A)}, \widehat{F}_{A}, \ldots, \widehat{F}_{A}\right)+\ldots
\end{aligned}
$$

where ... means quadratic and higher order terms. Here we used the graded commutativity of the shuffles of vector fields. Thus, the linear part of the gauge transformation of $\widetilde{M}_{A}$ is equal to

$$
\widetilde{M}_{A}^{a}=\left.\frac{d}{d t}\left(\widetilde{M}_{A^{g}}\right)\right|_{t=0}=\sum_{n=0}^{\infty} \frac{1}{n !} \operatorname{sh}(\widehat{F}_{\delta_{a}(A)}, \underbrace{\widehat{F}_{A}, \ldots, \widehat{F}_{A}}_{n}) .
$$

Let $\alpha_{1}, \ldots, \alpha_{n}: \mathfrak{g} \rightarrow \Omega_{D R}(X)$ be a collection of linear maps. For the sake of simplicity we shall assume, that they all are of odd degrees. Then using (3.23) we obtain:

$$
\begin{aligned}
& d_{\bar{B}\left(\Omega_{D R}(X)\right)}\left(\left.\operatorname{sh}\left(\widehat{F}_{a}, \widehat{F}_{\alpha_{1}}, \ldots, \widehat{F}_{\alpha_{n}}\right)(f)\right|_{e}\right) \\
& =\left.\operatorname{sh}\left(\widehat{F}_{d a}, \widehat{F}_{\alpha_{1}}, \ldots, \widehat{F}_{\alpha_{n}}\right)(f)\right|_{e}+\left.\sum_{i=1}^{n} \operatorname{sh}\left(\widehat{F}_{a}, \widehat{F}_{\alpha_{1}}, \ldots, \widehat{F}_{d \alpha_{i}}, \ldots, \widehat{F}_{\alpha_{n}}\right)(f)\right|_{e} \\
& -339-
\end{aligned}
$$




$$
\begin{aligned}
& +\left.\sum_{i=1}^{n} \operatorname{sh}(\widehat{F}_{\left[\alpha_{i}, a\right]}, \widehat{F}_{\alpha_{1}}, \ldots, \underbrace{\widehat{\gamma}}_{i}, \ldots, \widehat{F}_{\alpha_{n}})(f)\right|_{e} \\
& +\left.\sum_{1 \leqslant i<j \leqslant n} \operatorname{sh}\left(\widehat{F}_{a}, \widehat{F}_{\left[\alpha_{i}, \alpha_{j}\right]}, \widehat{F}_{\alpha_{1}}, \ldots, \widehat{F}_{\alpha_{n}}\right)(f)\right|_{e}+\left.\left[\left.a\right|_{x_{0}}, \operatorname{sh}\left(\widehat{F}_{\alpha_{1}}, \ldots, \widehat{F}_{\alpha_{n}}\right)\right](f)\right|_{e} .
\end{aligned}
$$

Taking into consideration, the definition of $\operatorname{ker}^{\prime} \sigma$, the kernel of the Chen's iterated integral map, we have the following relation

$$
\begin{aligned}
\left.\operatorname{sh}\left(\widehat{F}_{d a}, \widehat{F}_{\alpha_{1}}, \ldots, \widehat{F}_{\alpha_{n}}\right)(f)\right|_{e} & +\left.\sum_{i=1}^{n} \operatorname{sh}(\widehat{F}_{\left[\alpha_{i}, a\right]}, \widehat{F}_{\alpha_{1}}, \ldots, \underbrace{\widehat{\gamma}}_{i}, \ldots, \widehat{F}_{\alpha_{n}})(f)\right|_{e} \\
& \left.\equiv\left[\operatorname{sh}\left(\widehat{F}_{\alpha_{1}}, \ldots, \widehat{F}_{\alpha_{n}}\right),\left.a\right|_{x_{0}}\right](f)\right|_{e}\left(\bmod \left(\operatorname{ker}^{\prime} \sigma\right)\right)
\end{aligned}
$$

Let's apply this formula to $\widetilde{M}_{A}^{a}(f)$. We compute:

$$
\begin{aligned}
& \operatorname{sh}(\widehat{F}_{d a},\left.\underbrace{\widehat{F}_{A}, \ldots, \widehat{F}_{A}}_{n}(f)\right|_{e} \equiv-\left.n \operatorname{sh}(\widehat{F}_{[A, a]}, \underbrace{\widehat{F}_{A}, \ldots, \widehat{F}_{A}}_{n-1})(f)\right|_{e}+\left[\operatorname{sh}(\underbrace{\widehat{F}_{A}, \ldots, \widehat{F}_{A}}_{n}),\right. \\
& \left.\left.a\right|_{x_{0}}\right]\left.(f)\right|_{e} \text {, }
\end{aligned}
$$

(modulo $\operatorname{ker}^{\prime} \sigma$ ) and hence

$$
\begin{aligned}
& \left.\sum_{n} \frac{1}{n !} \operatorname{sh}(\widehat{F}_{d a}, \underbrace{\widehat{F}_{A}, \ldots, \widehat{F}_{A}}_{n})(f)\right|_{e} \equiv-\left.\sum_{n} \frac{1}{(n-1) !} \operatorname{sh}(\widehat{F}_{[A, a]}, \underbrace{\widehat{F}_{A}, \ldots, \widehat{F}_{A}}_{n-1})(f)\right|_{e} \\
& +\left.\left[\sum_{n} \frac{1}{n !} \operatorname{sh}(\underbrace{\widehat{F}_{A}, \ldots, \widehat{F}_{A}}_{n}),\left.a\right|_{x_{0}}\right](f)\right|_{e} .
\end{aligned}
$$

Finally, we see, that modulo $\operatorname{ker}^{\prime} \sigma \widetilde{M}_{A}^{a}(f) \equiv\left[\widetilde{M}_{A},\left.a\right|_{x_{0}}\right](f)$. Exponentiating this equality, we conclude, that the normalized image of $\widetilde{M}_{A}$ verifies the following equation (recall, that $g$ is a map $g: X \rightarrow G$ ):

$$
\widetilde{M}_{A^{g}}(f)=\widetilde{M}_{A}\left(f^{g^{-1}\left(x_{0}\right)}\right)
$$

where for any $k \in G, f \mapsto f^{k}$ is the action of $G$ on $\mathcal{A}$, induced by the conjugation: $f^{g}(x)=f\left(g^{-1} x g\right)$. This is also true for the induced action on $\Omega_{G}$. We see, that $\widetilde{M}_{A^{g}}(\omega)=\widetilde{M}_{A}(\omega)$, if $\omega$ is an $A d$-invariant differential form on $G$. For a constant map $g,(3.31)$ reduces just to

$$
\widetilde{M}_{A}\left(f^{g^{-1}}\right)=\widetilde{M}_{A^{g}}(f) .
$$




\subsection{Homological monodromy of gauge bundles}

Let $P$ be an arbitrary principal bundle. Consider the "gauge bundle" $P_{A d}$, associated with $P$. Recall that $P_{A d}=P \times_{A d} G$; let $\pi_{A d}$ denote the corresponding projection. Sections of $P_{A d}$ give automorphisms of $P$, in particular, there is the "unit" section of this bundle, corresponding to the identity. Consider the following DG algebra associated with $P_{A d}$ :

$$
\begin{aligned}
\Omega_{v p o l y}^{*}\left(P_{A d}\right) & =\Omega_{D R}(P) \otimes_{G} \Omega_{\text {poly }}^{*}(G) \\
& =\left\{\omega \otimes_{\varphi} \in \Omega_{D R}(P) \otimes \Omega_{\text {poly }}^{*}(G) \mid \omega^{g} \otimes \varphi=\omega \otimes \varphi^{g^{-1}}, g \in G\right\} .
\end{aligned}
$$

Here $G$ acts on $\Omega_{\text {poly }}^{*}(G)$ by conjugations. One should regard $\Omega_{v p o l y}^{*}\left(P_{A d}\right)$ as the algebra of differential forms on $P_{A d}$ which are polynomial in the direction of the fibre. There is a map $1^{*}: \Omega_{v p o l y}^{*}\left(P_{A d}\right) \rightarrow \Omega_{D R}(X)$, induced by the counit of $\Omega_{\text {poly }}^{*}(G)$, i.e. by the inclusion of unit in $G$. Let $A=\sum_{k} A_{k} \otimes$ $h_{k}$ be the global connection form on the trivial bundle $\pi^{*}(P)=P \times G$, induced from a connection on $P$. Identifying $h_{k}$ with the corresponding Lie derivatives in $\Omega_{\text {poly }}^{*}(G)$, we associate to $A$ a vector field on $G$ with values in $\Omega_{D R}(P)$. We can extend it trivially to $P \times G$ and then restrict to the $G$-invariant forms $\left(\Omega_{D R}(P) \otimes \Omega_{\text {poly }}^{*}(G)\right)^{G}=\Omega_{v p o l y}^{*}\left(P_{A d}\right)$. Moreover, the equivariance of $A$ show that the image of this map still belongs to $\Omega_{v p o l y}^{*}\left(P_{A d}\right)$. Iterating it and evaluating the result at the unit section, we obtain a map $\widetilde{T}_{A}: \Omega_{v p o l y}^{0}\left(P_{A d}\right) \rightarrow N H\left(\Omega_{D R}(X)\right)$

$\widetilde{T}_{A}(a \otimes b)=\sum_{n=0}^{\infty}(\underbrace{I d \otimes \ldots \otimes I d}_{n} \otimes 1^{*}) A^{\otimes n}(a \otimes b), a \in \Omega_{D R}^{0}(P), b \in \Omega_{\text {poly }}^{0}(G)$.

Here $N H\left(\Omega_{D R}(X)\right)$ denotes the normalized (or reduced) Hochschild complex of $\Omega_{D R}(X)$. It is defined in the same manner as $N\left(\Omega_{D R}(X)\right)$, i.e. all instances of degree 0 forms in the first $n$ places and their differentials are quotient out. This complex is a model for the algebra of differential de Rham forms on the free loop space of $X$, when $X$ is 1-connected, see for example $[11,8,9]$. The fact that the image of $\widetilde{T}_{A}$ is indeed in $N H\left(\Omega_{D R}(X)\right)$, follows from the next observations: one can identify $\Omega_{D R}(X)$ with the subalgebra of basic forms in $\Omega_{D R}(P)$. Then the invariance of $A$

$$
A^{g}(b)=A\left(b^{g^{-1}}\right)=A(b) \text { for all } g \in G
$$

(it follows from the definitions of $1^{*}$ and the adjoint action) shows that the image of $\widetilde{T}_{A}$ consists of $G$-invariant elements. And the second condition of the basic elements follows from the fact that we consider reduced Hochschild complex $N H\left(\Omega_{D R}(X)\right)$. A reasoning, similar to the one we used 
in section 3.2, starting with an explicit formula of the time-ordered exponent, shows that the map $\widetilde{T}_{A}$ is in fact a homological counterpart of the holonomy map $T_{A}$, that associates an automorphism of $P$ to a section of the infinite-dimensional bundle $\mathcal{L} X \rightarrow X$. One can now repeat all the reasoning from previous sections to show that $\widetilde{T}_{A}$ can be extended to a homomorphism of differential graded algebras and that it intertwines coproduct structures on both sides: on the left it is induced from the product $P_{A d} \times_{X} P_{A d} \rightarrow P_{A d}$ and on the right it is a generalization of the bar-construction coproduct. In particular one can show, that if $g: X \rightarrow G$ is a gauge transformation, then

$$
T_{A^{g}}(\alpha)=T_{A}\left(\alpha^{g^{-1}}\right) \text {, for any } \alpha \in \Omega_{v p o l y}^{*}\left(P_{A d}\right) \text {. }
$$

Here the action of $g$ on the algebra $\Omega_{v p o l y}^{*}\left(P_{A d}\right)$ is induced from its action on functions, which is given by the product of the arguments by $g$ from the left (recall, that the bundle $P_{A d}$ is in fact a group fibration, in particular, one can multiply its elements by its sections on both sides).

This construction is quite convenient, but not very explicit, since it is not easy to see why the image of $\widetilde{T}_{A}$ belongs to $N H\left(\Omega_{D R}(X)\right)$. One can amend it using the Cech complex of the trivializing open cover $\mathcal{U}$ of $P$ and obtain the monodromy map with values in the normalized Hochschild complex of Cech complex $\check{C}\left(\mathcal{U}, \Omega_{D R}(U)\right)$ like we did it in previous sections. We shall not prove this in full generality here, below we shall discuss the case of frame bundle of a vector bundle in full detail.

\subsection{The gauge bundle and twisting cochains}

Now we are going to give a purely algebraic description of the homological monodromy map in case of free loop space, involving twisting cochain. It should be a map from (a model of) the algebra of differential forms on the gauge bundle to the normalized Hochschild complex of a differential graded "model" of the base. The main difficulty that one meets here is that not every model of the gauge bundle is suitable for this. So we introduce complex $K \hat{\otimes}_{\phi} \Omega$, and prove that it can be regarded as a model for the gauge bundle.

Let $K$ (resp. $\Omega$ ) be a DG coalgebra (resp. algebra); let $K$ be coaugmented and $\phi: K^{*} \rightarrow \Omega^{*+1}$ be a twisting cochain. Instead of the usual twisted tensor product $K \otimes_{\phi} \Omega$ one can consider the following generalization thereof, which one can call bitwisted:

DEFinition 3.2. - The tensor product $K \hat{\otimes}_{\phi} \Omega$ is as before linearly isomorphic to the graded tensor product of $K$ and $\Omega$ and the differential is 
given by the formula:

$$
\begin{aligned}
\hat{d}_{\phi}(\alpha \otimes \beta)=d(\alpha) & \otimes \beta+(-1)^{|\alpha|} \alpha \otimes d \beta+\alpha^{(1)} \\
& \otimes \phi\left(\alpha^{(2)}\right) \beta+(-1)^{\left(\left|\alpha^{(1)}\right|+1\right)\left(\left|\alpha^{(2)}\right|+|\beta|\right)} \alpha^{(2)} \otimes \beta \phi\left(\alpha^{(1)}\right) .
\end{aligned}
$$

An easy calculation shows that $\hat{d}_{\phi}^{2}=0$. The following statement is the main theorem of this paragraph. It is a direct analogy of the Brown's theorem, see [7]. We give a brief proof for the sake of the self-containedness of our paper:

Proposition 3.3. - Let $\Omega$ be unital and $K$ be connected (i.e. $K^{0}$ is isomorphic to the ground field) and let $\phi$ be a twisted cochain associated with the principal bundle $P$. Then the cohomology of the $K \hat{\otimes}_{\phi} \Omega$ is isomorphic to the cohomology of the gauge bundle $P \times_{A d} G$.

Proof. - First of all, consider the following intermediate complex:

$$
C(K, \Omega ; \phi)=\bigoplus_{p, q=0}^{\infty}\left(K \otimes \bar{K}^{\otimes^{p}} \otimes K\right) \otimes\left(\Omega \otimes \bar{\Omega}^{q} \otimes \Omega\right),
$$

where $\bar{K}=K / 1$ ( 1 is the image of the coaugmentation of $K$ ) $\bar{\Omega}=\Omega / 1$, the grading is given by

$$
\begin{gathered}
\operatorname{deg} a\left[k_{1}|\ldots| k_{p}\right] b \otimes \alpha\left[\omega_{1}|\ldots| \omega_{q}\right] \beta=\operatorname{deg} a+\operatorname{deg} b+\sum \operatorname{deg} k_{i}+p+\operatorname{deg} \alpha \\
+\sum \operatorname{deg} \omega_{j}-q+\operatorname{deg} \beta,
\end{gathered}
$$

we use $\mid$ instead of the tensor signs. Since we have assumed that $\Omega$ is connected, there's no problem with negative degrees. The differential in this complex shall be given by the formula

$$
\begin{gathered}
d\left(a\left[k_{1}|\ldots| k_{p}\right] b \otimes \alpha\left[\omega_{1}|\ldots| \omega_{q}\right] \beta\right)=d a\left[k_{1}|\ldots| k_{p}\right] b \otimes \alpha\left[\omega_{1}|\ldots| \omega_{q}\right] \beta \\
+\sum_{i=1}^{p}(-1)^{\epsilon_{i}} a\left[k_{1}|\ldots| d k_{i}|\ldots| k_{p}\right] \otimes \alpha\left[\omega_{1}|\ldots| \omega_{q}\right] \beta \\
+(-1)^{\epsilon_{p}} a\left[k_{1}|\ldots| k_{p}\right] d b \otimes \alpha\left[\omega_{1}|\ldots| \omega_{q}\right] \beta \\
+(-1)^{\epsilon}\left(a\left[k_{1}|\ldots| k_{p}\right] b \otimes d \alpha\left[\omega_{1}|\ldots| \omega_{q}\right] \beta\right. \\
+\sum_{j=1}^{q}(-1)^{\eta_{j}} a\left[k_{1}|\ldots| k_{p}\right] \otimes \alpha\left[\omega_{1}|\ldots| d \omega_{j} \mid \omega_{q}\right] \beta \\
\left.+(-1)^{\eta_{q}} a\left[k_{1}|\ldots| k_{p}\right] \otimes \alpha\left[\omega_{1}|\ldots| \omega_{q}\right] d \beta\right) \\
-343-
\end{gathered}
$$




\section{G. Sharygin}

$$
\begin{aligned}
& +a^{(1)}\left[a^{(2)}\left|k_{1}\right| \ldots \mid k_{p}\right] b \otimes \alpha\left[\omega_{1}|\ldots| \omega_{q}\right] \beta \\
& +\sum_{i=1}^{p}(-1)^{\epsilon_{i}} a\left[k_{1}|\ldots| k_{i}^{(1)}\left|k_{i}^{(2)}\right| \ldots \mid k_{p}\right] b \otimes \alpha\left[\omega_{1}|\ldots| \omega_{q}\right] \beta \\
& +(-1)^{\epsilon_{p}} a\left[k_{1}|\ldots| k_{p} \mid b^{(1)}\right] b^{(2)} \otimes \alpha\left[\omega_{1}|\ldots| \omega_{q}\right] \beta \\
& +(-1)^{\epsilon}\left(a\left[k_{1}|\ldots| k_{p}\right] b \otimes \alpha \omega_{1}\left[\omega_{2}|\ldots| \omega_{q}\right] \beta\right. \\
& +\sum_{j=1}^{q-1}(-1)^{\eta_{j}} a\left[k_{1}|\ldots| k_{p}\right] b \otimes \alpha\left[\omega_{1}|\ldots| \omega_{j} \omega_{j+1} \mid \omega_{q}\right] \beta \\
& \left.+(-1)^{\eta_{q}} a\left[k_{1}|\ldots| k_{p}\right] b \otimes \alpha\left[\omega_{1}|\ldots| \omega_{q-1}\right] \omega_{q} \beta\right) \\
& +(-1)^{\chi_{1}} a^{(2)}\left[k_{1}|\ldots| k_{p}\right] b \otimes \alpha\left[\omega_{1} \ldots \mid \omega_{q}\right] \beta \phi\left(a^{(1)}\right) \\
& +(-1)^{\chi_{2}} a\left[k_{1}|\ldots| k_{p}\right] b^{(1)} \otimes \phi\left(b^{(2)}\right) \alpha\left[\omega_{1}|\ldots| \omega_{q}\right] \beta .
\end{aligned}
$$

Here $\epsilon_{i}=|a|+\sum_{l=1}^{i-1}\left|k_{l}\right|-i+1, \epsilon=|a|+\sum_{i=1}^{p}\left|k_{i}\right|-p+|b|, \eta_{j}=|\alpha|+$ $\sum_{m=1}^{j-1}\left|\omega_{m}\right|+j-1, \quad \chi_{1}=\left(\left|a^{(1)}+1\right|\right)\left(\left|a^{(2)}\right|+\sum_{i=1}^{p}\left|k_{i}\right|-p+|b|+|\alpha|+\right.$ $\left.\sum_{j=1}^{q}\left|\omega_{j}\right|+q+|\beta|\right)$ and $\chi_{2}=|a|+\sum_{i=1}^{p}\left|k_{i}\right|=\epsilon_{p}$.

To prove the proposition, it is enough to show that the following two statements hold:

- The complex $C(K, \Omega ; \phi)$ models the gauge bundle $P \times_{A d} G$ (in particular, its cohomology is isomorphic to the cohomology of the gauge bundle).

- Complexes $C(K, \Omega ; \phi)$ and $K \hat{\otimes}_{\phi} \Omega$ are homotopy equivalent.

First, let us calculate the cohomology of $C(K, \Omega ; \phi)$. Let $\Omega_{\phi} \otimes K$ be the left-twisted tensor product of $K$ and $\Omega$, i.e. $\Omega_{\phi} \otimes K \cong \Omega \otimes K$ as linear spaces, and the differential is

$$
{ }_{\phi} d(\omega \otimes k)=d \omega \otimes k+(-1)^{|\omega|}\left(\omega \otimes d k+\omega \phi\left(k^{(1)}\right) \otimes k^{(2)}\right) .
$$

It is clear that $\Omega_{\phi} \otimes K$ is a model for the cohomology of the principal bundle $\hat{P}=P \times_{R^{-1}} G$, where $R^{-1}$ is the left action of $G$ on itself, given by $R_{g}^{-1}(h)=h g^{-1}$. (One can use the formulae for $\phi$ given in this article to show it.) Observe, that $\hat{P}$ is diffeomorphic to $P$ as a differentialble manifold, while the structure group $G$ acts on $\hat{P}$ from the right (we assumed that it acted on $P$ from the left). It is now easy to see that $C(K, \Omega ; \phi)$ is homotopy-equvalent to the realization of the cosimplicial simplicial $D G$ algebra $\Omega_{D R}\left(\mathscr{C}_{\bullet}(G, X ; P)\right)$, where $\mathscr{C}_{\bullet}(G, X ; P)$ is the simplicial cosimpli- 
cial manifold

$$
\mathscr{C}_{p, q}(G, X ; P)=\hat{P} \times \underbrace{G \times \ldots \times G}_{p} \times P \times \underbrace{X \times \ldots \times X}_{q} .
$$

Here $p$ denotes the simplicial, and $q$ is the cosimplicial dimension of this space. The simplicial structure maps are given by the usual bar-construction morphisms, so that for a fixed $q$ we obtain $B_{\bullet}(\hat{P}, G, P) \times X^{\times q}$, and the coface (resp. codegeneracy) maps will be given by diagonal embedings (resp. projections) in the cartesian powers of $X$. According to the Bott-Segal theorem (see [4]), cohomology of $C(K, \Omega ; \phi)$ is isomorphic to the cohomology of the realization of $\mathscr{C}_{\bullet}(G, X ; P)$. On the other hand, realizing this space first in the simplicial, and then in the cosimplicial direction, and using the fact that $P$ is a free $G$-space we obtain first the cosimplicial space

$$
\mathscr{F}_{q}(P, X, \hat{P}) / G=(P \times \underbrace{X \times \ldots \times X}_{q} \times \hat{P}) / \sim,
$$

where the relation $\sim$ is given by $\left(g \cdot p, x_{1} \ldots, x_{q}, \hat{p}\right) \sim\left(p, x_{1}, \ldots, x_{q}, \hat{p} \cdot g\right)$. Since the action of $G$ is free, we can commute the geometric realization and the factorization procedure, thus from Anderson's theorem, see [1], it follows that

$$
\left|\mathscr{C}_{\bullet}(G, X ; P)\right|=\mathscr{P}\left(X ; \hat{P} \times_{G} P\right) .
$$

Here $\hat{P} \times_{G} P=\{(\hat{p}, p)\} /(\hat{p} \cdot g, p) \sim(\hat{p}, g \cdot p)$. If $\pi_{1}, \pi_{2}$ are the natural projections of $\hat{P} \times{ }_{G} P$ on $X$, then

$$
\mathscr{P}\left(X ; \hat{P} \times_{G} P\right)=\left\{(\gamma,[\hat{p}, p]) \mid \gamma:[0 ; 1] \rightarrow X, \gamma(0)=\pi_{1}([\hat{p}, p]), \gamma(1)=\pi_{2}([\hat{p}, p])\right\} .
$$

Now it is enough to observe that $\mathscr{P}\left(X ; \hat{P} \times{ }_{G} P\right)$ is homotopic to the preimage of the diagonal in $X \times X$ under the pojection $\pi_{1} \times \pi_{2}: \hat{P} \times{ }_{G} P \rightarrow X \times X$, which is isomorphic to $P \times{ }_{A d} G$.

In order to prove the second statement, let us consider the complex $F(K, \Omega ; \phi)$ :

$$
F(K, \Omega ; \phi)=\bigoplus_{p} K \otimes \bar{K}^{\otimes p} \otimes K \otimes A,
$$

with differential

$$
\begin{gathered}
d\left(a\left[k_{1}|\ldots| k_{p}\right] b \otimes \omega\right)=d a\left[k_{1}|\ldots| k_{p}\right] b \otimes \omega+\sum_{i=1}^{p}(-1)^{\epsilon_{i}} a\left[k_{1}|\ldots| d k_{i}|\ldots| k_{p}\right] \otimes \omega \\
+(-1)^{\epsilon_{p}} a\left[k_{1}|\ldots| k_{p}\right] d b \otimes \omega+(-1)^{\epsilon} a\left[k_{1}|\ldots| k_{p}\right] b \otimes d \omega+a^{(1)}\left[a^{(2)}\left|k_{1}\right| \ldots \mid k_{p}\right] b \otimes \omega \\
+\sum_{i=1}^{p}(-1)^{\epsilon_{i}} a\left[k_{1}|\ldots| k_{i}^{(1)}\left|k_{i}^{(2)}\right| \ldots \mid k_{p}\right] b \otimes \omega+(-1)^{\epsilon_{p}} a\left[k_{1}|\ldots| k_{p} \mid b^{(1)}\right] b^{(2)} \otimes \omega \\
+(-1)^{\epsilon_{p}} a\left[k_{1}|\ldots| k_{p}\right] b^{(1)} \otimes \phi\left(b^{(2)}\right) \omega+(-1)^{\chi_{3}} a^{(2)}\left[k_{1}|\ldots| k_{p}\right] b \otimes \omega \phi\left(a^{(1)}\right) . \\
-345-
\end{gathered}
$$


Here all the gradings, signs and notation are taken from the definition of $C(K, \Omega ; \phi)$, and $\chi_{3}=\left(\left|a^{(1)}\right|+1\right)\left(\left|a^{2)}\right|+\sum_{i=1}^{p}\left|k_{i}\right|-p+|b|+|\omega|\right)$. There is a chain map $C(K, \Omega ; \phi) \rightarrow F(K, \Omega ; \phi)$, given by projection of $C(K, \Omega ; \phi)$ onto the $q=0$ part and multiplication $\Omega \otimes \Omega \rightarrow \Omega$. We claim, that this map induces an isomorphism in cohomology: for proof introduce filtrations on both complexes, by degree $q$ in $C(K, \Omega ; \phi)$ and a constant filtration in $F(K, \Omega ; \phi)$. The map we consider respects these filtrations and induces an isomorphism at the first stage of the spectral sequences. Recall, that we assumed, that $\Omega$ has unit, hence its bar-resolution is contractible.

Finally, there's a map $K \hat{\otimes}_{\phi} \Omega \rightarrow F(K, \Omega ; \phi)$, given by comultiplication in $K$ followed by embedding with $p=0$. By similar arguments, involving spectral sequences, one proves that this embedding induces an isomorphism in cohomology.

If $\Omega$ is not connected, it is necessary to consider the normalized variants of the complexes $C(K, \Omega ; \phi)$, etc., i.e. take quotients by subcomplexes, generated by the 0 -dimensional part of $\Omega$. Since we assume that the base $X$ is 1-connected and $\Omega$ is a model for $X$, it follows that $\Omega$ is homotopyequivalent to a connected algebra, and hence the 0 -dimensional part of $\Omega$ can be dispensed with without any trouble.

One can show that if $\phi$ and $\phi^{\prime}$ are gauge equivalent twisting cochains and $c: K \rightarrow \Omega$ is an invertible map, that establishes this equivalence, then the corresponding bitwisted tensor products are isomorphic; the isomorphism is given by the map

$$
\begin{aligned}
& \hat{c}: K \hat{\otimes}_{\phi} \Omega \rightarrow K \hat{\otimes}_{\phi^{\prime}} \Omega \\
& k \otimes \omega \mapsto(-1)^{\left(\left|k^{(1)}\right|+1\right)\left(\left|k^{(2)}\right|+\left|k^{(3)}\right|+|\omega|\right)} k^{(2)} \otimes c\left(k^{(3)}\right) \omega c^{-1}\left(k^{(1)}\right),
\end{aligned}
$$

the inverse of $\hat{c}$ is equal to $\widehat{c^{-1}}$. Besides this, every $A_{\infty}$-map $\mathcal{P}: \Omega \rightarrow \Omega^{\prime}$ induces the map of the bitwisted tensor products:

$$
\begin{array}{r}
(1 \hat{\otimes} \mathcal{P})(k \otimes a)=\sum_{n \geqslant 1} \sum_{m=1}^{n} k^{(m)} \otimes \mathcal{P}(n)\left(\phi\left(k^{(m+1)}\right) \otimes\right. \\
\left.\ldots \otimes \phi\left(k^{(n)}\right) \otimes a \otimes \phi\left(k^{(1)}\right) \otimes \ldots \otimes \phi\left(k^{(m-1)}\right)\right) .
\end{array}
$$

Finally, consider the formula

$$
k \otimes \omega \mapsto \sum_{n}\left[\phi\left(k^{(1)}\right)|\ldots| \phi\left(k^{(n)}\right)\right] \omega,
$$

we use $\mid$ instead of $\otimes$. It determines an analogue of homological monodromy map

$$
\begin{gathered}
\tilde{\hat{\phi}}: K \hat{\otimes}_{\phi} \Omega \rightarrow N H(\Omega) . \\
-346-
\end{gathered}
$$


It is evident that maps $\tilde{\hat{\phi}}$ and $\tilde{\hat{\phi}^{\prime}}$, induced by gauge-equivalent twisting cochains are homotopic. The corresponding chain homotopy is given by

$$
\begin{gathered}
\hat{H}(k \otimes a)=\sum_{n=0}^{\infty} \sum_{l=1}^{n-1} \phi\left(k^{(1)}\right) \otimes \ldots \otimes \phi\left(k^{(l-1)}\right) \otimes c^{-1}\left(k^{(l)}\right) \otimes \phi^{\prime}\left(k^{(l+1)}\right)(3.3 \\
\otimes \ldots \otimes \phi^{\prime}\left(k^{(n-1)}\right) \otimes c\left(k^{(n)}\right) a .
\end{gathered}
$$

One can introduce more structure on complexes $K \hat{\otimes}_{\phi} \Omega$ and $N H(\Omega)$. Recall that the gauge bundle $P \times_{A d} G$ is a bundle of groups, so one can define a product on global sections of $P \times A d$. Geometrically, this structure is determined by the map

$$
\left(P \times_{A d} G\right) \times_{X}\left(P \times_{A d} G\right) \rightarrow P \times_{A d} G,
$$

verifying evident associativity conditions. Similar map can be defined for the free loop space $\mathcal{L} X$ of $X$, when we regard it as a fibre bundle over $X$ with respect to the evaluation map $e(\gamma)=\gamma(0)$, the associativity is replaced by homotopy associativity properties. To find an analog of this map on the algebraic level, we first of all demand that the model $A$ of $P \times{ }_{A d} G$ should be a DG module over an algebra $\Omega$, corresponding to $X$, and second, that there be a map

$$
A \rightarrow A \otimes_{\Omega} A,
$$

verifying the usual coassociativity conditions, and similarly for the model of $\mathcal{L} X$. More generally one can speak about homotopy analogs of all these maps, i.e. about the $A_{\infty}$-algebras and coalgebras, their modules etc., but this will bring us far beyond the modest purposes of the present paper. However, one can easily introduce the necessary maps on $K \hat{\otimes}_{\phi} \Omega$ and $N H(\Omega)$, when $\Omega$ is commutative. Namely we put, respectively,

$$
k \hat{\otimes} \omega \mapsto(-1)^{\left|k^{(2)}\right||\omega|}\left(k^{(1)} \hat{\otimes} \omega\right) \otimes_{\Omega}\left(k^{(2)} \hat{\otimes} 1\right)
$$

and

$$
\left[\omega_{1}|\ldots| \omega_{n}\right] a \mapsto \sum_{i=0}^{n}(-1)^{\eta_{i}}\left(\left[\omega_{1}|\ldots| \omega_{i}\right] a\right) \otimes_{\Omega}\left(\left[\omega_{i+1}|\ldots| \omega_{n}\right] 1\right) .
$$

Here $\eta_{i}=|a|\left(\left|\omega_{i+1}\right|+\ldots+\left|\omega_{n}\right|+n-i\right)$ and we let $\Omega$ act on $K \hat{\otimes}_{\phi} \Omega$ by multiplication in the $\Omega$, and on $N H(\Omega)$ by multiplication in the last tensor place. In case, when $\Omega$ is not commutative and only homotopy-commutative, one should use the higher homotopy maps. Also if $\Omega$ and $\Omega^{\prime}$ are homotopyequivalent algebras, one can use the $A_{\infty}$-map $\mathcal{P}: \Omega \rightarrow \Omega^{\prime}$, which establishes this quasi-isomorphism, to determine a new twisting cochain $\mathcal{P}(\phi): K \rightarrow \Omega^{\prime}$, 
corresponding to $\phi$ under the homotopy equivalence. Thus we can always assume that $\Omega$ is commutative. It is now a matter of simple calculation to show that the map $\tilde{\hat{\phi}}$ intertwines the coproduct structures (3.37) and (3.38) and that, if $\phi, \phi^{\prime}$ are gauge equivalent via $c$, and $\Omega$ is commutative, then the map $\hat{c},(3.34)$, commutes with the coproducts.

\section{Getzler-Jones-Petrack's map and Bismut's class}

In this section we shall explain in what sense the cyclic Chern class of Getzler, Jones and Petrack, Bismut and others (see [11], [2] and [27] and references therein) are related to constructions, described in the previous sections. To this end we shall need to make a couple of intermediate steps, which connect the twisting cochains and the chain maps of the previous sections to the Getzler, Jones and Petrack's results. We concentrate on the case when the principal bundle is equal to the frame bundle of a vector bundle. Then one can choose connection in a very special way, which allows us work with the bundle as if it were trivial. In this way we obtain the Getzler-Jones-Petrack map, as a variant of the homological monodromy map. We show, that this map is homotopic to $\tilde{\hat{\phi}},(3.35)$, associated with a twisting cochain. Finally, we give an $S^{1}$-equivariant version of this theory, which allows one treat the Bismut's class (or cyclic Chern character).

\subsection{The Getzler-Jones-Petrack map}

Let $E$ be a rank $n$ complex vector bundle over a compact manifold $X$. One can associate to it the principal $G L(n)$-bundle $P_{E}$ of frames in $E$, so that $E=P_{E} \times_{G L(n)} \mathbb{C}^{n}$. One can apply to $P_{E}$ the methods, described in previous sections and get formulae for the twisting cochain, for the characteristic classes, etc.. In particular, one can obtain the map

$$
\tilde{\hat{\phi}}: K \hat{\otimes}_{\phi} \Omega \rightarrow N H^{*}(\Omega),
$$

where $K$ is a DG coalgebra modeling $G L_{n}$ and $\Omega$ a DG algebra modeling $X$, see proposition 3.3. For example, one can take $K=\Omega_{\text {poly }}\left(G L_{n}\right)$ and $\Omega=$ $\check{C}\left(\mathcal{U}, \Omega_{D R}(U)\right)$. Using the glueing construction, described in the paragraph 2.4 , one can substitute $\Omega_{D R}(X)$ for $\check{C}\left(\mathcal{U}, \Omega_{D R}(U)\right)$, and the domain of $\tilde{\hat{\phi}}$ can be replaced with the quasi-equivalent complex $\Omega_{D R}\left(\tilde{P}_{E}\right)$, where $\tilde{P}_{E}$ is the gauge bundle of $P_{E}$ (we use this notation instead $\left(P_{E}\right)_{A d}$ for the sake of brevity).

On the other hand, the fact, that $P_{E}$ is associated with a vector bundle can be used to obtain another map with the same domain and range, given 
in pretty explicit terms: let $E \leftrightarrows X \times \mathbb{C}^{N}$ be an inclusion of/projection on $E$ to/from a trivial bundle. Let $p \in \operatorname{Mat}_{N}\left(C^{\infty}(X)\right)$ be the corresponding projector. We shall use $p$ to construct the morphism we need. Let $X \times G L(N)$ be the gauge bundle of the trivial vector bundle $N=X \times \mathbb{C}^{N}$. Since $E \subseteq N$, we can regard $\tilde{P}_{E}$ as a subbundle of $X \times G L(N)$. Namely denote by $q$ the complementary projection $q=1-p$, so that $p q=q p=0$ and $p+q=1$. Then $\tilde{P}_{E}$ is equal to the following subbundle of $X \times G L(N)$ :

$\check{P}_{E}=\left\{(x, g) \in X \times G L(N) \mid g(\operatorname{Im} p(x))=\operatorname{Im} p(x), g(\operatorname{Im} q(x))=1_{\operatorname{Im} q(x)}\right\}$.

Equivalently, the latter condition can be written in the matrix form as follows: $g q(x)=q(x) g=q(x)$. Let us denote the complementary subbundle by $\bar{E}$

In the terms of functions and differential forms on the bundles, this inclusion of gauge bundles induces a restriction morphism $\Omega_{D R}(X \times G L(N)) \rightarrow$ $\Omega_{D R}\left(\tilde{P}_{E}\right)$, which is epimorphic (it follows from local considerations). To render this and following constructions rigoruos, one should consider the algebras of vertically-polynomial differential forms $\Omega_{v p o l y}^{*}\left(\tilde{P}_{E}\right), \Omega_{v p o l y}^{*}(X \times$ $G L(N))$ etc., see section 3.4, i.e. all the forms must be from $\Omega_{\text {poly }}(G)$ on each fibre. This is what we shall assume now, although we shall not encumber our text with redundant notations. One can now regard $\Omega_{D R}\left(\tilde{P}_{E}\right)$ as the factor-algebra of $\Omega_{D R}(X \times G L(N))$ modulo the kernel of the restriction. On the other hand one can describe the generating relations of this kernel. We obtain a presentation of $\Omega_{D R}\left(\tilde{P}_{E}\right)$ as factor-algebra of $\Omega_{D R}(X \times G L(N))$ by the DG ideal generated by the functions

$$
\sum_{j} u_{i j} p_{j k}-u_{i k}, \sum_{j} p_{i j} u_{j k}-p_{i k}, i, k=1, \ldots, N .
$$

There is an alternative point of view on this construction, which might make it easier for understanding: consider the diagramm of group bundles:

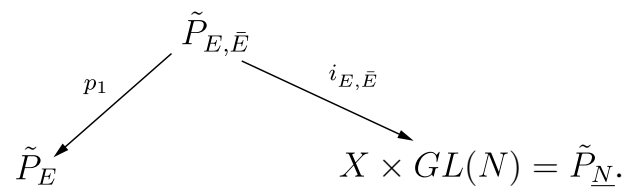

Here $\tilde{P}_{E, \bar{E}}=\tilde{P}_{E} \times_{X} \tilde{P}_{\bar{E}}, p_{1}$ is the projection on the first factor, and $i_{E, \bar{E}}$ is the natural embedding of $\tilde{P}_{E, \bar{E}}$ into $\tilde{P}_{N}$, induced by the embeddings of $E$ and $\bar{E}$ into $\underline{N}$. The left leg of this diagramm is a fibration, and the right one - an inclusion. Then one can regard the functions on $\tilde{P}_{E}$ as a function on $\tilde{P}_{\underline{N}}$ whose restriction to $\tilde{P}_{E, \bar{E}}$ doesn't depend on coordinates in the $\bar{E}$ directions. 
Recall that when we embed $E \rightarrow \underline{N}$, we can find global expressions, representing a connection form on $E$ and its curvature (see [11]):

$$
A=p d p-(1-p) d(1-p), F=p d p d p
$$

They are $\operatorname{Mat}_{N}(\mathbb{C})$-valued differential 1- and 2-forms on $X$, such that $A_{x, \xi}(\operatorname{Im} p(x)) \subseteq \operatorname{Im} p(x)$ and $F_{x, \xi, \eta}(\operatorname{Im} p(x)) \subseteq \operatorname{Im} p(x)$ for all $x \in X$ and all $\xi, \eta \in T_{x} X$. On the other hand, regarding $\operatorname{Mat}_{N}(\mathbb{C})=\mathfrak{g l}_{N}$ as the space of left-invariand vector fields on $G L_{N}$, so the elements of $\mathfrak{g l}_{N}$ are derivatives in $\Omega_{D R}(G L(N))$, (or on $\Omega_{D R}(X \times G L(N)) \cong \Omega_{D R}(X) \otimes \Omega_{D R}(G L(N))$, if we regard elements of the Lie algebra as vertical vector fields), we can consider the map

$$
\begin{aligned}
& \phi_{E}: \Omega_{D R}(X) \otimes \Omega_{D R}(G L(N)) \rightarrow \Omega_{D R}(X) \otimes \Omega_{D R}(X) \otimes \Omega_{D R}(G L(N)), \\
& \phi_{E}(\alpha \otimes \beta)=\alpha \otimes\left\{\sum_{i j} A_{i j} \otimes X_{i j}(\beta)+F_{i j} \otimes I_{i j}(\beta)\right\} .
\end{aligned}
$$

Here $\left\{X_{i j}\right\}$ is a basis of $\mathfrak{g l}_{N}$ (derivatives in $\Omega_{D R}(G L(N))$ as explained above), and $\left\{I_{i j}\right\}$ are the contractions with corresponding vector fields. The following proposition is simple, but important for the future discussion.

Proposition 4.1. - One can restrict $\phi_{E}$ to the factor-algebra $\Omega_{D R}\left(\tilde{P}_{E}\right)=\Omega_{D R}(X \times G L(N)) / I$, where $I$ is the ideal, generated by functions (4.1).

Proof. - It is enough to check, that derivations $\sum_{i j} A_{i j} \otimes X_{i j}, \sum_{i j} F_{i j}$ $\otimes I_{i j}$ vanish on the kernel of the factorisation $\Omega_{D R}(X \times G L(N)) \rightarrow \Omega_{D R}\left(\tilde{P}_{E}\right)$. But this is clear since the kernel is generated by the matrix elements of $1_{N}-p(x)$.

One can use the restriction of $\phi_{E}$ to $\Omega_{D R}\left(\tilde{P}_{E}\right)$ to define a map

$$
\begin{aligned}
& \tilde{\phi}_{E}: \Omega_{D R}\left(\tilde{P}_{E}\right) \rightarrow N H\left(\Omega_{D R}(X)\right), \\
& \tilde{\phi}_{E}(a)=\left.\sum_{n \geqslant 0} \phi_{E}^{\otimes n}(a)\right|_{1_{G L(N)} .} .
\end{aligned}
$$

Here $a$ is an arbitrary differential form on $\tilde{P}_{E}, \phi_{E}^{\otimes n}$ is the $n$-fold iteration of $\phi_{E}$. We shall call $\tilde{\phi}_{E}$ the Getzler-Jones-Petrack (GJP) map. As a matter of fact, in the genuine construction of [11], one needs to introduce additional equivariant structure. We shall discuss this refinement in section 4.3. Now let us discuss the algebraic properties of the map $\tilde{\phi}_{E}$. 
Proposition 4.2. - The map $\tilde{\phi}_{E}$ commutes with differentials. This map is a homomorphism of DG algebras with respect to the usual multiplication in $\Omega_{D R}\left(\tilde{P}_{E}\right)$ and the cyclic shuffle product in $N H\left(\Omega_{D R}(X)\right)$. The change of projection $p$ that determines $\tilde{\phi}_{E}$, acts on $\tilde{\phi}_{E}$ by the conjugation of its domain by a section of the gauge bundle.

Proof. - All the properties follow from the results of sections 3.2 and 3.3. To prove the last statement observe that $\tilde{\phi}_{E}$ is just the gauge bundle monodromy map $\widetilde{T}_{A}$, see section 3.4 .

Consider the map

$$
\Omega_{D R}\left(\tilde{P}_{E}\right) \rightarrow \Omega_{D R}\left(\tilde{P}_{E}\right) \otimes_{\Omega_{D R}(X)} \Omega_{D R}\left(\tilde{P}_{E}\right),
$$

induced by the comultiplication in the algebra $\Omega_{D R}(G L(N))$ : the ideal $I$ (see (4.1)) is in fact a coideal, which follows from the equation $p^{2}=p$. Then one can show (compare the discussion of the map $\widetilde{M}_{A}$ ) that $\tilde{\phi}_{E}$ intertwines the comultiplications (4.3) and $N H\left(\Omega_{D R}(X)\right) \rightarrow N H\left(\Omega_{D R}(X)\right) \otimes_{\Omega_{D R}(X)}$ $N H\left(\Omega_{D R}(X)\right)$, given by (3.38).

\subsection{The comparison theorem}

The main purpose of this section is to prove the theorem

Theorem 4.3. - Under the identifications we made, the GJP map $\tilde{\phi}_{E}$ is chain homotopic to $\tilde{\hat{\phi}}$.

Proof. - Before we prove this statement, we should explain, which identifications we use here. First, if $\phi$ is a twisting cochain on $K$ with values in $\Omega, \tilde{\hat{\phi}}$ will denote the map $K \hat{\otimes}_{\phi} \Omega \rightarrow N H(\Omega)$ (see (3.35)), determined by these data. In our case we take $K=\Omega_{G}$ for $G=G L(n ; \mathbb{C})$ and $\Omega=\Omega_{D R}(X)$ or $\left|\Omega_{D R}\left(\mathcal{N U}_{*}\right)\right|$ (we choose these DG algebras for the sake of their commutativity and also because $\tilde{\phi}_{E}$ takes values in $\left.N H\left(\Omega_{D R}(X)\right)\right)$ and let $\phi$ be the twisting cochain on $\Omega_{G}$ with values in $\Omega$. To this end we need to start with the twisting cochain $\phi_{P}$ or $\xi$ (sections 2.1, 2.3), with values in Čech complex and use the higher homotopy maps, as described in section 2.4 to pass from the Čech cochains to differential forms. It follows from the homotopy equivalence of all the constructions that this theorem doesn't depend on the choice of $\Omega$ and $K$, so the maps $\tilde{\hat{\phi}}$ are homotopic to $\tilde{\phi}_{E}$ for all $\Omega$ and $K$.

Second, we use the homotopy equivalence of the proposition 3.3 that relates the complexes $K \hat{\otimes}_{\phi} \Omega$ and $\Omega_{D R}\left(\tilde{P}_{E}\right)$ where $E$ is the vector bundle associated with $P$ by the canonical $n$-dimensional representation of $G L(n ; \mathbb{C})$. 
Then $P \times_{A d} G L(n ; \mathbb{C})=\tilde{P}_{E}$, while $P$ is equal to the frame bundle $P_{E}$ of $E$. In fact, we shall even show that the equivalence of 3.3 in our case intertwines coproducts, given by (4.3) and (3.37), so that $\tilde{\hat{\phi}}$ and $\tilde{\phi}_{E}$ will be homotopic as homomorphisms of coalgebras. So let us first prove that $\Omega_{D R}\left(\tilde{P}_{E}\right)$ and $\Omega_{G} \hat{\otimes}_{\phi} \Omega_{D R}(X)$ are homotopic as coalgebras over $\Omega_{D R}(X)$. To this end, consider two intermediate objects: first the groupoid $G P=\hat{P} \times{ }_{G} P$ (see the definition of $\hat{P}$ in the proof of 3.3; here and further in this section $G$ will denote the group $G L(n ; \mathbb{C})$ ). Source and target maps $s, r: \mathscr{P} \rightarrow X$ are given by the projection on the first and the second factor respectively, and the composition is given by the following rule:

$$
(u, v) *(x, y)=(u g, y),
$$

for all $u, x \in \hat{P}, v, y \in P$ such that $\pi(v)=\hat{\pi}(x)$ and $g=g_{1} g_{2}$, where $\varphi_{\alpha}(v)=\left(g_{1}, \pi(v)\right), \hat{\varphi}_{\alpha}(x)=\left(\hat{\pi}(x), g_{2}\right)\left(\varphi_{\alpha}, \hat{\varphi}_{\alpha}\right.$ are the local trivializations in $P$ and $\hat{P}$ respectively.) Then $P \times_{A d} G=\tilde{P}_{E}$ can be embedded to $G P$ as a subbundle above the diagonal of $X \times X$.

The second object we shall consider here is an algebraic analog of $G P$, the complex $G P=G P(\Omega, K)=\Omega_{\phi} \otimes K \otimes_{\phi} \Omega$. It is equal to $\Omega \otimes K \otimes \Omega$ as a vector space, while the differential ${ }_{\phi} d_{\phi}$ is given by the formula (we omit the tensor signs for the sake of brevity)

$$
\begin{gathered}
{ }_{\phi} d_{\phi}(a k b)=d a k b+(-1)^{|a|}\left(a d k b+a \phi\left(k^{(1)}\right) k^{(2)} b\right) \\
+(-1)^{|a|+|k|}\left(a k d b+(-1)^{\left|k^{(2)}\right|} a k^{(1)} \phi\left(k^{(2)}\right) b\right) .
\end{gathered}
$$

This complex is a bimodule over $\Omega$ with respect to the left and the right multiplication on the left and right tensor legs respectively; there is a map $G P \rightarrow G P \otimes_{\Omega} G P$, given by

$$
a k b \mapsto\left(a k^{(1)} 1\right) \otimes_{\Omega}\left(1 k^{(2)} b\right) .
$$

Our first claim is that $G P$ is a model for $G P$, i.e. we claim that there is a quasi-equivalence $\mathscr{I}$ of $G P$ and the de Rham algebra of $\mathscr{Y} P$ regarded as coalgebras over $\Omega$, i.e. this quasi-isomorphism intertwines the "coproducts" described above. The first statement, i.e. that the complexes are quasiequivalent, follows from the isomorphism

$$
G P=\left(\Omega_{\phi} \otimes K\right) \otimes^{K}\left(K \otimes_{\phi} \Omega\right),
$$

where $\otimes^{K}$ is the cotensor product over the coalgebra $K$. Recall that $K \otimes_{\phi} \Omega$ is a model of $P$ as $G$-space. Besides this, since the action coaction of $K$ is free, the cotensor product over $K$ and homotopy tensor product over $K$ are equivalent and we can apply the reasoning we used in the proof of 3.3. Thus, 
we obtain a quasi-isomophism of these complexes that we shall denote by $\mathscr{S}$. Observe, that the quasi-isomorphism $\mathscr{J}$ of proposition 3.3 is restriction of $\mathscr{I}$ onto the diagonal.

Next we prove that $\mathscr{I}$ commutes with the coaction. To this end observe that $G P \otimes_{\Omega} G P$ is a model of $G P \times_{X} G P$ and, since the maps

$$
k \otimes a \mapsto k^{(1)} \otimes\left(k^{(2)} \otimes a\right) \text { and } a \otimes k \mapsto\left(a \otimes k^{(1)}\right) \otimes k^{(2)}
$$

correspond under the identifications of theorem 3.3, to the action of $G$ on $P$ and $\hat{P}$ respectively, and these two actions determine the groupoid structure on $\mathscr{G} P$, we conclude that $\mathscr{I}$ is a map of coalgebras over $\Omega$.

Let now $\Omega=\Omega_{D R}(X)$, then $\Omega_{G} \hat{\otimes}_{\phi} \Omega_{D R}(X)$ embeds into $G P$ diagonally as a coalgebra over $\Omega_{D R}(X)$ : we just send $a k b$ to $(-1)^{|a||k|} k \otimes a b$. We shall denote this map by $\tilde{m}^{*}$. This embedding, on the one hand, intertwines the coproducts on both sides, and on the other hand, it makes the following diagram commute:

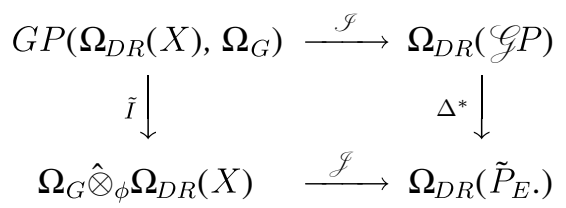

Since the both vertical, and the top horizontal arrows commute with the coproducts, and the vertical maps are onto, we conclude that the bottom arrow also commutes with coproduct.

Now we can prove that $\tilde{\phi}_{E}$ is homotopic to $\tilde{\hat{\phi}}$. Observe, that the homotopy equivalence of proposition 3.3 can be constructed as follows: take the space

$\mathscr{P}\left(X ; \hat{P} \times_{G} P\right)=\left\{(\gamma,[\hat{p}, p]) \mid \gamma:[0 ; 1] \rightarrow X, \gamma(0)=\pi_{1}([\hat{p}, p]), \gamma(1)=\pi_{2}([\hat{p}, p])\right\}$,

then this space is homotopy equivalent to $\hat{P}_{E}$, the homotopy being given by raising to $\mathscr{P}\left(X ; \hat{P} \times_{G} P\right)$ the homotopy contracting the free path space of a topological space. On the other hand, its cohomology is computed by the complex

$$
\Omega_{D R}\left(\hat{P} \times_{G} P\right) \otimes_{\Omega_{D R}(X)^{e v}} \bar{B}\left(\Omega_{D R}(X), \Omega_{D R}(X), \Omega_{D R}(X)\right) .
$$

Here for an algebra $A, A^{e v}=A \otimes A^{o} ; A^{o}$ denotes $A$ with inverted multiplication: $a \circ b=(-1)^{|a||b|} b a$. There's an evident generalization of the Chen's iterated integral map, [8], to a map $\Omega_{D R}\left(\hat{P} \times_{G} P\right) \otimes_{\Omega_{D R}(X)^{e v}} B\left(\Omega_{D R}(X)\right), \rightarrow$ 
$\Omega_{D R}\left(\mathscr{P}\left(X ; \hat{P} \times_{G} P\right)\right)$; to construct this map one should regard $\mathscr{P}\left(X ; \hat{P} \times_{G}\right.$ $P)$ as a pullback:

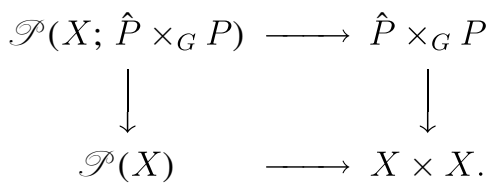

And instead of $\Omega_{D R}\left(\hat{P} \times_{G} P\right)$ one can use $G P\left(\Omega_{D R}(X), \Omega_{D R}(G L(n))\right)$.

In order to construct the homotopy equivalence $\mathscr{P}\left(X ; \hat{P} \times_{G} P\right) \leftrightarrows \tilde{P}_{E}$, we interprete the groupoid $\mathscr{G} P=\hat{P} \times{ }_{G} P$ as follows: for any pair of points $\left(x, x^{\prime}\right) \in X \times X, \quad P_{\left(x, x^{\prime}\right)}=I s o\left(E_{x}, E_{x^{\prime}}\right)$, the space of linear isomorphisms between the fibres of $E$ over $x$ and $x^{\prime}$. We shall often denote this groupoid just by $\mathscr{G}_{E}$. Let $A$ be a connection in $E$, for example, a Grassmanian connection, induced by an embedding of $E$ into a trivial bundle. Then the homotopy equivalence is induced by the maps:

$$
\Phi: \tilde{P}_{E} \rightarrow \mathscr{P}\left(X ; \hat{P} \times_{G} P\right), \quad i(a)=\left(*_{\pi(a)},[\hat{p}, p]\right)
$$

where $\pi: \tilde{P}_{E} \rightarrow X$ is the projection, $*_{x}$ for $\pi(a)=x \in X$ is a constant path $*_{x}(t)=x, t \in[0 ; 1]$, and $\hat{p} \in \hat{P}, p \in P$ are defined as the elements in $\hat{P}_{\pi(a)}$ and $P_{\pi(a)}$ respectively, which in the corresponding local charts $\hat{\varphi}_{\alpha}, \varphi_{\alpha}$ of $\hat{P}$ and $P$ are represented by such elements $(\pi(a), \hat{h})$ and $(h, \pi(a))$, that $\hat{h} h=g$ where $\tilde{\varphi}_{\alpha}(a)=(\pi(a), g)$. Its homotopy inverse is given by

$$
\Psi: \mathscr{P}\left(X ; \hat{P} \times_{G} P\right) \rightarrow \tilde{P}_{E}, \quad p(\gamma,[\hat{p}, p])(e)=\left(\int_{\gamma} A\right)^{-1}[\hat{p}, p](e)
$$

where $e$ is an arbitrary element in the fibre of $E$, which lies above $s([\hat{p}, p])$ (recall that $\left.\hat{P} \times{ }_{G} P=G P\right), \int_{\gamma} A$ is the parallel translation in $E$ along the path $\gamma$ with respect to the connection $A$, and $[\hat{p}, p](e)$ is the action of $\mathscr{P} P$ on $E$. Clearly, $\Psi \Phi=I d_{\tilde{P}_{E}}$. On the other hand, $\Phi \Psi$ is homotopic to the identity via the homotopy $H_{s}, s \in[0 ; 1]$, given by

$H_{s}: \mathscr{P}\left(X ; \hat{P} \times_{G} P\right) \rightarrow \mathscr{P}\left(X ; \hat{P} \times_{G} P\right) \quad H_{s}(\gamma,[\hat{p}, p])=\left(\gamma_{s}^{\prime},\left(\int_{\gamma_{s}^{\prime \prime}} A\right)^{-1}[\hat{p}, p]\right)$.

Here $\gamma_{s}^{\prime}(t)=\gamma(s t)$ and $\gamma_{s}^{\prime \prime}(t)=\gamma(s+(1-s) t)$ for $s, t \in[0 ; 1]$ and we regard $\mathscr{G} P$ as $\mathscr{G}_{E}$.

The purpose of these considerations is in the following remark: one can write down an explicit formula for the inverse image map, induced by $\Psi$, between the de Rham complex of $\tilde{P}_{E}$ and a model for $\mathscr{P}\left(X ; \hat{P} \times_{G} P\right)$. To this end observe that there is a map $M: \mathscr{G}_{E} \times_{X}{ }^{o p} \mathscr{G}_{E} \rightarrow \tilde{P}_{E}$, where

$$
\mathscr{G}_{E} \times_{X}{ }^{o p} \mathscr{G}_{E}=\left\{(a, b) \in \mathscr{G}_{E} \times \mathscr{G}_{E} \mid r(a)=s(b), r(b)=s(a)\right\},
$$


(we shall use similar notation below), $M$ is composition of $a$ and $b$. Next consider the embedding of $E$ into a trivial bundle $\underline{N}=X \times \mathbb{C}^{N}$. Let $p$ be the projection $X \times \mathbb{C}^{N} \rightarrow E$, and $q=1-p$ be the complementary projection, $\bar{E}=\operatorname{Im}(1-p)$. Then there exists an evident diagram of groupoids, associated with the bundles $E, F$ and $\underline{N}$ (compare it with the diagram $(4.2))$ :

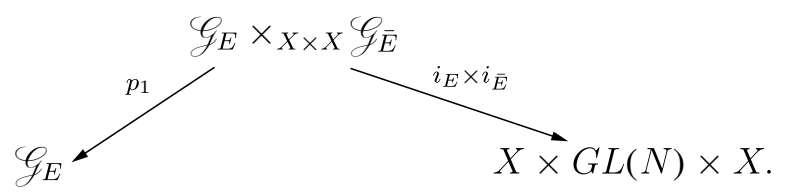

Here $\mathscr{G}_{E} \times{ }_{X \times X} \mathscr{G}_{\bar{E}}$ is the fibre product of $\mathscr{G}_{E}$ and $\mathscr{G}_{\bar{E}}$ over $X \times X$ :

$$
\mathscr{G}_{E} \times{ }_{X \times X} \mathscr{G}_{\bar{E}}=\left\{(e, f) \in \mathscr{G}_{E} \times \mathscr{G}_{\bar{E}} \mid s(e)=s(f), r(e)=r(f)\right\},
$$

and $\mathscr{G}_{N}$ is $X \times G L(N, \mathbb{C}) \times X$. The map $i_{E} \times i_{\bar{E}}$ is an embedding and $p_{1}$ is the projection onto the first factor. Below we shall use the same letters for the corresponding embeddings and projections of $\tilde{P}_{E}, \tilde{P}_{\bar{E}}$ and $\tilde{P}_{\underline{N}}$. Observe that when we identify $\mathscr{G}_{\underline{N}}$ with $X \times G L(N) \times X$ and $\tilde{P}_{\underline{N}}$ with $X \times G L(N)$, then $\mathscr{G}_{\underline{N}} \times_{X \times X} \mathscr{G}_{\underline{N}}$ becomes isomorphic to $X \times G L(N) \times G L(N) \times X$, corresponding map $\bar{M}$ will be given by the product of matrices:

$$
M(x, g, h, y)=(x, g h) .
$$

Let us choose a connection in $\underline{N}$, which preserves the subbundles $E$ and $\bar{E}$. For example, one can take $A=p d p-q d q$, the Grassmanian connection, generated by $p$ (see [11]). Then $\Psi^{*}$ will be equal to the following composition:

$$
\begin{aligned}
& \Omega_{D R}\left(\tilde{P}_{E}\right) \stackrel{p_{1}^{*}}{\longrightarrow} \Omega_{D R}\left(\tilde{P}_{E} \times{ }_{X} \tilde{P}_{\bar{E}}\right) \\
& \quad \stackrel{M^{*}}{\longrightarrow} \Omega_{D R}\left(\mathscr{G}_{E} \times X \times X\right) \mathscr{G}_{\bar{E}} \otimes_{\Omega_{D R}(X)^{e v}} \Omega_{D R}\left(\mathscr{G}_{E} \times X \times X\right) \mathscr{G}_{\bar{E}} \\
& \quad \stackrel{\int_{\gamma} A}{\longrightarrow} \Omega_{D R}\left(\mathscr{G}_{E} \times X \times X\right) \mathscr{G}_{\bar{E}} \otimes_{\Omega_{D R}(X)^{e v}} \Omega_{D R}(P(X))
\end{aligned}
$$

Here we used a quasi-isomorphism between $\Omega_{D R}\left(\left(\mathscr{G}_{E} \times X \times X\right) \mathscr{G}_{\bar{E}} \times X_{X^{o p}}\right.$ $\left(\mathscr{G}_{E} \times_{X \times X} \mathscr{G}_{\bar{E}}\right)$ and $\Omega_{D R}\left(\mathscr{G}_{E} \times_{X \times X}\right) \mathscr{G}_{\bar{E}} \otimes_{\Omega_{D R}(X)^{e v}} \Omega_{D R}\left(\mathscr{G}_{E} \times X \times X\right) \mathscr{G}_{\bar{E}} ;$ it can be realized as follows: for a form $\omega$ in $\Omega_{D R}\left(\mathscr{G}_{E} \times X \times X\right) \mathscr{G}_{\bar{E}}$ consider an element $\tilde{\omega}$ in the differential forms on $\mathscr{G}_{\underline{N}}$, which maps into $\omega$ under $\left(i_{1} \times i_{2}\right)^{*}$ (see the diagram (4.5)), apply $\int_{\gamma} A \overline{\text { to }} \tilde{\omega}$ and observe that the result doesn't depend on the choice of $\tilde{\omega}$. Finally to complete the construction we should observe, that the differential forms in $\Omega_{D R}\left(\mathscr{G}_{E} \times_{X \times X}\right) \mathscr{G}_{\bar{E}}$ that 
we obtain by this sequence of homomorphisms are basic with respect to the projection $p_{1}$, so they belong to $\Omega_{D R}\left(\mathscr{G}_{E}\right)$.

Now, if we use the Chen's iterated integral map, we obtain the following description of $\Psi^{*}$ : for any form $\omega$, polynomial on the fibres of $\tilde{P}_{E}$, we choose a representative $\omega^{\prime} \otimes \omega^{\prime \prime} \in \Omega_{D R}(X) \otimes \Omega_{\text {poly }}^{*}(G L(N))=\Omega_{\text {vpoly }}^{*}\left(\tilde{P}_{\underline{N}}\right)$. Then $M^{*}\left(\omega^{\prime} \otimes \omega^{\prime \prime}\right)=\omega^{\prime} \otimes\left(\omega^{\prime \prime}(1) \otimes \omega^{\prime \prime}(2)\right) \otimes 1 ;$ we apply the homological monodromy map to $\omega^{\prime \prime}(2)$, thus obtaining an element in $\Omega_{D R}\left(\mathscr{G}_{\underline{N}}\right) \otimes B\left(\Omega_{D R}(X)\right)$. Finally, we apply $\left(i_{1} \times i_{2}\right)^{*}$ to the first tensor factor and observe that the result doesn't depend on coordinates in the $\bar{E}$ direction.

An important consequence of these considerations is the following

LEMMA 4.4. - Let $A$ be a grassmanian connection in $\underline{N}$, associated with the projector $p$, defining $E$, then the map $\tilde{\phi}_{E}$ is equal to the following composition:

$$
\begin{aligned}
\Omega_{D R}\left(\tilde{P}_{E}\right) & \stackrel{\sigma^{-1} \Psi^{*}}{\longrightarrow} \Omega_{D R}\left(\mathscr{G}_{E}\right) \otimes_{\Omega_{D R}(X)^{e v}} \bar{B}\left(\Omega_{D R}(X)\right) \\
& \stackrel{I^{*} \otimes m}{\longrightarrow} \Omega_{D R}\left(\tilde{P}_{E}\right) \otimes_{\Omega_{D R}(X)} N H\left(\Omega_{D R}(X)\right) \stackrel{1^{*} \otimes I d}{\longrightarrow} N H\left(\Omega_{D R}(X)\right),
\end{aligned}
$$

where $I: \tilde{P}_{E} \rightarrow \mathscr{G}_{E}$ is a natural inclusion, $m: B\left(\Omega_{D R}(X)\right) \rightarrow N H\left(\Omega_{D R}(X)\right)$ is the map, given by multiplication of the the leftmost and the rightmost tensors and normalization, and $1: X \rightarrow \tilde{P}_{E}$ is the unit section.

Proof. - The proof of this fact follows directly from the construction of map $\Psi^{*}$, see (4.6).

Let us now obtain a similar description of the map $\tilde{\hat{\phi}}$, determined by a twisting cochain $\phi$. To this end we first of all observe that the map $I^{*}$ is homotopy equivalent to $\tilde{I}: G P\left(\Omega_{D R}(X), \Omega_{G}\right) \rightarrow \Omega_{G} \hat{\otimes}_{\phi} \Omega_{D R}(X)$ from the diagram (4.4), and the map $1^{*}$ is equal to $\tilde{\epsilon}: \Omega_{G} \hat{\otimes}_{\phi} \Omega_{D R}(X) \rightarrow \Omega_{D R}(X)$, where $\epsilon$ is counit in $\Omega_{G}, \epsilon$ extends to $\Omega_{G} \hat{\otimes}_{\phi} \Omega_{D R}(X)$ because $\Omega_{D R}(X)$ is commutative. It is now our purpose to find a map

$$
\tilde{\Psi}^{*}: \Omega_{G} \hat{\otimes}_{\phi} \Omega_{D R}(X) \rightarrow G P\left(\Omega_{D R}(X), \Omega_{G}\right) \otimes_{\Omega_{D R}(X)^{e v}} \tilde{B}\left(\Omega_{D R}(X)\right),
$$

such that $\tilde{\hat{\phi}}=(\tilde{\epsilon} \otimes I d)(\tilde{I} \otimes m) \tilde{\Psi}^{*}$, (we denote $\tilde{B}\left(\Omega_{D R}(X)\right)=\bar{B}\left(\Omega_{D R}(X)\right.$, $\left.\left.\Omega_{D R}(X), \Omega_{D R}(X)\right)\right)$. In order to find $\tilde{\Psi}^{*}$, observe, that the complex $G P\left(\Omega_{D R}(X), \Omega_{G}\right) \otimes_{\Omega_{D R}(X) \text { ev }} B\left(\Omega_{D R}(X)\right)$ is quasi-isomorphic to $\Omega_{G} \hat{\otimes}_{\phi}$ $\Omega_{D R}(X)$, the quasi-isomorphism being given by

$$
\begin{gathered}
\tilde{\Phi}^{*}=I d \otimes_{\Omega_{D R}(X)^{e v}} \operatorname{Proj}_{0}: G P\left(\Omega_{D R}(X), \Omega_{G}\right) \otimes_{\Omega_{D R}(X)^{e v}} \tilde{B}\left(\Omega_{D R}(X)\right) \\
\rightarrow \Omega_{G} \hat{\otimes}_{\phi} \Omega_{D R}(X) \\
-356-
\end{gathered}
$$


where

$$
\operatorname{Proj}_{0}\left(a\left[a_{1}|\ldots| a_{n}\right] b\right)=\left\{\begin{array}{cc}
0, & n \geqslant 1 \\
a b, & n=0 .
\end{array}\right.
$$

To prove that $\tilde{\Phi}^{*}$ is quasi-isomorphism, one can use the standard spectral sequence technique. Here we shall describe an explicit homotopy inverse map for $\tilde{\Phi}^{*}$; it is this inverse map, that shall play the rôle of $\tilde{\Psi}^{*}$. We put:

$$
\tilde{\Psi}^{*}(k \otimes a)=k \otimes(1[] a)+\sum_{n=1}^{\infty} k^{(1)} \otimes\left(1\left[\phi\left(k^{(2)}\right)|\ldots| \phi\left(k^{(n+1)}\right)\right] a\right),
$$

where $k \otimes a$ is an arbitrary element in $\Omega_{G} \hat{\otimes}_{\phi} \Omega_{D R}(X), k \in \Omega_{G}, a \in \Omega_{D R}(X)$, and the tensor product on the right hand side is taken over $\Omega_{D R}(X)^{e v}$ (we omit the left and right $\Omega_{D R}(X)$ tensors in $\left.G P\left(\Omega_{D R}(X), \Omega_{G}\right)\right)$. It is easy to show that $\tilde{\Psi}^{*}$, given by this formula, commutes with the differential. Indeed, it is only necessary to check that it commutes with the "left" part of the twisting, i.e.:

$$
\begin{aligned}
\tilde{\Psi}^{*}\left(d k \otimes a+(-1)^{|k|} k \otimes d a+(-1)^{\left|k^{(1)}\right|} k^{(1)} \otimes \phi\left(k^{(2)}\right) a\right) \\
=(d \otimes I d+I d \otimes b+1 \cap \phi) \tilde{\Psi}^{*}(k \otimes a),
\end{aligned}
$$

where $b$ is the total differential in $\tilde{B}\left(\Omega_{D R}(X)\right)$ and

$$
1 \cap \phi\left(k \otimes\left(a 1\left[a_{2}|\ldots| a_{n}\right] a_{n+1}\right)\right)=(-1)^{\left|k^{(1)}\right|} k^{(1)} \otimes\left(\phi\left(k^{(2)}\right) a_{1}\left[a_{2}|\ldots| a_{n}\right] a_{n+1}\right) .
$$

We compute both expressions. On the left hand side we have (here are only the first three terms from the formula (4.7), the argument of the left hand side of $(4.8)$ is denoted $L(k \otimes a))$ :

$$
\begin{aligned}
\tilde{\Psi}^{*}( & L(k \otimes a))=d k \otimes(1[] a)+(-1)^{|k|} k \otimes(1[] d a) \\
& +(-1)^{\left|k^{(1)}\right|} k^{(1)} \otimes\left(1[] \phi\left(k^{(2)}\right) a\right) \\
& +d k^{(1)} \otimes\left(1\left[\phi\left(k^{(2)}\right)\right] a\right)+(-1)^{\left|k^{(1)}\right|} k^{(1)} \otimes\left(1\left[\phi\left(d k^{(2)}\right)\right] a\right) \\
& +(-1)^{|k|} k^{(1)} \otimes\left(1\left[\phi\left(k^{(2)}\right)\right] d a\right)+(-1)^{\left|k^{(1)}\right|} k^{(1)} \otimes\left(1\left[\phi\left(k^{(2)}\right)\right] \phi\left(k^{(3)}\right) a\right) \\
& +d k^{(1)} \otimes\left(1\left[\phi\left(k^{(2)}\right) \mid \phi\left(k^{(3)}\right)\right] a\right)+(-1)^{\left|k^{(1)}\right|} k^{(1)} \otimes\left(1\left[\phi\left(d k^{(2)}\right) \mid \phi\left(k^{(3)}\right)\right] a\right) \\
& +(-1)^{\left|k^{(1)}\right|+\left|k^{(2)}\right|} k^{(1)} \otimes\left(1\left[\phi\left(k^{(2)}\right) \mid \phi\left(d k^{(3)}\right)\right] a\right) \\
& +(-1)^{|k|} k^{(1)} \otimes\left(1\left[\phi\left(k^{(2)}\right) \mid \phi\left(k^{(3)}\right)\right] d a\right) \\
& +(-1)^{\left|k^{(1)}\right|} k^{(1)} \otimes\left(1\left[\phi\left(k^{(2)}\right) \mid \phi\left(k^{(3)}\right)\right] \phi\left(k^{(4)}\right) a\right)+\ldots
\end{aligned}
$$

On the other hand,

$\tilde{\Psi}^{*}(k \otimes a)=k \otimes(1[] a)+k^{(1)} \otimes\left(1\left[\phi\left(k^{(2)}\right)\right] a\right)+k^{(1)} \otimes\left(1\left[\phi\left(k^{(2)}\right) \mid \phi\left(k^{(3)}\right)\right] a\right)+\ldots$. 
If we apply $(d \otimes I d+I d \otimes b+1 \cap \phi)$ to the first two terms, we obtain:

$$
\begin{aligned}
d k \otimes & (1[] a)+(-1)^{|k|} k \otimes(1[] d a)+(-1)^{\left|k^{(1)}\right|} k^{(1)} \otimes\left(\phi\left(k^{(2)}\right)[] a\right) \\
& +d k^{(1)} \otimes\left(1\left[\phi\left(k^{(2)}\right)\right] a\right)+(-1)^{\left|k^{(1)}\right|} k^{(1)} \otimes\left(1\left[d \phi\left(k^{(2)}\right)\right] a\right) \\
& +(-1)^{|k|} k^{(1)} \otimes\left(1\left[\phi\left(k^{(2)}\right)\right] d a\right)+(-1)^{\left|k^{(1)}\right|+1} k^{(1)} \otimes\left(\phi\left(k^{(2)}\right)[] a\right) \\
& +(-1)^{\left|k^{(1)}\right|} k^{(1)} \otimes\left(1[] \phi\left(k^{(2)}\right) a\right)+(-1)^{\left|k^{(1)}\right|} k^{(1)} \otimes\left(\phi\left(k^{(2)}\right)\left[\phi\left(k^{(3)}\right)\right] a\right)
\end{aligned}
$$

The first, the second, the fourth, the sixth and the seventh terms in this formula coincide with the first, the second, the fourth, the sixth and the third terms in (4.9) respectively, the third and the sixth terms of this formula cancel each other, and the fifth is equal to $(-1)^{\mid k^{(1)}} \mid\left(k^{(1)} \otimes\left(1\left[\phi\left(d k^{(2)}\right)\right] a\right)+\right.$ $(-1)^{\left|k^{(1)}\right|+1}\left(k^{(1)} \otimes\left(1\left[\phi\left(k^{(2)}\right) \phi\left(k^{(3)}\right)\right] a\right)\right.$, since $d \phi+\phi \cup \phi=0$. The first term in this expression cancels with the fifth term in (4.9), and the second one will cancel with a term from $(I d \otimes b)\left(k^{(1)} \otimes\left(1\left[\phi\left(k^{(2)}\right) \mid \phi\left(k^{(3)}\right)\right] a\right)\right)$. Further calculations by induction will give the same results.

Thus, $\tilde{\Psi}^{*}$ is a chain map. One can find a chain homotopy $\tilde{\Upsilon}$, such that $\tilde{\Psi}^{*} \tilde{\Phi}^{*}-I d=d \tilde{\Upsilon}-\tilde{\Upsilon} d$, but this is not quite necessary, since we know, that $\tilde{\Phi}^{*}$ induces an isomorphism in homology, and on the other hand, it is evident that $\tilde{\Phi}^{*} \tilde{\Psi}^{*}=I d$. In fact, one can obtain $\tilde{\Psi}^{*}$ and the corresponding chain homotopy with the help of the perturbation Lemma. To this end, consider a "smaller" differential on $G P\left(\Omega_{D R}(X), \Omega_{G}\right) \otimes_{\Omega_{D R}(X) \text { ev }} \tilde{B}\left(\Omega_{D R}(X)\right)$ given by the sum of the usual differential in the tensor product and the additional part, given by the twisting cochain. There exists an evident homotopy equivalence between $\Omega_{G} \otimes \Omega_{D R}(X)$ and $G P\left(\Omega_{D R}(X), \Omega_{G}\right) \otimes_{\Omega_{D R}(X)^{e v}} \tilde{B}\left(\Omega_{D R}(X)\right)$ if we throw away the twisting cochain from the differential on the left hand side too. The additional terms, containing $\phi$ can be regarded as a small perturbation of the differential and the formulas, similar to $(2.21)$, give the homotopy inverse $\tilde{\Psi}^{*}$ and $\tilde{\Upsilon}$.

It is now evident that $\tilde{\hat{\phi}}=(\tilde{\epsilon} \otimes I d)(\tilde{I} \otimes m) \tilde{\Psi}^{*}$. Thus the theorem follows from the next homotopy-commutative diagram (see next page).

Here $\mathscr{I} \mathscr{J}$ are the homotopy equivalences from (4.4), and $\mathscr{J}$ is also the homotopy equivalence from the proposition 3.3. We have the following sequence of equalities up to homotopy:

$$
\begin{aligned}
\tilde{\phi}_{E} \mathscr{\mathscr { J }}=\left(1^{*} \otimes I d\right)\left(I^{*} \otimes m\right) \sigma^{-1} \Psi^{*} \mathscr{J} & =\left(1^{*} \otimes I d\right)\left(I^{*} \otimes m\right)(I \otimes I d) \tilde{\Psi}^{*} \\
& =(\tilde{\epsilon} \otimes I d)(\tilde{I} \otimes m) \tilde{\Psi}^{*}=\tilde{\hat{\phi}} .
\end{aligned}
$$

In fact we have showed, that $\tilde{\phi}_{E}$ and $\tilde{\hat{\phi}}$ are homotopic as homomorphisms of coalgebras over $\Omega_{D R}(X)$. Indeed, all the maps, involved here commute (up to a homotopy) with the coproducts. 


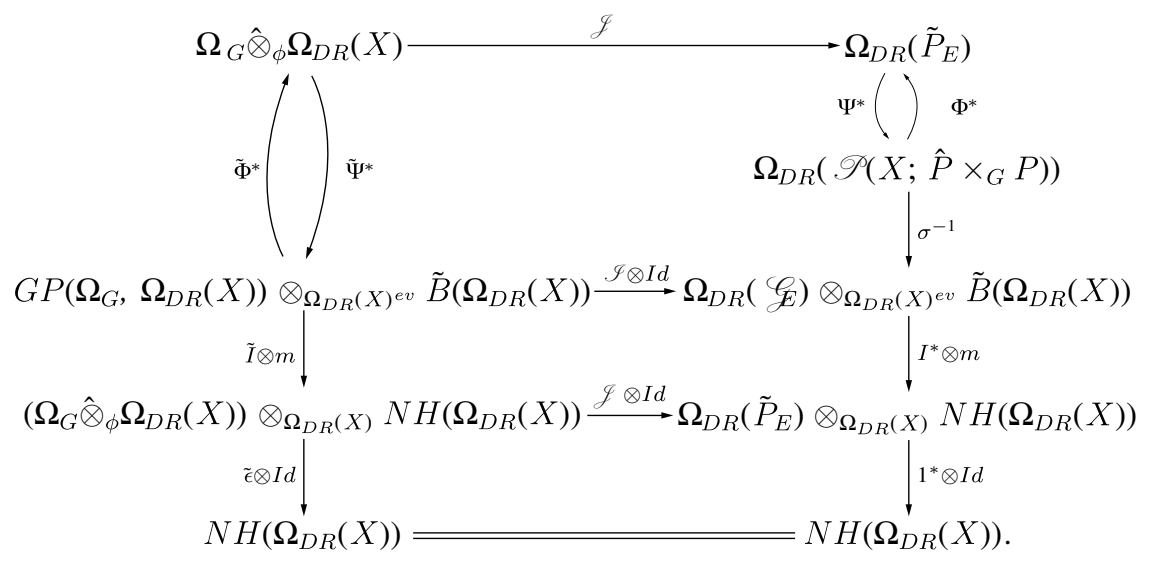

\subsection{Equivariantization and Bismut classes}

In this section we endeavor to explain in what way one can reproduce the cyclic Chern character of Bismut $\operatorname{ch}(\nabla)$ (or, rather its Getzler-JonesPetrack version $\operatorname{ch}(p, \mathscr{A}))$ in the framework of our theory. Recall that this class belongs to the $\mathbb{C}\left[\left[u^{-1}, u\right]\right.$-linear cyclic cohomology of the equivariant complex $\Omega_{D R}(X \times \mathbb{T})^{\mathbb{T}}\left[\left[u^{-1}, u\right]\right.$, where $\mathbb{T}=S^{1}$. The latter algebra is the $\mathbb{T}$-equivariant complex of the cartesian product $X \times \mathbb{T}$. In effect, usual $\mathbb{T}$ equivariant complex should be an algebra over the polynomial ring $\mathbb{C}[u]$, but in our case we consider the tensor product over $\mathbb{C}[u]$ of this complex with the formal Laurent series ring. The condition of $\mathbb{C}\left[\left[u^{-1}, u\right]\right.$-linearity means that one should consider all the tensor products over this ring. We shall give a description of the cyclic Chern character in terms of the GJP map (see previous section) and discuss an analogous construction that involves twisting cochains. For any twisting cochain $\phi$ with domain $K$ these constructions will give a series of cocycles in normalized cyclic complex of the algebra $A_{\mathbb{T}}$, where $A$ is the range of $\phi$ and $A_{\mathbb{T}}$ is the equivariantized version of $A$, see definition below. Similarly to the paper [11], one can prove that the homology of the normalized cyclic complex is equal to the equivariant cohomology of the free loop space. However, these classes depend on the choice of $\phi$, and in particular on $K$ and $A$. As for dependence on $\phi$, one can hope to get rid of it in the manner similar to Zamboni's, [27], proof of the independence of Getzler-Jones-Petrack's class of the choice of projector $p$; this question deserves a separate paper. It would be nice to have a more invariant description of these classes, e.g. a description, independent 
of the choice of $K$. But this should be done in the more general context of homotopy theory, which we postpone for future.

Let us first of all recall the definition of Getzler-Jones-Petrack's class $\operatorname{ch}(p, \mathscr{A})$ : it is the 0-degree class in cyclic homology of the algebra $\Omega_{D R}(X \times \mathbb{T})^{\mathbb{T}}\left[\left[u^{-1}, u\right]\right.$ (see below) determined by the closed chain:

$$
\operatorname{ch}(p, \mathscr{A})=\sum_{n} \operatorname{Tr}(p, \mathscr{A}, \ldots, \mathscr{A})
$$

where for an $n+1$-tuple of $N \times N$ matrices $A^{0}, A^{1}, \ldots, A^{n}$ we put

$$
\operatorname{Tr}\left(A^{0}, A^{1}, \ldots, A^{n}\right)=\sum_{i_{0}, \ldots, i_{n}=1}^{N} A_{i_{0} i_{1}}^{0} \otimes A_{i_{1} i_{2}}^{1} \otimes \ldots \otimes A_{i_{n} i_{0}}^{n} .
$$

In our case we take $p$ to be the projector-valued function on $X$, which determines $E$, and $\mathscr{A}$ a flat $\mathbb{T}$-equivariant connection on $X \times \mathbb{T}$, given by the formula:

$$
\mathscr{A}=A+\frac{F d t}{u} .
$$

Here $A=p d p$ is the connection on $E \subseteq \underline{N}$, determined by $p$, and $F=$ $p d p d p$ is its curvature. Both $A$ and $F$ should be regarded as matrix-valued differential forms on $X$. One can check, that the following equations hold (see [11], §6)

$$
\begin{aligned}
d_{u} p+[\mathscr{A}, p] & =0, \\
d_{u} \mathscr{A}+\frac{1}{2}[\mathscr{A}, \mathscr{A}] & =0 .
\end{aligned}
$$

The differential $d_{u}$ is the $\mathbb{T}^{1}$-equivariant differential on $\Omega_{D R}(X \times \mathbb{T})^{\mathbb{T}} \otimes_{\mathbb{C}[u]}$ $\left.\left.\mathbb{C}\left[u, u^{-1}\right]\right] \cong \Omega_{D R}(X)[1, d t] \otimes \mathbb{C}\left[u, u^{-1}\right]\right]$ (this algebra is denoted by $\Omega_{\mathbb{T}}(X \times$ $\mathbb{T})$ in the cited paper; for the sake of brevity we shall stick to the same notation below):

$$
\begin{gathered}
d_{u}\left(\operatorname{ap}\left(u, u^{-1}\right)\right)=\operatorname{dap}\left(u, u^{-1}\right), \\
d_{u}\left(b d t q\left(u, u^{-1}\right)\right)=\operatorname{dbdt} q\left(u, u^{-1}\right)+(-1)^{|b|} b u q\left(u, u^{-1}\right) .
\end{gathered}
$$

It follows from these equations that $\operatorname{ch}(p, \mathscr{A})$ is a cocycle in the normalized cyclic complex $\tilde{N}\left(\Omega_{D R}^{X}\right)$, where we once again use the notation of $\S 6$ of the article [11]:

$$
\tilde{N}\left(\Omega_{D R}^{X}\right)=\tilde{C}\left(\Omega_{D R}(X)\right) / \tilde{D}\left(\Omega_{D R}(X)\right)
$$

where

$$
\begin{gathered}
\tilde{C}\left(\Omega_{D R}(X)\right)=C_{\Omega_{\mathbb{T}}}\left(\Omega_{\mathbb{T}}(X \times \mathbb{T})\right) \widehat{\otimes}_{\Omega_{\mathbb{T}}} \mathbb{C}\left[\left[u^{-1}, u\right]\right. \\
-360-
\end{gathered}
$$


is the cyclic complex over $\Omega_{\mathbb{T}}=\mathbb{C}[u]$. Note that it doesn't matter, whether we take tensor products of $\Omega_{\mathbb{T}}(X \times \mathbb{T})$ over $\mathbb{C}[u]$ or of $\Omega_{\mathbb{T}}(X \times \mathbb{T}) \otimes_{\mathbb{C}[u]}$ $\mathbb{C}\left[\left[u^{-1}, u\right]\right.$ over $\mathbb{C}\left[\left[u^{-1}, u\right]\right.$. And $\tilde{D}\left(\Omega_{D R}(X)\right)$ is the subcomplex generated by cyclic operations applied to degenerate cochains in $\tilde{C}\left(\Omega_{D R}(X)\right)$, i.e. to cochains, containing an element of $C^{\infty}(X)$ in one of their tensor legs, except for the rightmost. Our purpose now is to obtain $\operatorname{ch}(p, \mathscr{A})$ as the image of an element under the map, similar to $\tilde{\phi}_{E}$. To this end we need to modify $\tilde{\phi}_{E}$ so that its domain and range become $\mathbb{T}$-equivariant complexes and then pass to the cyclic complex on the right. Take notice that in our case we shall write the non-normalized tensor leg in the definition of $\tilde{N}\left(\Omega_{D R}^{X}\right)$ in the right of the tensor product, rather than in the left, as it is done in the paper [11] (the reader should also refer to this paper for further details on cyclic complexes and cyclic homology).

So let $A=p d p, F=p d p d p$ be the grassmanian connection on $E$ and its curvature. We regard them as matrix-valued differential forms on $X$. Recall (see section 4.1), that $\tilde{\phi}_{E}$ is generated by the derivative $\phi_{E}=\sum_{i, j}\left(A^{i j} \otimes\right.$ $X_{i j}+F^{i j} \otimes I_{i j}$ ) (it acts on $\Omega_{D R}(X) \otimes \Omega_{D R}(G L(N))$ and preserves the ideal, which determines $\left.\tilde{P}_{E}\right)$. One can extend $\phi_{E}$ to a $\left.\mathbb{C}\left[d t, u, u^{-1}\right]\right]$-linear derivative on the equivariant complex $\Omega_{\mathbb{T}}\left(\tilde{P}_{E} \times \mathbb{T}\right)$ : just put $\phi_{E}(u)=\phi_{E}(d t)=0$. It is easy to check that this extension preserves the usual equations of the twisting maps ( $\phi_{E}$ is a twisting map as a derivative on $\Omega_{D R}(G L(N))$ ), i.e.

$$
\left[\phi_{E}^{u}, d_{u}\right]+\left(1 \otimes \phi_{E}^{u}\right) \phi_{E}^{u}=0 .
$$

This means that we can use the usual formula for $\tilde{\phi}_{E}$ to obtain a map $\tilde{\phi}_{E}^{u}: \Omega_{\mathbb{T}}\left(\tilde{P}_{E} \times \mathbb{T}\right) \rightarrow N H_{\Omega_{\mathbb{T}}}\left(\Omega_{\mathbb{T}}(X \times \mathbb{T})\right.$ ) (the subscript $\Omega_{\mathbb{T}}$ on the right means that we consider the $\mathbb{C}[u]$-linear version of the Hochschild complex). We put:

$$
\tilde{\phi}_{E}^{u}=\left.\sum_{n}\left(\phi_{E}^{u}\right)^{\otimes n}\right|_{1}
$$

(1 is the unit of $G L(N)$ ). On the other hand, one can consider another twisting map on $\Omega_{D R}(G L(N)$ ) with values in the equivariant complex of $X \times \mathbb{T}$ and use it to induce a map to $\Omega_{\mathbb{T}}\left(\tilde{P}_{E} \times \mathbb{T}\right)$ : put

$$
\psi_{E}^{u}=\sum_{i, j}\left(A_{i j} \otimes e_{i j}-u^{-1} F_{i j} d t \otimes e_{i j}\right) .
$$

Since the equivariant curvature of the equivariant connection $\mathscr{A}$ vanishes, $\psi_{E}^{u}$ is a twisting map. Now we can restrict it to the factor-algebra $\Omega_{D R}\left(\tilde{P}_{E}\right)$ of $\Omega_{D R}(X) \otimes \Omega_{D R}(G L(N))$ and pass to the equivariant complex $\Omega_{\mathbb{T}}\left(\tilde{P}_{E} \times \mathbb{T}\right)$. Finally, we put:

$$
\begin{aligned}
\tilde{\psi}_{E}^{u}= & \left.\sum_{n}\left(\psi_{E}^{u}\right)^{\otimes n}\right|_{1} . \\
& -361-
\end{aligned}
$$


The following proposition is an important step towards the fuller understanding of Getzler-Jones-Petrack class.

\section{Proposition 4.5. -}

(i) Twisting maps $\phi_{E}^{u}$ and $\psi_{E}^{u}$ are gauge equivalent (notion of gauge equivalence of twisting maps is similar to that of twisting cochains, see section 1.3 and discussion below);

(ii) The representative of $c h(p, \mathscr{A})$ in the Hochschild complex is equal to $\tilde{\psi}_{E}^{u}\left(\operatorname{tr}_{u}\right)$, where $\operatorname{tr}_{u}$ is certain equivariantly-closed element in $\Omega_{\mathbb{T}}\left(\tilde{P}_{E} \times\right.$ $\mathbb{T})$.

Proof. - First of all we shall give an explicit formula of the gauge transformation, relating $\phi_{E}^{u}$ and $\psi_{E}^{u}$. Put

$$
C=\sum_{i, j} u^{-1} F^{i j} d t \otimes \iota_{i j} .
$$

This is a degree 0 differentiation of $\Omega_{D R}(G L(N))$ with values in $\Omega_{\mathbb{T}}(X \times \mathbb{T})$; we can extend it to a differentiation on $\Omega_{\mathbb{T}}\left(\tilde{P}_{E} \times \mathbb{T}\right)$ with values in the same algebra. An easy computation shows that the following equation holds for $\phi_{E}^{u}, \psi_{E}^{u}$ and $C$ :

$$
\phi_{E}^{u}-\left[\phi_{E}^{u}, C\right]-d C=\psi_{E}^{u}
$$

and hence if we put $c=\exp (C)=1+C$, then $c^{-1}=1-C$ and $\psi_{E}^{u}=$ $c^{-1} \phi_{E}^{u} c+c^{-1} d c$.

Next, consider the function $\operatorname{Tr}_{n}$ on $G L(n)=G L(n ; \mathbb{C}), \operatorname{Tr}_{n}=\sum u_{i i}$. This function is $A d$-invariant, so we can extend it from a fibre of $\widetilde{P}_{E}$, to the whole $\tilde{P}_{E}$. Indeed, this is possible since one passes from one chart of $\tilde{P}_{E}$ to another by means of the adjoint action of the group on itself. We shall denote this extension by $t r_{E}$. This function is equal to the restriction of the function $\operatorname{Tr}_{N}$, or rather of $1 \otimes T r$, on $X \times G L(N)$ to $\tilde{P}_{E} \subseteq X \times G L(N)$, when we include $E$ into trivial bundle $X \times \mathbb{C}^{N}$; so $\operatorname{tr}_{E} \equiv 1 \otimes \operatorname{Tr}_{N}(\bmod (I)$, where $I$ is the ideal in $C^{\infty}(X) \otimes \mathcal{A}(G L(N))$, generated by $1_{N}-p(x)$. Similarly, one can extend $d T r_{n}$, to a degree 1 differential form $d t r_{E}$ on $\tilde{P}_{E}$, so that $d\left(t r_{E}\right)=d t r_{E}$, where on the left stands the usual de Rham differential on $\tilde{P}_{E}$; this form is equal to the restriction of $d \operatorname{Tr}_{N}$ onto $\tilde{P}_{E}$. Put $t r_{u}=$ $t r_{E}+u^{-1} d t r_{E} d t$. Clearly $t r_{u}$ is an equivariantly-closed form, $d_{u}\left(t r_{u}\right)=0$. In effect, $t r_{u}$ is equivariantly-exact: $t r_{u}=d_{u}\left(u^{-1} t r_{E} d t\right)$. Now it is a matter of a simple calculation to see that $\tilde{\psi}_{E}^{u}\left(t r_{u}\right)=\operatorname{ch}(p, \mathscr{A})$. The only thing one should use is that the Lie algebra of $G L(n)$ is spanned by the left-invariant 
vector fields $T_{i j}(g)=\sum_{k} u_{k i}(g) \frac{\partial}{\partial u_{k j}}$, and hence

$$
\begin{aligned}
\sum_{i, j} A_{i j} \otimes T_{i j}\left(u_{m n}\right) & =\sum_{i, j, k} A_{i j} \otimes u_{k i} \frac{\partial}{\partial u_{k j}}\left(u_{m n}\right)=\sum_{i, j, k} A_{i j} \otimes u_{k i} \delta_{k m} \delta_{n j} \\
& =\sum_{i} A_{i n} \otimes u_{m i}
\end{aligned}
$$

and that Lie derivatives commute with the de Rham differential and the restriction of $d t r_{E}$ on the unit section is equal to 0 .

In fact, the element $\operatorname{ch}(p, \mathscr{A})$ is exact as the element in the Hochschild complex. Namely, $\operatorname{ch}(p, \mathscr{A})=b\left(\tilde{\psi}_{E}^{u}\left(u^{-1} \operatorname{tr}_{E} d t\right)\right)$, but it is not exact in cyclic complex: $B\left(\tilde{\psi}_{E}^{u}\left(u^{-1} \operatorname{tr}_{E} d t\right)\right)$ is not a degenerate element, so $(b+u B)\left(\tilde{\psi}_{E}^{u}\left(u^{-1} t r_{E} d t\right)\right) \neq \operatorname{ch}(p, \mathscr{A})$ in $\tilde{N}\left(\Omega_{D R}^{X}\right)$.

It would be tempting to assume that $\phi_{E}^{u}\left(t r_{u}\right)$ gives the same cyclic class. Then we could perform the same equivariantization procedure with the map $\hat{\hat{\phi}}$ associated with twisting cochain $\phi$ and obtain the same class, applying $\tilde{\hat{\phi}}_{u}$ to the $t r_{n} \otimes 1+d t r_{n} \otimes u^{-1} d t \in \Omega_{D R}(G L(n)) \hat{\otimes}_{\phi} \Omega_{\mathbb{T}}(X \times \mathbb{T})$, which is corresponds to $t r_{u}$ under the equivalences of section 4.2. Unfortunately, element $\tilde{\phi}_{E}^{u}\left(t r_{u}\right)$ is not even closed with respect to the cyclic differential $B$ - its first tensor leg is proportional to $u^{-1} d t$. So one should change the construction of $\tilde{\hat{\phi}}_{u}$ a little bit, using the following idea. Let $\phi: K \rightarrow A$ be a twisting cochain, where $K$ is a DG coalgebra and $A$ a DG algebra. Consider the formal equivariantization of $\left.A: A_{\mathbb{T}}=A \otimes\left(\mathbb{C}[1, d t] \otimes \mathbb{C}\left[u, u^{-1}\right]\right]\right)$ with a $\Omega_{T}$-linear differential $d_{u}$ :

$$
d_{u}\left(a^{\prime}+a^{\prime \prime} d t\right)=d a^{\prime}+d a^{\prime \prime} d t+(-1)^{\left|a^{\prime \prime}\right|} u a^{\prime \prime} .
$$

We can consider the $\phi$-twisted tensor product of $K$ and $A_{\mathbb{T}}$. Let $K_{o}$ denote the coalgebra $K$ regarded as coalgebra with trivial differential. Then the following proposition holds:

Proposition 4.6. - The twisted tensor product $K \otimes_{\phi} A_{\mathbb{T}}$ is isomorphic to the chain complex: $K_{o} \otimes_{\phi_{o}} A_{\mathbb{T}}$, where $\phi_{o}$ is the twisting cochain $\phi_{o}: K_{o} \rightarrow$ $A_{\mathbb{T}}$, given by the formula:

$$
\phi_{o}(k)=\phi(k)+u^{-1} \phi(d k) d t .
$$

Proof. - Consider the map $O: K \otimes A_{\mathbb{T}} \rightarrow K \otimes A_{\mathbb{T}}$ :

$$
O(k \otimes a)=k \otimes a-d k \otimes u^{-1} a d t .
$$


This map is invertible, its inverse is given by

$$
O^{-1}(k \otimes a)=k \otimes a+d k \otimes u^{-1} a d t .
$$

Using $O$, we construct the deformed differential $O^{-1} \circ d_{\phi} \circ O$. An easy calculation shows that the latter differential is equal to $(-1)^{|k|}\left(k \otimes d a+k^{(1)} \otimes\right.$ $\left.\phi\left(k^{(2)}\right) a+k^{(1)} \otimes u^{-1} \phi\left(k^{(2)}\right) d t a\right)=d_{\phi_{o}}(k \otimes a)$ on an element $k \otimes a$.

Similarly, one can deform the differential in $K \hat{\otimes}_{\phi} A$ so that it becomes isomorphic to $K_{o} \hat{\otimes}_{\phi_{o}} A$; since the differential in $K_{o}$ vanishes, we conclude that an element $k \otimes 1 \in K_{o} \hat{\otimes}_{\phi_{o}} A$ is closed if and only if it verifies the equation

$$
k^{(1)} \otimes \phi_{o}\left(k^{(2)}\right)=k^{(2)} \otimes \phi_{o}\left(k^{(1)}\right) .
$$

In particular, this equation holds, if $k$ is in the cocenter of $K$, i.e. $k^{(1)} \otimes k^{(2)}=$ $k^{(2)} \otimes k^{(1)}$. So $k \otimes 1$ is closed in $K_{o} \hat{\otimes}_{\phi_{o}} A$ for all $k$ in cocenter. For instance, if $K=\Omega_{D R}(G L(n))$ one can take $k=\sum u_{i i}=t r_{n}$. So one obtains a closed element $\tilde{\hat{\phi}}_{o}\left(t r_{n} \otimes 1\right)$ in $\tilde{N}\left(A_{\mathbb{T}}\right)$, where the normalized cyclic complex $\tilde{N}\left(A_{\mathbb{T}}\right)$ is given by the same construction as $\tilde{N}\left(\Omega_{D R}^{X}\right)$ before, see [11]. Repeating the reasoning of $\S 5$ [11] one concludes that the cohomology of $\tilde{N}\left(A_{\mathbb{T}}\right)$ is equal to the equivariant cohomology of the free loop space of $X$ as soon as $A$ is quasiequivalent to $\Omega_{D R}(X)$. It is obvious, that $\tilde{\hat{\phi}}_{o}\left(t r_{n} \otimes 1\right)$ is closed in reduced complex with respect to the cyclic operator $B$ (its last tensor leg is equal to $1 \in A_{\mathbb{T}}$ ). We shall denote the element, determined by this construction by $\operatorname{ch}(\phi, k)$ (here $\phi$ is a twisting cochain and $k \in K$ is a cocentral element). In particular, we obtain the following class $c h\left(\phi_{P}, t r_{n}\right)$, where $\phi_{P}$ is the cochain, defined in section 2.1

$$
\begin{gathered}
\operatorname{ch}\left(\phi_{P}, t r_{n}\right)=\sum_{n=0}^{\infty} \sum_{i_{1}, \ldots, i_{n}}\left\{\delta^{i_{1} i_{2}}-g_{\alpha \beta}^{i_{1} i_{2}}+u^{-1} d g_{\alpha \beta}^{i_{1} i_{2}} d t\right\} \otimes \\
\left.\left\{\delta^{i_{2} i_{3}}-g_{\alpha \beta}^{i_{2} i_{3}}+u^{-1} d g_{\alpha \beta}^{i_{2} i_{3}} d t\right\} \otimes \ldots \otimes\left\{\delta^{i_{n} i_{1}}-g_{\alpha \beta}^{i_{n} i_{1}}+u^{-1} d g_{\alpha \beta}^{i_{n} i_{1}} d t\right\} \otimes 1.10\right)
\end{gathered}
$$

Here $A=\check{C}_{\mathcal{U}}^{*}\left(X, \Omega_{D R}(U)\right), g_{\alpha \beta}: U_{\alpha \beta} \rightarrow G L(n)$ is the cocycle, defining of the $P$ and $\delta^{i j}$ is the Kronecker symbol. This formula defines a class in the cyclic cohomology of $A_{\mathbb{T}}$, which is isomorphic to the cohomology of free loop space of $X$ if $X$ is 1-connected.

In general the class of an element $\operatorname{ch}(\phi, k)$ can depend on the choice of the twisting cochain $\phi$. Indeed, if $\phi^{\prime}$ is another cochain, equivalent to $\phi$ via a transformation map $c: K \rightarrow A$, then one can extend $c$ to an equivalence $c_{o}$ between the deformed cochains $\phi_{o}$ and $\phi_{o}^{\prime}$ : put $c_{o}(k)=c(k)+u^{-1} c(d k) d t$. The formula (3.36) gives a connecting homotopy that allows one compare 
the elements $\tilde{\hat{\phi}}_{o}(k \otimes 1)$ and $\tilde{\hat{\phi}}_{o}^{\prime}(k \otimes 1)$. As one can see $B\left(\hat{H}_{c_{o}}(k \otimes 1)\right)$ can be different from 0 , since it can contain such elements as $c(d k) d t$ at the last tensor leg. Thus the classes we obtain can depend on $\phi$. One can try to prove their independence on the choices made by a more delicate reasoning, similar to that of Zamboni [27]. On the other hand, if we chose the twisitng cochain $\xi$ from the section 2.3 , then we evidently obtain a class, that will be equal to Bismut's class after globalization (since they coincide on every local plot in $\mathcal{L} X$ in the sense of Chen).

\section{Bibliography}

[1] Anderson (D.W.). - A generalization of the Eilenberg-Moore spectral sequence, Bulletin of the Amer. Math. Soc. 78 Number 5, p. 784-786 (1972).

[2] Bismut (J.-M.). - Le théorème d'Atiyah-Singer pour les opérateurs elliptiques classiques: une approche probabiliste C. R. Acad. Sci. Paris Sér. I Math., 297 No.8, p. 481-484 (1983)

[3] Botт (R.), Tu (L.W.). - Differential Forms in Algebraic Topology, SpringerVerlag, Berlin-Heiderberg-New York, (1982).

[4] Bott (R.), Segal (G.). - The Cohomology of the Vector Fields on a Manifold, Topology, 16, p. 285-298 (1977).

[5] Bousfield (A. K.), Gugenheim (V. K. A. M.). - On $P L$ de Rham theory and rational homotopy type, Memoirs of AMS, 8, p. 179 (1976).

[6] Bousfield (A. K.), Kan (D. M.). - Homotopy limits, completions and localizations, Lect. Notes in Math. No.304, Springer-verlag, Berlin-Heidelberg-New York (1972).

[7] Brown (E.). - Twisted tensor products, Ann. of Math, 69, p. 223-246 (1959).

[8] Chen (K.T.). - Iterated integrals of differential forms and loop space homology, Ann. Math. 97, p. 217-246 (1973).

[9] Chen (K.T.). - Extension of $C^{\infty}$-function algebra by integrals and Malcev completion of $\pi_{1}$, Adv. Math. 23, p. 181-210 (1977).

[10] Dupont (J.-L.). - Simplicial de Rham cohomology and characteristic classes of flat bundles. Topology 15, p. 233-245 (1976)

[11] Getzler (E.), Jones (J.D.S.), Petrack (S.). - Differential forms on loop space and the cyclic bar complex, Topology 30, p. 339-371 (1991).

[12] Goerss (P.G.), Jardine (J.F.). - Simplicial homotopy theory, Birkhäuser (1997).

[13] Grothendieck (A.). - On the De Rham cohomology of algebraic varieties, Publ. math. de l'IHES, tome 29, p. 95-103 (1966).

[14] Jones (J.D.S.). - Cyclic homology and equivariant homology, Invent. Math. 87, p. 403-423 (1987).

[15] Kan (D. M.). - A combinatorial definition of homotopy groups, Ann. of Math. 67 , p. 282-312 (1958).

[16] McLane (S.). - Homology, Springer-Verlag, Berlin-Heidelberg-New York (1963).

[17] MAY (J.P.). — Simplicial methods in algebraic topology, U.Chicago (1967). 


\section{G. Sharygin}

[18] OnishChik (A.L.). - Topology of transitive transformation groups, Fizmatlit, Moscow (1995), in Russian

[19] Segal (G.). - Categories and cohomology theories, Topology 13, p. 293-312 (1974).

[20] Smirnov (V.A.). - Functor $D$ for twisted tensor products, Mat. Zametki 20, p. 465-472 (1976), in Russian.

[21] Smirnov (V.A.). - Functor $D$ and the strong homotopy, Mat. Zametki 21, p. 557-564 (1977), in Russian.

[22] Smirnov (V.A.). - Simplicial and Operad Methods in Algebraic Topology, AMS, Transl. Math. Monogr. 198 (2001).

[23] Sharygin (G.I.). - Local formulae for characteristic classes of a principal $G l_{n-}$ bundle, Matematicheskii Sbornik 199:10, p. 127-158 (2008), in Russian; translated in: Sbornik: Mathematics 199:10 p. 1547-1577 (2008).

[24] Sнiн (W.T.). - Homology des espaces fibrés, Publications Math. de l'IHES 13, p. 93-176 (1962).

[25] Sweedler (M.E.). — Hopf algebras, W. A. Benjamin, New-York (1969).

[26] Woronowicz (S.L.). - Differential calculus on compact matrix pseudogroups (Quantum Groups) Comm. Math. Phys. 122 Nr 1, p. 125-170 (1989).

[27] Zamboni (L.Q.). - A Chern character in cyclic homology, Trans. of AMS 331, p. $157-163$ (1992). 